\title{
On the Kodaira Dimension of the Moduli Space of Curves
}

\section{Citation}

Harris, Joe, and David B. Mumford. 1982. On the Kodaira dimension of the moduli space of curves. Inventiones Mathematicae 67(1): 23-86.

\section{Published Version}

doi:10.1007/BF01393371

\section{Permanent link}

http://nrs.harvard.edu/urn-3:HUL.InstRepos:3613574

\section{Terms of Use}

This article was downloaded from Harvard University's DASH repository, and is made available under the terms and conditions applicable to Other Posted Material, as set forth at http:// nrs.harvard.edu/urn-3:HUL.InstRepos:dash.current.terms-of-use\#LAA

\section{Share Your Story}

The Harvard community has made this article openly available.

Please share how this access benefits you. Submit a story.

\section{Accessibility}




\section{On the Kodaira Dimension of the Moduli Space of Curves}

Joe Harris and David Mumford

The purpose of this paper is to prove that the moduli space $\mathscr{M}_{g}$ of curves of genus $g$ over $\mathbf{C}$ is of general type if $g$ is odd and $g \geqq 25$. Moreover, the Kodaira dimension is at least 0 if $g=23$. It appears that a variant of our technique, which is technically more difficult, will prove that $\mathscr{M}_{g}$ is of general type for all sufficiently large $g$. In order to keep this paper to a reasonable length, we are treating only the odd genus case here, postponing the even genus case to a later paper.

The result of this paper should be contrasted with an earlier weaker result [12] to the effect that $\mathscr{M}_{g}$ is of "log general type" in the sense that there are enough $n$-canonical forms ${ }^{1} \omega$ on $\mathscr{M}_{\mathrm{g}}$-Sing $\left(\mathscr{M}_{\mathrm{g}}\right)$ with logarithmic poles at infinity (i.e., if $\overline{\mathscr{M}}_{g}-\mathscr{M}_{g}=V\left(x_{1}\right)$, then $\left.\omega=a(x)\left(d x_{1} \wedge \ldots \wedge d x_{3 g-3} / x_{1}\right)^{\otimes n}\right)$ to separate points generically. By the results of Brylinski [3], this implies that for all $g$, moduli spaces $\mathscr{M}_{\mathrm{g}}^{(\alpha)}$ of sufficiently high level $\alpha$ are of general type (the levels in question are non-abelian levels).

The proof is based on the analysis of a special divisor $D_{k} \subset \mathscr{M}_{g}$ with a very natural geometric meaning:

$$
D_{k}=\left(\text { locus of curves } C \text { which are } k \text {-fold covers of } \mathbf{P}^{1} \text { where } k=\frac{g+1}{2}\right) \text {. }
$$

We consider the closure $\bar{D}_{k}$ of $D_{k}$ in $\bar{M}_{g}$ and compute the divisor class of $\bar{D}_{k}$ in terms of the basic divisor classes

$$
\lambda, \delta_{0}, \delta_{1}, \ldots, \delta_{[\mathrm{g} / 2]} \in \operatorname{Pic}\left(\mathscr{M}_{\mathrm{g}}\right) \otimes \mathbf{Q}
$$

introduced in [13]. Here $\lambda$ is $c_{1}$ of the "Hodge bundle" $L_{\lambda}$, where $L_{\lambda}$ has fibre $A^{g} H^{0}\left(\omega_{\mathrm{C}}\right)$ over a curve $C$, and $\delta_{i}$ are the divisor classes of components $\Delta_{i}$ of $\overline{\mathscr{M}}_{\mathrm{g}}-\mathscr{M}_{\mathrm{g}}$. In particular, $\Delta_{0}$ is the closure of the locus of irreducible singular curves. The final result of $\S 5$ will be:

\footnotetext{
as

By an $n$-canonical form on a smooth variety $X^{r}$, we mean a differential form locally expressed

$$
a\left(x_{1}, \ldots, x_{r}\right)\left(d x_{1} \wedge \ldots \wedge d x_{r}\right)^{\otimes n}
$$
}




$$
\text { Class of } \bar{D}_{k} \equiv \frac{(2 k-4) !}{k !(k-2) !}\left\{6(k+1) \lambda-k \delta_{0}-\sum_{\alpha=1}^{k-1} 3 \alpha(2 k-1-\alpha) \delta_{\alpha}\right\} \text {. }
$$

On the other hand, using Grothendieck's Riemann-Roch formula, we calculate in $\S 2$ the canonical class of $\overline{\mathscr{M}}_{\mathrm{g}}$ :

$$
K_{\bar{M}_{\mathrm{g}}} \equiv 13 \lambda-2 \delta_{0}-3 \delta_{1}-2 \delta_{2}-\ldots-2 \delta_{\left[\frac{g}{2}\right]} .
$$

Putting these together proves

$$
c_{k} K_{\bar{M}_{\mathrm{g}}} \equiv 2 \bar{D}_{k}+c_{k}\left(1-\frac{12}{k}\right) \lambda+\left(\begin{array}{l}
\text { comb. of } \delta_{1}, \ldots, \delta_{\left[\frac{g}{2}\right]} \\
\text { with positive coefficients }
\end{array}\right)
$$

if $c_{k}=(2 k-4) ! /(k-1) !(k-2)$ !. It turns out also that the singularities of $\overline{\mathscr{M}}_{\mathrm{g}}$ are sufficiently mild so that all divisors in $\left|n K_{\bar{M}_{0}}\right|$ define $n$-canonical differential forms on $\overline{\mathscr{M}}_{\mathrm{g}}$ without poles on the resolution of $\overline{\mathscr{M}}_{\mathrm{g}}$. Thus if $|n K|_{\text {bir }}$ denotes the linear system of birationally holomorphic forms, we have

$$
\mid n c_{k} K_{\left.\bar{M}_{g}\right|_{\text {bir }}} \supset(\text { eff. div. })+\left|n c_{k}\left(1-\frac{12}{k}\right) \lambda\right| \text {. }
$$

Since $\lambda$ is ample on Satake's compactification $\overline{\mathscr{A}}_{g}$, the sections of $L_{\lambda}^{\otimes n}$ on $\overline{\mathscr{M}}_{g}$ define a birational morphism to $\mathbf{P}^{N}$. Therefore if $k=12,\left|c_{k} K_{\bar{M}_{\mathrm{g}}}\right|_{\mathrm{bir}} \neq \phi$, and if $k>12,\left|n c_{k} K_{\bar{M}_{g}}\right|_{\text {bir }}$ defines a birational map for $n \gg 0$.

The idea of this proof owes a great deal to $E$. Freitag and Y.-S. Tai, although most of the links to their work are not apparent. First and foremost, it was Freitag who saw beyond the classical picture according to which geometrically natural moduli spaces all seemed to be unirational, although their "higher level" variants were of general type: a picture largely based on the heavily studied case of $\mathscr{M}_{1}$, the moduli space of elliptic curves. Freitag showed that the moduli space $\mathscr{A}_{\mathrm{g}}$ of principally polarized abelian varieties is not unirational for $g \equiv 1(8), g \geqq 17^{g}$ [4], and for $24 \mid g$ [5]. Tai then showed that, in fact, $\mathscr{A}_{\mathrm{g}}$ is of general type for all $g \geqq 9$ ([17] to be published). He introduced 2 important new techniques: the first was the proof that the singularities of $\mathscr{A}_{\mathrm{g}}$ "didn't matter", except for the one along the $1^{\text {st }}$ boundary component $\mathscr{A}_{g-1}$. In particular, Tai and, independently, Reid [15] found an important hypothesis on the action of a finite group $G$ on $\mathbf{C}^{r}$ which implies that $n$-canonical differential forms on $\mathbf{C}^{r} / G$ have no poles on the resolution of $\mathbf{C}^{r} / G$. We use this criterion again here. Secondly, by means of the Hirzebruch Proportionality theorem, Tai proved that if $g$ is large enough, there are Siegel modular forms of weight $n(g)$, vanishing at $\mathscr{A}_{g-1}$ to order $m(g)$ with $m(g) / n(g)$ arbitrarily large. Subsequent to this, E. Freitag and one of the authors discussed at length how these techniques could be extended to $\mathscr{M}_{\mathrm{g}}$. They sought to use mixed holomorphic tensors on $\mathscr{A}_{g}$, i.e., differential forms which are not exterior forms, or symmetric forms, but sections of the full tensor power $\left(\Omega_{\mathscr{A}_{g}}^{1}\right)^{\otimes n}$, so as to produce from theta series $n$-canonical differential forms on $\mathscr{M}_{g}$. This still looks quite hopeful. But what led directly to the present paper was the discovery of a particular holomorphic tensor on $\mathscr{A}_{\mathrm{g}}$ which defines a map from the ring of 
Siegel modular forms vanishing at $\mathscr{A}_{g-1}$ to sufficiently large order to the pluricanonical ring of $\mathscr{M}_{g}$. (It is still not clear whether or not all Siegel modular forms with this vanishing at $\mathscr{A}_{g-1}$ do not, by some fluke, also vanish on the locus of Jacobians, in which case this map is zero.) But the forms defined by this map must at least vanish on a certain divisor $D^{\prime} \subset \mathscr{M}_{\mathrm{g}}$ :

$$
D^{\prime}=\left\{\begin{array}{l}
\text { locus of curves } C \text { with a line bundle } L \text { such that } \\
h^{0}(L) \geqq 2, h^{0}\left(\Omega_{\mathbf{C}}^{1} \otimes L^{-2}\right) \geqq 1
\end{array} .\right.
$$

If $g$ is odd, the $D$ above is one of the components of $D^{\prime}$. In this roundabout way, Freitag's techniques pointed directly to the method of the present proof!

This paper suggests that there is much interest in a systematic investigation of the relations between the fundamental classes of the many subvarieties of $\mathscr{M}_{\mathrm{g}}$ defined by geometric conditions, with the hope of establishing a calculus of such cycles. Such a study was in fact first undertaken by Steven Diaz, who is investigating the global geometry of the locus in $\overline{\mathscr{M}}_{\mathrm{g}}$ of curves with abnormal Weierstrass points; his work has been extremely valuable in developing the techniques of this paper. Hopefully, a more complete picture of the geometry of $\mathscr{M}_{\mathrm{g}}$ will emerge from such investigations; a great deal of work remains to be done. Another important question is whether $\mathscr{M}_{\mathrm{g}}$ actually carries non-zero holomorphic exterior $p$-forms for some $p$. The analogous theory for $\mathscr{A}_{\mathrm{g}}$ suggests that this might happen for $p=g, 2 g-1$ or $3 g-3$ (cf. Anderson [1], Stillman [16]).

The paper is organized as follows. In $\S 1$, we use the Reid-Tai criterion to prove that $n$-canonical forms on the open set $\overline{\mathscr{M}}_{\mathrm{g}}^{0}$ parametrizing curves without automorphisms automatically are holomorphic on a resolution of $\overline{\mathscr{M}}_{\mathrm{g}}$. In $\S 2$, we compute $K_{\bar{M}_{\mathrm{g}}}$. In $\S 3$ and $\S 6$, we calculate the class of $\bar{D}_{k}$, using a compactification of the Hurwitz moduli scheme of $k$-fold coverings, developed in $\S 4$, and using counts of the number of pencils of certain types on a generic curve of genus $g$, due to Griffiths and Harris, developed in $\S 5$.

In order to indicate the significance of this result we would like to point out the following easy result:

Proposition. Assume for some $g$ that the Kodaira dimension of $\mathscr{M}_{g}$ is at least 0 . Then if $C$ is a generic curve of genus $g$ (i.e. the corresponding point $[C] \in \mathscr{M}_{\mathrm{g}}$ lies in no subvariety defined over $\mathbf{Q}$ ), and $F$ is an algebraic surface containing $C$ on which $C$ moves in a non-trivial linear system, then $F$ is birational to $C \times \mathbf{P}^{1}$.

Proof. The Kodaira dimension being at least 0 means that $\mathscr{M}_{g}$ carries a $n$ canonical differential $a(x)\left(d x_{1} \wedge \ldots \wedge d x_{3 g-3}\right)^{\otimes n}$ with no poles on a compact smooth model of $\mathscr{M}_{g}$. This implies that $\mathscr{M}_{g}$ is not "uniruled", i.e. there is no dominant rational map

$$
\mathbf{P}^{1} \times W^{3 g-4} \rightarrow \mathscr{M}_{g}
$$

Therefore the images of all non-constant maps

$$
\mathbf{P}^{1} \rightarrow \mathscr{M}_{g}
$$


lie on subvarieties of $\mathscr{M}_{\mathrm{g}}$ defined over $\mathbf{Q}$. Therefore, given $C \in|C|$ on $F$, a pencil in $|C|$ defines a map from $\mathbf{P}^{1}$ to $\mathscr{M}_{g}$ through $[C]$, which must be constant, i.e. all $C^{\prime} \in|C|$ are isomorphic to $C$. Since $C$ has no automorphism, this means that $F$ is birational to $C \times \mathbf{P}^{1}$. Q.E.D.

Corollary. If $\mathrm{g}$ is odd, $\mathrm{g} \geqq 23$, then a generic curve of genus $\mathrm{g}$ does not occur in a non-trivial linear system on any non-ruled surface.

\section{§1. Pluri-canonical Forms on $\overline{\mathscr{M}}_{g}$}

If $V^{n}$ is any quasi-projective variety, by $k$-canonical forms on $V$ we understand holomorphic tensors $\omega$ given on the open set $V_{\text {reg }}$ of smooth points of $V$ by

$$
\omega=a\left(x_{1}, \ldots, x_{n}\right)\left(d x_{1} \wedge \ldots \wedge d x_{n}\right)^{\otimes k}
$$

such that for one and hence all desingularizations

$$
\pi: \tilde{V} \rightarrow V
$$

of $V, \omega$ extends to a holomorphic tensor of this type on all of $\tilde{V}$. Pluricanonical forms ${ }^{2}$ refers to $k$-canonical forms for all $k$. If $V$ is smooth, then $\sigma_{V}(n K)$ is the sheaf of $n$-canonical forms on $V$.

As in the introduction, $\overline{\mathscr{M}}_{\mathrm{g}}$ stands for the coarse moduli space of stable curves of genus $g$ : it is a normal projective variety. Related to $\overline{\mathscr{M}}_{g}$, we introduce the following further varieties:

$$
\begin{aligned}
& \qquad \overline{\mathscr{M}}_{g} \leftarrow \tilde{\mathscr{M}}_{g}=\text { desingularization of } \overline{\mathscr{M}}_{g} \\
& \begin{array}{l}
\text { open set of smooth } \\
\text { pts. of } \mathscr{M}_{g} \\
\text { open set of curves } C \\
\text { w/o automorphisms }
\end{array}=\overline{\mathscr{M}}_{g, \text { reg }}^{0}
\end{aligned}
$$

The purpose of this section is to prove the following theorem:

Theorem 1. If $\mathrm{g} \geqq 4$, then for all $n$, every $n$-canonical form on $\overline{\mathscr{M}}_{\mathrm{g}}^{0}$ extends to an $n$-canonical form on $\overline{\mathscr{M}}_{\mathrm{g}}$. More precisely:

$$
\Gamma\left(\overline{\mathscr{M}}_{\mathrm{g}}^{0}, \sigma_{\overline{\mathcal{M}}_{\mathrm{g}}}(n K)\right)=\Gamma\left(\tilde{\mathscr{M}}_{\mathrm{g}}, \sigma_{\tilde{\mathcal{M}}_{\mathrm{g}}}(n K)\right) .
$$

Proof. Recall that locally $\overline{\mathscr{M}}_{g}$ can be described as follows: let $C$ be a stable curve of genus $g$ and let

$$
\pi: \mathscr{C} \rightarrow \Delta^{3 g-3}
$$

2 There is a general confusion of terminology for referring to these and other tensor forms which are not exterior forms. We suggest $k$-canonical forms as a good phrase to distinguish these from exterior $k$-forms or symmetric $k$-forms 
be its local universal deformation space. $\operatorname{Aut}(C)$ is a finite group which operates on $\mathscr{C}$ and $\Delta^{3 g-3}$. Then a neighborhood of the point $[C] \in \overline{\mathscr{M}}_{\mathrm{g}}$ defined by $C$ is isomorphic as analytic space to

$$
\Delta^{3 g-3} / \operatorname{Aut}(C)
$$

Moreover the action of a finite group on a smooth space can always be made linear in suitable coordinates, hence if $T_{0, \Delta}$ is the tangent space to $\Delta^{3 g-3}$ at 0 ,

$$
\Delta^{3 g-3} / \operatorname{Aut}(C) \cong \text { neigh. of } 0 \text { in }\left[T_{0, \Delta} / \operatorname{Aut}(C)\right] \text {. }
$$

On the other hand, $T_{0, \Delta}$ is the space of infinitesimal deformations of $C$ which is well known to be

$$
\operatorname{Ext}^{1}\left(\Omega_{C}^{1}, o_{C}\right)
$$

where $\Omega_{C}^{1}$ is the sheaf of Kähler differentials on $C$. By Serre duality,

$$
\operatorname{Ext}^{1}\left(\Omega_{C}^{1}, \sigma_{C}\right) \cong H^{0}\left(\Omega_{C}^{1} \otimes \omega_{C}\right)^{*}
$$

where $\omega_{C}$ is the dualizing sheaf on $C^{3}$. Thus finally

(neigh. of $[C]$ in $\left.\overline{\mathscr{M}}_{\mathrm{g}}\right) \cong\left(\right.$ neigh. of 0 in $H^{0}\left(\Omega_{C}^{1} \otimes \omega_{C}\right)^{*} / \operatorname{Aut}(C)$ ).

Note that in this description, the open set of points on the left which are in $\mathscr{M}_{\mathrm{g}}^{0}$ is equal to the open set of points on the right where $\operatorname{Aut}(C)$ acts freely.

We have therefore particular cases of the problem:

$V$ a vector space of dimension $d$

$G \subset G L(V)$ a finite group

$V_{0} \subset V$ open set where $G$ acts freely

When do $n$-canonical forms on $V_{0} / G$ extend holomorphically to a resolution $V / G$ of $V / G$ ? [17]:

The following criterion is due independently to M. Reid [15] and Y.-S. Tai

Reid-Tai Criterion: In the above situation, for all $g \in G$, let $g$ be conjugate to

$$
\left(\begin{array}{cccc}
a_{1} & & & \\
\zeta & \ddots & & 0 \\
& & \ddots & \ddots \\
& 0 & & a_{d} \\
& & & \zeta
\end{array}\right)
$$

where $\zeta$ is a primitive $m^{\text {th }}$ root of 1 , and $0 \leqq a_{i}<m$. If for all $g$ and $\zeta$,

3 If $C$ is smooth, $\omega_{C} \cong \Omega_{C}^{1}$. But at double points given by $x y=0, \Omega_{C}^{1}$ is generated by $d x, d y \bmod$ one relation $x d y+y d x=0$, and has the torsion submodule $\mathbf{C} \cdot(x d y)$, while $\omega_{C}$ is free on one generator given by the differentials $\frac{d x}{x}$ on $y=0,-\frac{d y}{y}$ on $x=0$ 


$$
\sum_{i=1}^{d} a_{i} / m \geqq 1
$$

then any $n$-canonical form on $V_{0} / G$ extends holomorphically to $V / G$.

In fact, we need a slight generalization of the Reid-Tai criterion. This says that for any $G$-action, certain $n$-canonical forms $\omega$ extend to $V / G$, namely those which are holomorphic on $V_{0} / G$ and, for all $g \in G$ for which $\sum a_{i} / m<1$ (for some $\zeta$ ) - call these bad $g$ 's - $\omega$ is also holomorphic on the divisors $E \subset V / G$ mapping onto

$$
\operatorname{Im}(\{x \in V \mid g x=x\} \rightarrow V / G) .
$$

Equivalently, we must assume $\omega$ to be holomorphic on the resolution of some open set $U \subset V / G$, where $U$ contains $V_{0} / G$ and $U$ contains the image in $V / G$ of the generic point $\eta_{g}$ of $\{x \in V \mid g x=x\}$, for all bad $g$. We will give a proof in appendix 1 to this section.

Our main task is therefore to investigate, case-by-case, what are the eigenvalues of an automorphism $\phi$ of a stable curve $C$ acting on $H^{0}\left(\Omega_{C}^{1} \otimes \omega_{C}\right)$. We take the case of a smooth $C$ first.

Proposition. Let $C$ be a smooth curve over $\mathbf{C}$, and let $\phi$ be an automorphism of $C$ of order $n$. Let $\zeta$ be any primitive $n^{\text {th }}$ root of 1 , and let the action $\phi$ on $H^{0}\left({ }_{0} C(2 K)\right) *$ be given by

$$
\left(\begin{array}{cccc}
a_{1} & & \\
\zeta & \ddots & & 0 \\
& \ddots & \ddots & a_{3 g-3} \\
0 & & \zeta^{3}
\end{array}\right) \text { where } 0 \leqq a_{i}<n
$$

Then either

$$
\sum_{i=1}^{3 g-3}\left(a_{i} / n\right) \geqq 1
$$

or else $(C, \phi)$ is one of the following cases:

i) genus $C=0$

ii) genus $C=1$

iii) genus $C=2, n=2, \phi$ is the hyperelliptic involution

iv) genus $C=2, C$ is a double cover of an elliptic curve and $\phi$ is the associated involution

v) genus $C=3, C$ is hyperelliptic and $\phi$ is the hyperelliptic involution.

Proof. Let $C_{0}$ be the quotient of $C$ by $\phi$, let $g_{0}$ be the genus of $C_{0}$ and let $P_{1}, \ldots, P_{\beta} \in C_{0}$ be the branch points of the cover

$$
\pi: C \rightarrow C_{0} .
$$

Let $m_{i}=$ order of branching of $\pi$ over $P_{i}$ : i.e., there are $n / m_{i}$ points $Q_{i, \alpha} \in C$ over $P_{i}$ and $\pi$ is given locally at each $Q_{i, \alpha}$ by the $m_{i}^{\text {th }}$ root of a suitable local parameter at $P_{i}$. 
Consider the family of cyclic branched coverings

$$
\pi^{\prime}: C^{\prime} \rightarrow C_{0}^{\prime}
$$

which you get by varying the moduli of $C_{0}$ and varying the branch points $P_{i} \in C_{0}$. The dimension of this family is $3 g_{0}-3+\beta$. This defines a subvariety $W \subset \mathscr{M}_{g}$ of codimension $3\left(g-g_{0}\right)-\beta$ containing $C$ such that all $C^{\prime} \in W$ admit automorphisms $\phi^{\prime}$ deforming $\phi$. On the other hand, if the local deformation space of $C$ is $p: \mathscr{C} \rightarrow S, S$ a germ of smooth $(3 g-3)$-dimensional manifold, then $\phi$ acts naturally on $\mathscr{C}$ and $S$ :

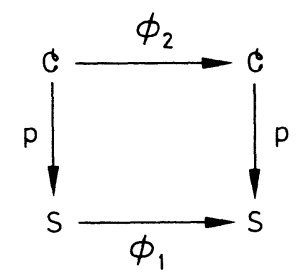

In suitable coordinates on $S, \phi_{1}$ acts linearly. The subvariety $W$ of $\mathscr{M}_{g}$ is just the image of the fixed point set of $\phi_{1}$ on $S$. Therefore, the dimension of $W$ is the dimension of the subspace of $T_{0, S} \cong H^{0}\left(o_{C}(2 K)\right)$ fixed by $\phi$, i.e., the number of $a_{i}$ equal to 0 . This proves:

$$
\begin{aligned}
& \#\left\{i \mid a_{i}=0\right\}=3 g_{0}-3+\beta, \\
& \#\left\{i \mid a_{i} \geqq 1\right\}=3\left(g-g_{0}\right)-\beta .
\end{aligned}
$$

Therefore

$$
\sum_{i=1}^{3 g-3}\left(a_{i} / n\right) \geqq \frac{3\left(g-g_{0}\right)-\beta}{n} .
$$

But by Hurwitz's formula:

$$
2 g-2=n\left(2 g_{0}-2\right)+\sum_{i=1}^{\beta} \frac{n}{m_{i}}\left(m_{i}-1\right) .
$$

Let's now assume $\sum\left(a_{i} / n\right)<1$, hence in particular

$$
n>3\left(g-g_{0}\right)-\beta .
$$

Combining this with Hurwitz's formula, you easily check:

$$
\frac{2}{3}>\frac{2 n-2}{n}\left(g_{0}-1\right)+\sum_{i=1}^{\beta}\left(1-\frac{2}{3 n}-\frac{1}{m_{i}}\right) \text {. }
$$

Note that as $n \geqq 2, m_{i} \geqq 2$,

$$
1-\frac{2}{3 n}-\frac{1}{m_{i}} \geqq \frac{1}{6}>0,
$$

hence $(*)$ implies immediately that $g_{0} \leqq 1$.

Moreover, the $m_{i}$ cannot be chosen as arbitrary divisors of $n$ because of the following: 
Lemma. Let $M=$ l.c.m. $\left(m_{i}\right)$. For all primes $p$ dividing $M$, let $p^{r} \mid M, p^{r+1} \nmid M$, and let

$$
I=\left\{i\left|p^{r}\right| m_{i}\right\} .
$$

Then I has at least 2 elements in it, and if $p=2, I$ consists of an even number of i's. Moreover, if $g_{0}=0, n=M$.

Proof. The covering $C$ of $C_{0}$ is defined by a surjective homomorphism:

$$
\pi_{1}\left(C_{0}-\left\{P_{1}, \ldots, P_{\beta}\right\}\right) \rightarrow \mathbf{Z} / n \mathbf{Z} .
$$

This factors through $H_{1}\left(C_{0}-\left\{P_{1}, \ldots, P_{\beta}\right\}\right)$ because $\mathbf{Z} / n \mathbf{Z}$ is abelian. Let $e_{i}$ be a small loop around $P_{i}$. Then $H_{1}\left(C_{0}-\left\{P_{1}, \ldots, P_{\beta}\right\}\right)$ is the direct sum of $H_{1}\left(C_{0}\right)$ and $\oplus \mathbf{Z} e_{i}$ modulo the one relation $\sum e_{i}=0$. Let $e_{i}$ be mapped to $\bar{e}_{i} \in \mathbf{Z} / n \mathbf{Z}$. It's easy to see that $m_{i}$ is exactly the order of $\bar{e}_{i}$. But

a) $\sum_{i=1} \bar{e}_{i}=0$ in $\mathbf{Z} / n \mathbf{Z}$

and

b) if $g_{0}=0$, hence $H_{1}\left(C_{0}\right)=(0),\left\{\bar{e}_{i}\right\}$ generate $\mathbf{Z} / n \mathbf{Z}$.

With the notation in the lemma, if $m=M / p$

$$
\sum_{i=1}^{\beta} m \bar{e}_{i}=0 \text { and } m \bar{e}_{i}=0 \text { if } i \notin I .
$$

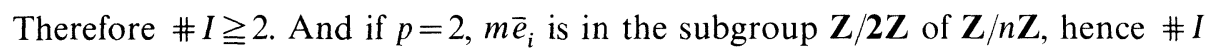
is even. Also, if $g_{0}=0$, then $M=n$ by (b).

In particular, this shows that $\beta=0$ or $\beta \geqq 2$, and if $n=2$, then $\beta$ is even. Using these restrictions on the $m_{i}$, a lengthy but straightforward calculation allows one to list all the solutions to (*). The result is:

a) $g_{0}=1, \beta=0$

b) $g_{0}=1, n=\beta=2$, all $m_{i}=2$

c) $g_{0}=0, n=2, \beta=2,4,6$ or 8 , all $m_{i}=2$

d) $g_{0}=0, \beta=2$, any $n$

e) $g_{0}=0, \beta=3$ with one of the following triples $\left(m_{1}, m_{2}, m_{3}\right)$ :

$$
\begin{array}{ll}
(2,4 k, 4 k) & n=4 k \\
(2,2 k, k), k \text { odd } & n=2 k \\
(3,3 k, 3 k) & n=3 k \\
(3,3 k, k), 3 \nmid k & n=3 k
\end{array}
$$

$(4,4 k, k), k$ odd, $5 \leqq k \leqq 13$

$(4,4 k, 2 k), k=3,5,7$

$(4,8 k, 8 k), k=1,2$

$(5,5 k, 5 k), k=1,2$

$(5,5 k, k), k=6,7,8$

$(7,7,7)$ 
f) $g_{0}=0, \beta=4$ with one of the following quadruples $\left(m_{1}, m_{2}, m_{3}, m_{4}\right)$ :

$$
\begin{array}{lll}
(2,2, k, k), & k=3,5, & n=2 k \\
(2,2,2 k, 2 k), & k=2,3, & n=2 k \\
(3,3,3,3), & & n=3 \\
(2,6,3,3), & & n=6 .
\end{array}
$$

The Proposition asserts that a), b), c) and d) are in fact the only cases where $\sum a_{i} / n<1$. In cases e) and f) we need to look more closely and evaluate the $a_{i}$ s.

For (f), we can describe the curves $C$ which occur as follows:

for $(2,2, k, k), k$ odd, $C$ and $\phi$ are

$$
\begin{aligned}
y^{2} & =\left(x^{k}-1\right)\left(x^{k}-a\right) \\
\phi(x, y) & =\left(\zeta_{k} x,-y\right), \zeta_{k} \text { a primitive } k^{\text {th }} \text { root of } 1
\end{aligned}
$$

for $(2,2,2 k, 2 k), C$ and $\phi$ are

$$
\begin{aligned}
y^{2} & =x\left(x^{k}-1\right)\left(x^{k}-a\right) \\
\phi(x, y) & =\left(\zeta_{k} x, \zeta_{2 k} y\right), \zeta_{2 k}^{2}=\zeta_{k}
\end{aligned}
$$

for $(3,3,3,3), C$ is

$$
y^{2}=\left(x^{3}-1\right)\left(x^{3}-a\right) \text { again }
$$

but $\phi$ is

$$
\phi(x, y)=\left(\zeta_{3} x, y\right), \zeta_{3} \text { a primitive } 3^{\text {rd }} \text { root of } 1,
$$

for $(2,6,3,3), C$ is the non-hyperelliptic curve of genus 3

$$
\begin{aligned}
y^{3} & =\left(x^{2}-1\right)\left(x^{2}-a\right) \\
\phi(x, y) & =\left(-x, \zeta_{3} y\right) .
\end{aligned}
$$

From this description, one calculates the $a_{i}$ 's in this table:

\begin{tabular}{l|l}
$\left(m_{1}, m_{2}, m_{3}, m_{4}\right)$ & $\left(a_{i} / n\right)$ \\
\hline$(2,2,3,3)$ & $(0 / 6,2 / 6,4 / 6)$ \\
$(2,2,4,4)$ & $(0 / 4,2 / 4,2 / 4)$ \\
$(2,2,5,5)$ & $\left(\frac{0}{10}, \frac{1}{10}, \frac{2}{10}, \frac{4}{10}, \frac{4}{10}, \frac{6}{10}, \frac{6}{10}, \frac{8}{10}, \frac{9}{10}\right)$ \\
$(2,2,6,6)$ & $\left(\frac{0}{6}, \frac{2}{6}, \frac{2}{6}, \frac{3}{6}, \frac{4}{6}, \frac{4}{6}\right)$ \\
$(3,3,3,3)$ & $(0 / 3,1 / 3,2 / 3)$ \\
$(2,6,3,3)$ & $(0 / 6,1 / 6,2 / 6,3 / 6,4 / 6,4 / 6)$
\end{tabular}

which confirms the Proposition in this case. (Note that the $\zeta_{k}$ and $\zeta_{3}$ used to describe $\phi$ may be any primitive $k^{\text {th }}$ or $3^{\text {rd }}$ root of 1 , hence one must not merely check

$$
\sum a_{i} / n \geqq 1
$$


but also check that for all $j \in\{1, \ldots, n-1\}$ relatively prime to $n$,

$$
\sum \operatorname{res}_{n}\left(j \cdot a_{i}\right) / n \geqq 1
$$

where $\operatorname{res}_{n}(k)$ is the residue in $\{0,1, \ldots, n-1\}$ of $k \bmod n$.)

For (e), a similar check could be carried out, but because of its tediousness and the possibility of error, it seemed easier and much more convincing to write a computer program to calculate $\sum a_{i} / n$ one at a time for all cyclic covers of $\mathbf{P}^{1}$ with 3 branch points. This is reproduced in appendix 2 where a table of the minimum values of $\sum\left(a_{i} / n\right)$ for each $g \leqq 18$ is also given. On the other hand, the computer only checks finitely many cases and the $1^{\text {st }} 4$ types in (e) are infinite families. These are the curves

$$
\begin{array}{ll}
y^{2}=x^{k}-1, & \phi(x, y)=\left(\zeta_{k} x,-y\right), k \text { odd } \\
y^{2}=x\left(x^{2 k}-1\right), & \phi(x, y)=\left(\zeta_{2 k} x, \zeta_{4 k} y\right) \\
y^{3}=x\left(x^{k}-1\right), & \phi(x, y)=\left(\zeta_{k} x, \zeta_{3 k} y\right) \\
y^{3}=x^{2}\left(x^{k}-1\right), & \phi(x, y)=\left(\zeta_{k} x, \zeta_{3 k}^{2} y\right)
\end{array}
$$

for which the $a_{i}$ are readily worked out explicitly. We omit this.

Using this analysis as a building block, we look next at automorphism of singular stable curves:

Theorem 2. Let $C$ be a stable (possibly singular) curve of arithmetic genus $g$ and let $\phi$ be an automorphism of $C$ of order $n$. Let $\zeta$ be any primitive $n^{\text {th }}$ root of 1 and let the action of $\phi$ on $H^{0}\left(\Omega_{C}^{1} \otimes \omega_{C}\right)$ be given by

$$
\left(\begin{array}{cccc}
\zeta^{a_{1}} & & & 0 \\
& \ddots & \ddots & \\
& & \ddots & \\
& 0 & & \zeta^{a_{g-3}}
\end{array}\right) \text {, where } 0 \leqq a_{i}<n \text {. }
$$

We assume $\mathrm{g} \geqq 4$.

Then either

$$
\sum_{i=1}^{3 g-3} a_{i} / n \geqq 1
$$

or else $(C, \phi)$ belongs to one of the following cases:

(i) $C=C_{1} \cup C_{2}$ where genus $\left(C_{1}\right)=g-1$,

$C_{2}$ is either elliptic or rational with one node, $C_{1} \cap C_{2}=\{P\}, n=2$ and

$$
\begin{aligned}
& \left.\phi\right|_{C_{1}}=\text { identity } \\
& \left.\phi\right|_{C_{2}}=\text { inverse with respect to origin } P
\end{aligned}
$$

(ii) $C=C_{1} \cup C_{2}$ as above, but $C_{2}$ is elliptic with $j\left(C_{2}\right)=0, n=6$ and

$$
\begin{aligned}
& \left.\phi\right|_{C_{1}}=\text { identity } \\
& \left.\phi\right|_{C_{2}}=\text { one of the two automorphisms of } C_{2} \text { of order } 6 \text { fixing } P .
\end{aligned}
$$


(iii) $C=C_{1} \cup C_{2}$ as above, but $C_{2}$ is elliptic with $j\left(C_{2}\right)=12^{3}, n=4$ and

$$
\begin{aligned}
& \left.\phi\right|_{C_{1}}=\text { identity } \\
& \left.\phi\right|_{C_{2}}=\text { one of the two automorphisms of } C_{2} \text { of order } 4 \text { fixing } P .
\end{aligned}
$$

Proof. Note that we have proved the theorem for smooth curves $C$. Also note that the set of exceptions (i), (ii) and (iii) forms a closed set in the moduli space $\overline{\mathscr{M}}_{g}$. Therefore we can prove the theorem by induction on the number of double points $P \in C$, checking for each $(C, \phi)$ that either

a) $\sum\left(a_{i} / n\right) \geqq 1$ or

b) $(C, \phi)$ has a deformation $\left(C^{\prime}, \phi^{\prime}\right)$ with fewer double points.

This is because in any family $\mathscr{C} \rightarrow S$ with connected base and with an automorphism $\phi: \mathscr{C} \rightarrow \mathscr{C}$ over $S$ of order $n$, the eigenvalues of $\phi$ on $H^{0}\left(\Omega_{C_{s}}^{1}\right.$ $\left.\otimes \omega_{C_{s}}\right)$ vary continuously and are $n^{\text {th }}$ roots of 1 , hence are constant.

Note next $\Omega_{C}^{1} \otimes \omega_{C}$ has the following local description: at smooth points $P \in C$ with local coordinate $x$, it is an invertible sheaf with generator $d x^{\otimes 2}$; at ordinary double points $P \in C$, if $C$ is given locally by $x \cdot y=0$, it is generated by the differentials:

subject to the relation

$$
\begin{aligned}
& \omega_{1}=d x^{\otimes 2} / x, \\
& \omega_{2}=d y^{\otimes 2} / y
\end{aligned}
$$

$$
y \omega_{1}=x \omega_{2}
$$

Thus

$$
y \omega_{1}=\frac{y d x^{\otimes 2}}{x}=\frac{x d y^{\otimes 2}}{y}
$$

generates a submodule of dimension 1 over $k$ (because $x\left(y \omega_{1}\right)=0$ and $y\left(y \omega_{1}\right)$ $=y\left(x \omega_{2}\right)=0$ ), and mod this we have the direct sum of the sheaves of quadratic differentials on the 2 branches at $P$, with simple poles at $P$. This gives rise to the exact sequence:

$$
0 \rightarrow \underset{\substack{\text { doublepts. } \\ P \in C}}{\bigoplus}\left(\text { tor }_{P}\right) \rightarrow \Omega_{C}^{1} \otimes \omega_{C} \rightarrow \bigoplus_{\alpha} o_{C_{x}}\left(2 K_{C_{\alpha}}+\sum P_{\beta}\right) \rightarrow 0
$$

where $C_{\alpha}$ are the normalizations of the components of $C$, and for each $\alpha$, the $P_{\beta} \in C_{\alpha}$ are those points of $C_{\alpha}$ whose image in $C$ are double points of $C$. Therefore:

$$
0 \rightarrow \underset{\substack{\text { double } \\ \text { pts. } P}}{\oplus}\left(\operatorname{tor}_{P}\right) \rightarrow H^{0}\left(\Omega_{C}^{1} \otimes \omega_{C}\right) \rightarrow \bigoplus_{\alpha} H^{0}\left(o_{C_{\alpha}}\left(2 K_{\alpha}+\sum_{\beta} P_{\beta}\right)\right) \rightarrow 0
$$

The first step is to analyze the eigenvalues of $\phi$ on $\oplus\left(\right.$ tor $\left._{P}\right)$. Say $P$ is a double point of $C$ and $\left\{P, \phi P, \phi^{2} P, \ldots, \phi^{m-1} P\right\}$ are distinct, $\phi^{m} P=P$, where $m \mid n$. Then $\phi^{m}$ acts on $\operatorname{tor}_{P}$ and if this action is trivial, take $\bar{e} \in\left(\right.$ tor $\left._{P}\right)$ and consider the element $e \in H^{0}\left(\Omega_{C}^{1} \otimes \omega_{C}\right)$ given by

$$
e \in \underset{Q}{\oplus}\left(\operatorname{tor}_{Q}\right), \quad e_{Q}= \begin{cases}\phi^{i} \bar{e} & \text { if } Q=\phi^{i} P \\ 0 & \text { if } Q \notin\left\{P, \phi P, \ldots, \phi^{n-1} P\right\} .\end{cases}
$$


Then $\phi e=e$. Thus dually, $e$ defines a deformation of $C$ to which $\phi$ lifts and in which the double points $\phi^{i} P$ disappear. By induction on the number of double points, this case is taken care of. Next suppose $\phi^{m}$ acts non-trivially on (tor ${ }_{P}$ ). We then calculate all the eigenvalues of $\phi$ on

$$
\left(\text { tor }_{P}\right) \oplus \ldots \oplus\left(\text { tor }_{\phi^{m-1} P}\right) \text {. }
$$

Let $\zeta$ be a primitive $n^{\text {th }}$ root of 1 . We must have

Let

$$
\phi^{m} \bar{e}=\zeta^{m \ell} \bar{e}, \quad 1 \leqq \ell<\frac{n}{m} .
$$

Then

$$
e_{a}=\sum_{i=0}^{m-1} \zeta^{i a} \cdot\left(\phi^{i} \vec{e}\right)
$$

$$
\begin{aligned}
\phi\left(e_{a}\right) & =\sum_{i=0}^{m-1} \zeta^{i a} \cdot\left(\phi^{i+1} \bar{e}\right) \\
& =\sum_{i=1}^{m} \zeta^{(i-1) a}\left(\phi^{i} \bar{e}\right) \\
& =\zeta^{-a} \sum_{i=1}^{m-1} \zeta^{i a}\left(\phi^{i} \bar{e}\right)+\zeta^{(m-1) a} \cdot \zeta^{m \ell} \cdot \bar{e} \\
& =\zeta^{-a} \cdot e_{a}
\end{aligned}
$$

provided $m(a+l) \equiv 0(\bmod n)$, i.e., $a \equiv-l(\bmod n / m)$. Therefore

$$
\zeta^{\ell}, \zeta^{\ell+\frac{n}{m}}, \ldots, \zeta^{\ell+(m-1) \frac{n}{m}}
$$

are the eigenvalues of $\phi$ on

$$
\left(\operatorname{tor}_{P}\right) \oplus \ldots \oplus\left(\text { tor }_{\phi^{m-1} P}\right) .
$$

The corresponding part of the sum $\sum a_{i} / n$ works out to be

$$
\frac{\ell \cdot m}{n}+\frac{1}{m}(1+2+\ldots+(m-1))=\ell \cdot \frac{m}{n}+\frac{m-1}{2} .
$$

In particular, if $m \geqq 3$, it follows that $\sum a_{i} / n \geqq 1$ already. If $\sum\left(a_{i} / n\right)<1$, it follows that either

a) $\phi$ fixes all double points $P \in C$ or

b) $\phi$ fixes all but one pair $\{P, \phi P\}$ which are interchanged, and $n \geqq 6$. least

Moreover, if $C$ has $\delta$ double points, the torsion eigenvalues contribute at

$$
\delta / n, \quad \text { resp. } \delta / n+1 / 2
$$

to $\sum a_{i} / n$, in case a), resp. b).

The second step is to analyze the action of $\phi$ on the set of components of $C$. We shall prove that if for any $\alpha, \phi C_{\alpha} \neq C_{\alpha}$ then either $\sum a_{i} / n \geqq 1$ or $(C, \phi)$ 
has a deformation with fewer double points. To see this, consider that the image $C_{\alpha}^{\prime}$ of $C_{\alpha}$ in $C$ looks like:

(a) $C_{\alpha}=\mathbf{P}^{1}$,

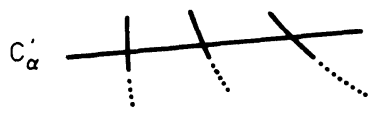

(b) $C_{\alpha} \cong \mathbf{P}^{1}$,

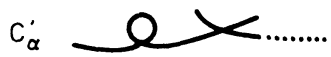

(c) $C_{\alpha} \cong \mathbf{P}^{1}$,

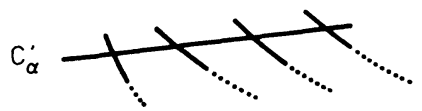

(d) $C_{\alpha} \cong \mathbf{P}^{1}$

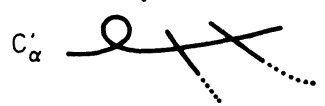

(e) $C_{\alpha}$ elliptic, $C_{\alpha}^{\prime}$

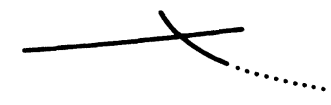
then

(f) If $g_{\alpha}=$ genus $\left(C_{\alpha}\right)$, and $\delta_{\alpha}=\#\left\{\right.$ pts. $P_{\beta} \in C_{\alpha}$ mapping to double pts. of $\left.C\right\}$,

$$
3 g_{\alpha}-3+\delta_{\alpha} \geqq 2 \text {. }
$$

As in the analysis of double points, suppose $C_{\alpha}, \phi C_{\alpha}, \ldots, \phi^{m-1} C_{\alpha}$ are distinct and $\phi^{m} C_{\alpha}=C_{\alpha}$. Look at the action of $\phi$ on

$$
W=\bigoplus_{k=0}^{m-1} H^{0}\left(\sigma_{\phi^{k} C_{\alpha}}\left(2 K_{\left(\phi^{k} C_{\alpha}\right)}+\sum P_{\beta}\right)\right) \text {. }
$$

The same calculation given for double points shows that if

$$
k=\operatorname{dim} H^{0}\left({ }^{\sigma_{\alpha}}\left(2 K_{\alpha}+\sum P_{\beta}\right)\right)
$$

and if the eigenvalues of $\phi^{m}$ here are $\zeta^{m \ell_{1}}, \ldots, \zeta^{m \ell_{k}}$, then the eigenvalues of $\phi$ on $W$ are

$$
\zeta^{\ell+j}\left(\frac{n}{m}\right), \quad 1 \leqq i \leqq k, \quad 0 \leqq j<\frac{n}{m},
$$

hence $W$ gives $a$ contribution to $\sum\left(a_{i} / n\right)$ at least equal to

$$
k \cdot \frac{m-1}{2} \text {. }
$$

Therefore if $\sum a_{i} / n<1$, either $k=0$ or $k=1$ and $m=2$. In cases a), b), $k=0$, in cases c), d), e), $k=1$ and in case f), $k \geqq 2$. On the other hand, in cases c), d), e), if any double point moves, it also gives a contribution of $1 / 2$ to $\sum a_{i} / n$, hence together with the contribution from $W, \sum a_{i} / n \geqq 1$. But if all double points are fixed, $\phi\left(C_{\alpha}^{\prime}\right)$ must be the second component through all double points $P$ where $C_{\alpha}^{\prime}$ meets another component of $C$, i.e., $C=C_{\alpha}^{\prime} \cup \phi\left(C_{\alpha}^{\prime}\right)$. In this case, one sees immediately that $g \leqq 3$. Turning to case a), at least one of the three double points on $C_{\alpha}^{\prime}$ must be fixed, call it $P_{0}$, hence $\phi\left(C_{\alpha}\right)$ must be the second component of $C$ through $P_{0}$. Therefore $\phi$ interchanges these 2 components and $\phi^{2}$ fixes $C_{\alpha}$. But $\phi^{2}$ also fixes all double points, hence $\phi^{2}$ is an automorphism 
of $C_{\alpha}^{\prime} \cong \mathbf{P}^{1}$ with 3 fixed points, hence $\left.\phi^{2}\right|_{c_{\alpha}}=$ identity. At $P_{0}$, let $x \cdot y=0$ be a local equation of $C$. Then $\left.\phi^{2}\right|_{C_{\alpha}}=$ identity implies that $\phi$ acts by:

$$
\begin{aligned}
& \phi^{*}(x)=y, \\
& \phi^{*}(y)=x .
\end{aligned}
$$

But then $\phi$ fixes the torsion differential $y d x^{\otimes 2} / x$ (or alternately, one can argue that $\phi$ lifts to the universal deformation $x y=t$ of $P_{0}$ by $\phi^{*} x=y, \phi^{*} y=x, \phi^{*} t$ $=t)$. Therefore $(C, \phi)$ has a deformation in which $P_{0}$ disappears. Finally, in case (b), the double point of $C_{\alpha}^{\prime}$ must move, hence the other double point of $C$ on $C_{\alpha}^{\prime}$ does not move, and the whole of $C$ will be nothing but $C_{\alpha}^{\prime} \cup \phi\left(C_{\alpha}^{\prime}\right)$. Then $g$ $=2$ which was excluded.

This reduces us to the case where $\phi$ fixes every component of $C$. But then every component $C_{\alpha}$ contributes to $\sum\left(a_{i} / n\right)$ the eigenvalues of $\phi$ on

$$
H^{0}\left({ }^{\circ} C_{\alpha}\left(2 K_{C_{\alpha}}+\sum P_{\beta}\right)\right) \text {. }
$$

In most cases, by the Proposition, the eigenvalues of $\phi$ on $H^{0}\left({ }^{\circ} C_{\alpha}\left(2 K_{C_{\alpha}}\right)\right)$ already give us $\sum\left(a_{i} / n\right) \geqq 1$. In particular, it follows that for all $\alpha$, one of the following is true:

a) $\left.\phi\right|_{C_{\alpha}}$ is the identity,

b) $C_{\alpha} \cong \mathbf{P}^{1}$,

c) $C_{\alpha}$ is elliptic,

d) $C_{\alpha}$ is hyperelliptic of genus 2 or $3,\left.\phi\right|_{C_{\alpha}}=$ hyperelliptic involution or

e) $C_{\alpha}$ has genus 2 , and is a double cover of an elliptic curve with $\left.\phi\right|_{C_{\alpha}}$ $=$ sheet interchange.

We can now argue that $\phi$ fixes all double points of $C$ too. This goes as follows: note that $\phi^{2}$ fixes all double points of $C$, hence $\phi^{4}$ fixes all pairs consisting of a double point of $C$ and a branch of $C$ at $P$, i.e., all $P_{\beta}$ in all $C_{\alpha}$. Since in case (b), $\mathbf{P}^{1}$ has at least three $P_{\beta}$ 's on it, $\left.\phi^{4}\right|_{C_{\alpha}}=$ identity in this case. Moreover in case (c), $\left.\phi\right|_{C_{\alpha}}$ either has a fixed point, hence has order $\leqq 6$, or is a translation. And if it is a translation, then $\left.\phi^{4}\right|_{C_{\alpha}}$ is still a translation and also fixes the points $P_{\beta}$ on $C_{\alpha}$, i.e., $\left.\phi^{4}\right|_{C_{\alpha}}=$ identity. Therefore in all cases the order of $\phi$ on each $C_{\alpha}$ is $1,2,3,4$ or 6 . Now suppose $\phi$ moves a double point. This can happen in 2 ways:

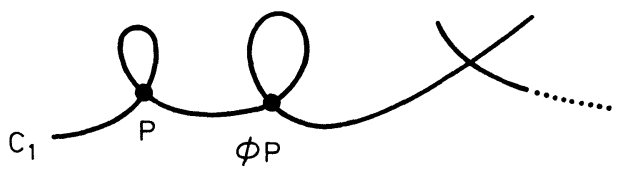

or

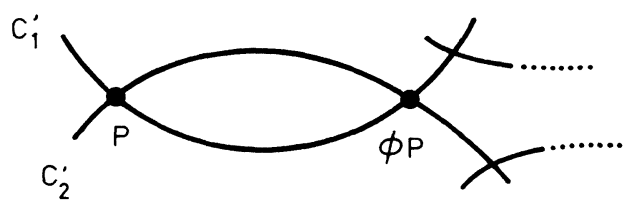


In the first case, tor $_{P} \oplus$ tor $_{\phi P}$ contributes at least $1 / 2+2 / n_{1}$ to $\sum a_{i} / n$ where $n_{1}$ $=$ order of $\left.\phi\right|_{C_{1}}$. If $n_{1} \leqq 4$, we are done. If $n_{1}=6$, then $C_{1}$ is elliptic with $j\left(C_{1}\right)$ $=0$. Then we get an extra term from the action of $\phi$ on

$$
H^{0}\left(o_{C_{1}}\left(2 K_{C_{1}}\right)\right) \subset H^{0}\left(o_{C_{1}}\left(2 K_{C_{1}}+\sum P_{\beta}\right)\right) \text {. }
$$

If $d z$ is the translation-invariant differential on $C_{1}$, then $d z^{\otimes 2}$ generates $\circ\left(2 K_{C_{1}}\right)$ and $\phi$ acts by $z_{1} \mapsto \zeta_{6} z_{1}$. Thus we have a term of $1 / 3$ and

$$
1 / 2+2 / 6+1 / 3>1
$$

In the second case, if $n_{1}=l \mathrm{~cm}$ (order of $\phi$ on $C_{1}, C_{2}$ ), then again $\operatorname{tor}_{P} \oplus$ tor $_{\phi P}$ contributes at least $1 / 2+2 / n_{1}$ to $\sum a_{i} / n$. If $n_{1} \leqq 4$, we are done. If $n_{1} \geqq 6$, then one of the $C_{i}$, say $C_{1}$, is again elliptic with $j\left(C_{1}\right)=0$. If $n_{1}=6$, we conclude as before. And if $n_{1}>6$, the order of $\phi$ on $C_{2}$ must be 4 . But $P \in C_{2}$ satisfies $\phi P \neq P, \phi^{2} P=P$, so $C_{2}$ cannot be $\mathbf{P}^{1}$ (because the map $z \rightarrow \sqrt{-1} z$ on $\mathbf{P}^{1}$ has no points of order exactly 2). Thus $C_{2}$ must be elliptic with $j\left(C_{2}\right)=12^{3}$. Then $d z^{\otimes 2} \in H^{0}\left(o\left(2 K_{C_{2}}\right)\right)$ contributes $1 / 2$ to $\sum a_{i} / n$ and we are done. This completes the proof that $\phi$ fixes all double points of $C$.

We now go back to the list a)-e) and enumerate the possible components that $C$ can have.

Case a). Any $C_{\alpha}$ with $\left.\phi\right|_{C_{\alpha}}=$ identity.

Case b). $C_{\alpha}=\mathbf{P}^{1}$. In suitable coordinates on $C_{\alpha},\left.\phi\right|_{C_{\alpha}}$ is the map $z \mapsto \zeta z$. But all $P_{\beta} \in C_{\alpha}$ are either fixed by $\phi$ or of order 2. $\phi$ has only 2 fixed points and there are at least $3 P_{\beta}$ 's on $C_{\alpha}$. Thus at least one of the $P_{\beta}$, call it $P_{1}$, has order 2 . But $\phi$ has a point of order exactly 2 only if $\phi$ itself has order $2: \phi(z)=-z$. Now $P_{1}$ and $\phi P_{1}$ are mapped to the same double point $P$ of $C$ and because $\phi^{2}$ is the identity on $C_{\alpha}$, we see that $(C, \phi)$ near $P$ is given by $x y=0, \phi^{*} x=y, \phi^{*} y=x$. As above, this means that $(C, \phi)$ can be deformed to eliminate $P$, so this case is taken care of.

Case c). $C_{\alpha}$ is elliptic.

Case c1). $\left.\phi\right|_{C_{\alpha}}$ is a translation. Since $\phi^{2} P_{\beta}=P_{\beta}$ for all $\beta,\left.\phi\right|_{C_{\alpha}}$ has order 2 and $\phi^{2}=$ identity. Then as in case (b), the double points of $C$ which are images of these $P_{\beta}$ can be deformed away and this case is taken care of.

Case $c 2$ ). $\left.\phi\right|_{C_{\alpha}}$ fixes $0 \in C_{\alpha}$ and has order 2, i.e., it is the inverse in the group structure on $C_{\alpha}$. As $\left.\phi^{2}\right|_{C_{\alpha}}=$ identity, we are through as above if $\phi P_{\beta} \neq P_{\beta}$ for some $\beta$. Therefore we may assume all $P_{\beta}$ are points of order 2 . Now for any 2 points $P_{1}, P_{2} \in C_{\alpha}$ of order 2 ,

$$
H^{0}\left({ }^{C_{\alpha}}\left(P_{1}+P_{2}\right)\right)
$$

contains one even function, namely 1 , and one odd function, in fact one with a simple pole at each $P_{i}$. Thus

contributes eigenvalues

$$
H^{0}\left({ }^{\circ} C_{\alpha}\left(2 K_{C_{\alpha}}+\sum P_{\beta}\right)\right)
$$

$$
(0,1 / 2, \ldots, 1 / 2)
$$


to $\sum a_{i} / n$. Thus if there are 3 or $4 P_{\beta}$ 's, we are through, and we are left with 2 cases for $C_{\alpha}^{\prime}$ :

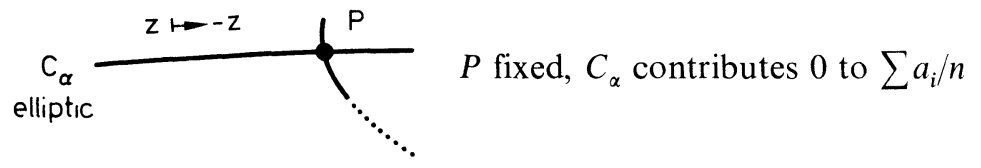

or

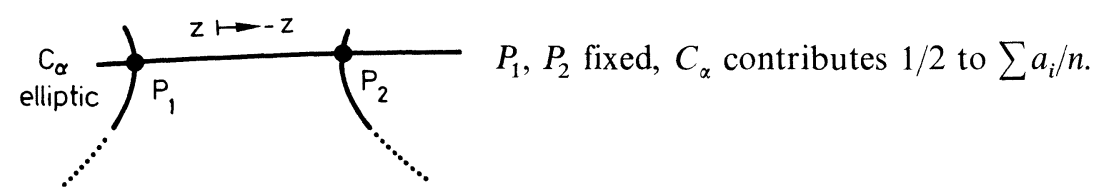

Case c3). $\left.\phi\right|_{C_{\alpha}}$ fixes $0 \in C_{\alpha}$ and has order 3 . Then $j(\alpha)=0$ and each $P_{\beta}$ is a fixed point. There are 3 of these. If 2 or 3 of these occur as $P_{\beta}$, we are done by considering the eigenvalues of $\phi$ on $H^{0}\left({ }^{\circ} C_{\alpha}\left(2 K_{\alpha}+P_{1}+P_{2}\right)\right)$. In fact, if $d z$ is the translation-invariant differential and $P_{1}=0$, then locally the 2 sections here look like

$$
d z^{\otimes 2}, z d z^{\otimes 2} \text {. }
$$

Under $z \mapsto \zeta_{3} z$, these give a contribution $\frac{1}{3}+\frac{2}{3}$ to $\sum a_{i} / n$. This leaves the case of one $P_{\beta}$, or $C_{\alpha}^{\prime}$ looking like:

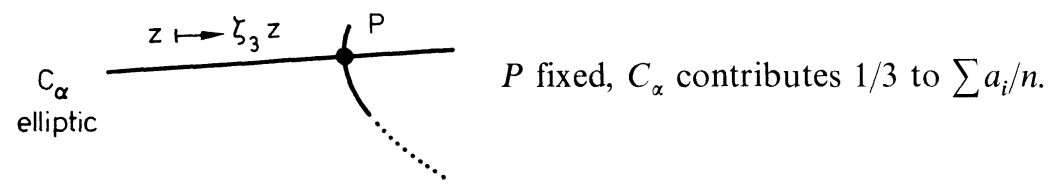

Case c 4$).\left.\phi\right|_{C_{\alpha}}$ fixes $0 \in C_{\alpha}$ and has order 4 . Then $j\left(C_{\alpha}\right)=12^{3}, \phi$ has 2 fixed points $0=P_{0}$ and $P_{1}$ and $\phi^{2}=$ inverse has 2 more fixed points $P_{2}$ and $\phi P_{2}$. Thus $\left\{P_{\beta}\right\}$ could consist in $\left\{P_{0}\right\},\left\{P_{0}, P_{1}\right\},\left\{P_{0}, P_{2}, \phi P_{2}\right\},\left\{P_{0}, P_{1}, P_{2}, \phi P_{2}\right\} .\left(\left\{P_{2}, \phi P_{2}\right\}\right.$ is impossible because $C_{\alpha}^{\prime}$ must meet the rest of $C$ somewhere). The eigenvalues on $H^{0}\left({ }^{\circ} C_{\alpha}\left(2 K_{C_{\alpha}}+\sum P_{\beta}\right)\right)$ are respectively $(1 / 2),(1 / 2,1 / 4),(1 / 2,1 / 4,3 / 4)$ in the first 3 cases, so the only cases where $\sum a_{i} / n<1$ could occur are the first 2 cases:

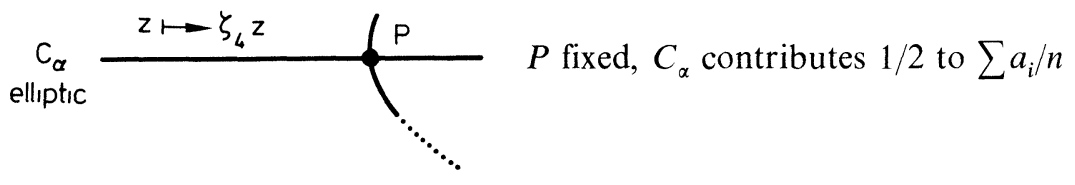

or

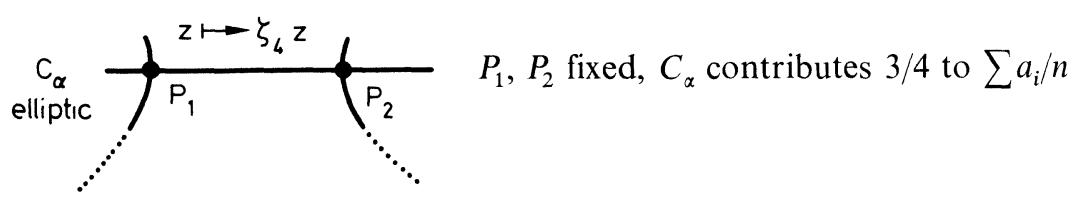


Case c 5). $\left.\phi\right|_{C_{\alpha}}$ fixes $0 \in C_{\alpha}$ and has order 6 . Then $j\left(C_{\alpha}\right)=0, \phi$ has only $0=P_{0}$ as fixed point, but $\phi^{2}$ has 2 further fixed points $P_{1}, \phi P_{1}$. The set $\left\{P_{\beta}\right\}$ is either $\left\{P_{0}\right\}$ or $\left\{P_{0}, P_{1}, \phi P_{1}\right\}$ and the eigenvalues for these are $\left(\frac{1}{3}\right),\left(\frac{1}{3}, \frac{2}{3}, \frac{1}{6}\right)$. (In fact, $d z^{\otimes 2} \in H^{0}\left({ }^{\circ} C_{\alpha}\left(2 K_{C_{\alpha}}\right)\right)$ is transformed under $z \mapsto \zeta_{6} z$ by $\zeta_{3}$; and a basis of $H^{0}\left({ }^{o} C_{\alpha}\left(2 K_{C_{\alpha}}+P_{0}+P_{1}+\phi P_{1}\right)\right)$ looks locally like $d z^{\otimes 2}, \frac{d z^{\otimes 2}}{z}$ and $z^{2} d z^{\otimes 2}$.) The only case to consider is therefore

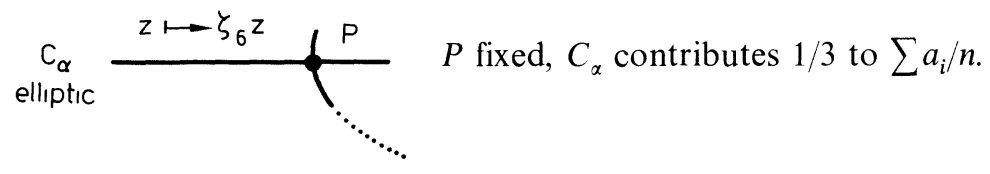

Case d). $C_{\alpha}$ is hyperelliptic of genus 2 or $3,\left.\phi\right|_{C_{\alpha}}=$ hyperelliptic involution. The set $\left\{P_{\beta}\right\}$ must consist in at least one fixed point of $\phi$, i.e., a Weierstrass point of $C_{\alpha}$ and possibly further Weierstrass points or pairs $P, \phi P$. But the dimension of the $(-1)$-eigenspace of $\phi$ on $H^{0}\left(o\left(2 K_{C}+\sum P_{\beta}\right)\right)$ is always $\geqq 2$ if $g=3$ and $P_{1}$ is Weierstrass point. And it is 1 if $g=2$ and $\left\{P_{\beta}\right\}$ consists in a single Weierstrass point, $\geqq 2$ if $g=2$ and there are further $P$. So the only case not eliminated is

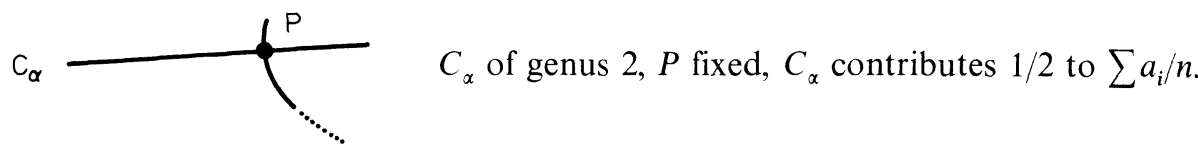

Case $e$ ). $C$ of genus 2, double cover of elliptic curve, $\phi=$ sheet interchange. If $P$ is a fixed point of $\phi$, then the dimension of the $(-1)$-eigenspace of $\phi$ on $H^{0}\left(a\left(2 K_{C}+P\right)\right)$ is 2 , giving $\left(\frac{1}{2}, \frac{1}{2}\right)$ to $\left\{a_{i} / n\right\}$. So this case is eliminated.

We are left with a problem of patching together the few curves of genus 1 and 2 above with a lot of $C_{\alpha}$ 's where $\phi$ acts identically. In each curve so obtained, we must add up all the contributions to $\sum a_{i} / n$. One notes that the following lead to $\sum a_{i} / n \geqq 1$ :

i)

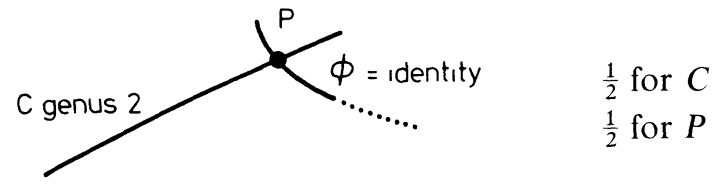

ii)

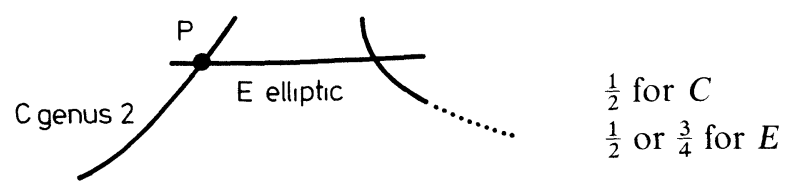

iii)

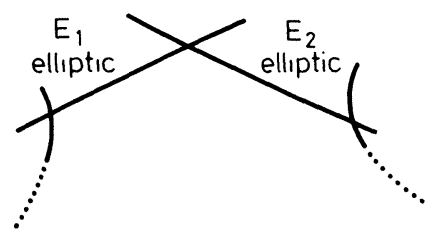

$\frac{1}{2}$ or $\frac{3}{4}$ for $E_{1}, E_{2}$ 
iv)

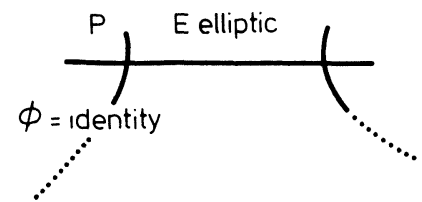

$\frac{1}{2}$ for $E \quad \frac{3}{4}$ for $E$

$\frac{1}{2}$ for $P$ or $\geqq \frac{1}{4}$ for $P$

Excluding cases where $g \leqq 3$, it's easy to see that we are left with "the elliptic tails":

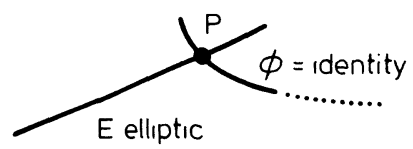

Finally, if $\left.\phi\right|_{E}$ has order 3 , one finds that $E$ contributes $1 / 3, P 2 / 3$ so this gives $\sum a_{i} / n \geqq 1$ too. Q.E.D.

By Reid-Tai's criterion, it now follows that an $n$-canonical form $\omega$ on $\overline{\mathscr{M}}_{g}^{0}$ is holomorphic on $\tilde{\tilde{M}_{g}}$ provided it is holomorphic on the resolution of a neighborhood of the following 3 points in $\overline{\mathscr{M}}_{\mathrm{g}}$ :

a) the generic curve $C=C_{1} \cup C_{2}, C_{1} \cap C_{2}=\{P\}, C_{i}$ of genus $g-1, C_{2}$ elliptic,

b) the generic curve $C=C_{1} \cup C_{2}, C_{1} \cap C_{2}=\{P\}, C_{1}$ of genus $g-1, C_{2}$ elliptic with $j\left(C_{2}\right)=0$,

c) the generic curve $C=C_{1} \cup C_{2}, C_{1} \cap C_{2}=\{P\}, C_{1}$ of genus $g-1, C_{2}$ elliptic with $j\left(C_{2}\right)=12^{3}$.

In fact, we now take any smooth curve $C_{1}$ without automorphisms and a point $P \in C_{1}$ and consider the curve in $\overline{\mathscr{M}}_{\mathrm{g}}$ parametrizing the stable curves $C_{1} \cup C_{2}$, where $C_{1} \cap C_{2}=\{P\}$ and $C_{2}$ is any elliptic curve or is a rational curve with node. We shall show that any $\omega$ holomorphic on $\overline{\mathscr{M}}_{g}^{0}$ is also holomorphic on the resolution of a neighborhood of this curve.

To do this, let $C_{0}$ be the curve obtained from $C_{1}$ by making $P$ into an ordinary cusp $P_{0} \in C_{0}$, i.e.,

$$
{ }^{0} C_{0}=k+m_{P, C}^{2} .
$$

Consider the universal deformation space:

$$
\mathscr{C} \rightarrow \Delta^{3 g-3}
$$

of $C_{0}$. Since any deformation of $C_{0}$ induces a deformation of a neighborhood of $P_{0}, \Delta^{3 g-3}$ fibres over $\Delta^{2}$, the base space of the universal deformation of a cusp. More precisely, there are coordinates $t_{1}, t_{2}, t_{3}, \ldots, t_{3 g-3}$ on $\Delta^{3 g-3}$ and coordinates $x, y, t_{1}, \ldots, t_{3 g-3}$ on $\mathscr{C}$ near $P_{0}$ such that

i) $C_{0}$ is given by $y^{2}=x^{3}, t_{i}=0,|x|,|y|<\varepsilon$, near $P_{0}$,

ii) more generally, $\mathscr{C}$ is given by

$$
y^{2}=x^{3}+t_{1} x+t_{2}, \quad|x|,|y|<\varepsilon .
$$


Outside of the codimension 2 set $V\left(t_{1}, t_{2}\right)$ (i.e., $\left.t_{1}=t_{2}=0\right) \mathscr{C}$ is a family of stable curves of genus $g$. Moreover, the fibres of $\mathscr{C}$ are all irreducible curves which either have one cusp, one node, or are smooth. Considering

$$
\operatorname{Isom}_{\Delta^{3 g-3}}(\mathscr{C}, \mathscr{C})=\left\{\begin{array}{c}
\text { scheme of triples } s_{1}, s_{2}, \phi, \\
s_{1}, s_{2} \in \Delta^{3 g-3}, \\
\phi: C_{s_{1}} \stackrel{\simeq}{\longrightarrow} C_{s_{2}} \text { an isomorphism }
\end{array}\right\}
$$

we see that because $C_{0}$ has no automorphisms, all nearby curves in the family $\mathscr{C}$ have no automorphisms and occur only once in the family. Therefore we have a holomorphic map

$$
f: \Delta^{3 g-3}-V\left(t_{1}, t_{2}\right) \rightarrow \overline{\mathscr{M}}_{\mathrm{g}}^{0}
$$

which is injective, and therefore an isomorphism of $\Delta^{3 g-3}-V\left(t_{1}, t_{2}\right)$ with an open subset of $\overline{\mathscr{M}}_{\mathrm{g}}^{0}$. We want to study the singularity of $f$ at $V\left(t_{1}, t_{2}\right)$. To do this, we want to convert $\mathscr{C} / \Delta^{3 g-3}$ into a family of stable curves.

Let

$$
S \rightarrow \Delta^{3 g-3}
$$

be the normalization of the blow up of the ideal $\left(t_{1}^{3}, t_{2}^{2}\right)$. We shall show that $f$ extends to an isomorphism $\tilde{f}$ of $S$ with a suitable open set in $\mathscr{M}_{g}$. $S$ is covered by 2 charts:

$$
\begin{aligned}
& S_{1} \text { with coordinates } t_{1}, t_{2}, \frac{t_{1}^{2}}{t_{2}}, \frac{t_{1}^{3}}{t_{2}^{2}}, \\
& S_{2} \text { with coordinates } t_{1}, t_{2}, \frac{t_{2}}{t_{1}}, \frac{t_{2}^{2}}{t_{1}^{3}} .
\end{aligned}
$$

Let $p_{1}: \tilde{S}_{1} \rightarrow S_{1}$ be the normalization of $S_{1}$ in the 6-cyclic covering $u_{2}=t_{2}^{1 / 6}$. Then $\tilde{S}_{1}$ is smooth with coordinates $u_{1}, u_{2}, t_{3}, \ldots, t_{3 g-3}, p_{1}$ being given by

$$
\begin{aligned}
t_{1} & =u_{1} u_{2}^{4}, \\
t_{2} & =u_{2}^{6}, \\
\left(t_{1}^{2} / t_{2}\right) & =u_{1}^{2} u_{2}^{2}, \\
\left(t_{1}^{3} / t_{2}^{2}\right) & =u_{1}^{3} .
\end{aligned}
$$

Moreover, the group $\mu_{6}$ of $6^{\text {th }}$ roots of 1 acts on $\tilde{S}_{1}$ by

$$
\left(u_{1}, u_{2}\right) \mapsto\left(\zeta^{2} u_{1}, \zeta u_{2}\right)
$$

so that $S_{1} \cong \tilde{S}_{1} / \mu_{6}$. Let $p_{2}: \tilde{S}_{2} \rightarrow S_{2}$ be the normalization of $S_{2}$ in the 4-cyclic covering $u_{1}=t_{1}^{1 / 4}$. Then $\tilde{S}_{2}$ is smooth with coordinates $u_{1}, u_{2}, t_{3}, \ldots, t_{3 g-3}, p_{2}$ being given by 


$$
\begin{aligned}
t_{1} & =u_{1}^{4}, \\
t_{2} & =u_{2} u_{1}^{6}, \\
t_{2} / t_{1} & =u_{2} u_{1}^{2}, \\
t_{2}^{2} / t_{1}^{3} & =u_{2}^{2} .
\end{aligned}
$$

Moreover, the group $\boldsymbol{\mu}_{4}$ of $4^{\text {th }}$ roots of 1 acts on $\tilde{S}_{2}$ by

so that $S_{2}=\tilde{S}_{2} / \mu_{4}$.

$$
\left(u_{1}, u_{2}\right) \mapsto\left(\zeta u_{1}, \zeta^{2} u_{2}\right)
$$

Now pull the family $\mathscr{C} \rightarrow \Delta^{3 g-3}$ back to $\tilde{S}_{1}$ and $\tilde{S}_{2}$. Over $\tilde{S}_{1}$, the family is given near $P_{0}$ by

$$
y^{2}=x^{3}+u_{1} u_{2}^{4} x+u_{2}^{6}
$$

Let $\mathscr{C}_{1}$ be the normalization of the blow-up of $\mathscr{C} \times \times_{\left(A^{38}-3\right)} \tilde{S}_{1}$ in the ideal $\left(x, u_{2}^{2}\right)$. It is covered by the 2 charts

$$
\begin{gathered}
\mathscr{C}_{1, a}: y^{\prime 2}=x^{\prime 3}+u_{1} x^{\prime}+1, \text { where } x^{\prime}=x / u_{2}^{2}, y^{\prime}=y / u_{2}^{3} \\
\mathscr{C}_{1, b}: x^{\prime \prime} \cdot y^{\prime \prime}=u_{2}, \text { where } x^{\prime \prime}=u_{2} x / y,\left|x^{\prime \prime}\right|,\left|y^{\prime \prime}\right|<\varepsilon, y^{\prime \prime}=y / x \\
\left(\text { note that: } y^{\prime \prime 2}=x\left(1+u_{1}\left(\frac{u_{2}^{2}}{x}\right)^{2}+\left(\frac{u_{2}^{2}}{x}\right)^{3}\right)\right. \\
x^{\prime \prime 2}=\frac{\left(u_{2}^{2} / x\right)}{\left(1+u_{1}\left(u_{2}^{2} / x\right)^{2}+\left(u_{2}^{2} / x\right)^{3}\right)}
\end{gathered}
$$

so that $x^{\prime \prime}, y^{\prime \prime}$ are integrally dependent on $x, u_{1}, \frac{u_{2}^{2}}{x}$ when $\left|\frac{u_{2}^{2}}{x}\right|<\varepsilon$.)

In particular, the fibre of $\mathscr{C}_{1}$ over points where $u_{2}=0$ looks like:

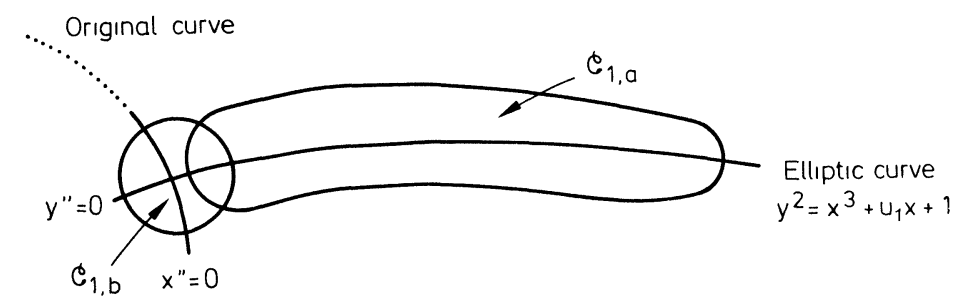

Moreover, $\mathscr{C}_{1}$ is a smooth variety, fibred in stable curves over $\tilde{S}_{1}$. The same works for the pull-back of $\mathscr{C}$ to $\tilde{S}_{2}$, which is given near $P_{0}$ by

$$
y^{2}=x^{3}+u_{1}^{4} x+u_{2} u_{1}^{6}
$$

Let $\mathscr{C}_{2}$ be the normalization of the blow-up of $\mathscr{C} \times \times_{\left(4^{3} g-3\right)} \tilde{S}_{2}$ in the ideal $\left(x, u_{1}^{2}\right)$. It is covered by the 2 charts: 


$$
\begin{gathered}
\mathscr{C}_{2, a}: y^{\prime 2}=x_{1}^{\prime 3}+x^{\prime}+u_{2} \quad \text { where } y^{\prime}=y / u_{1}^{3}, x^{\prime}=x / u_{1}^{2}, \\
\mathscr{C}_{2, b}: x^{\prime \prime} y^{\prime \prime}=u_{1}, \quad \text { where } x^{\prime \prime}=\frac{x u_{1}}{y}, y^{\prime \prime}=\frac{y}{x},\left|x^{\prime \prime}\right|,\left|y^{\prime \prime}\right|<\varepsilon \\
\left(\text { note that } y^{\prime \prime 2}=x\left(1+\left(\frac{u_{1}^{2}}{x}\right)^{2}+u_{2}\left(\frac{u_{1}^{2}}{x}\right)^{3}\right)\right. \\
x^{\prime \prime 2}=\frac{u_{1}^{2} / x}{1+\left(u_{1}^{2} / x\right)^{2}+u_{2}\left(u^{2} / x\right)^{3}}
\end{gathered}
$$

so that $x^{\prime \prime}, y^{\prime \prime}$ are integrally dependent on $x, u_{2}, u_{1}^{2} / x$ when $\left|u_{1}^{2} / x\right|<\varepsilon$.)

Thus $\mathscr{C}_{2}$ has the same form as $\mathscr{C}_{1}$, except that the elliptic "tail" is now:

$$
y^{2}=x^{3}+x+u_{2} .
$$

It follows that the map $f$ extends to holomorphic maps $\tilde{f}_{1}: \tilde{S}_{1} \rightarrow \overline{\mathscr{M}}_{\mathrm{g}}, \tilde{f}_{2}$ : $\tilde{S}_{2} \rightarrow \overline{\mathscr{M}}_{\mathrm{g}}$ in the diagram:

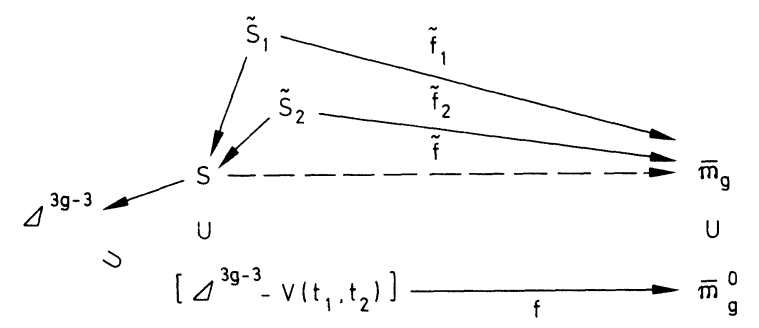

But the action of $\boldsymbol{\mu}_{6}$ on $\tilde{S_{1}}$ lifts to an action on $\mathscr{C}_{1}$ :

$$
\begin{aligned}
\left(x^{\prime}, y^{\prime}\right) & \mapsto\left(\zeta^{4} x^{\prime}, \zeta^{3} y^{\prime}\right), \\
\left(x^{\prime \prime}, y^{\prime \prime}\right) & \mapsto\left(\zeta x^{\prime \prime}, y^{\prime \prime}\right)
\end{aligned}
$$

and the action of $\mu_{4}$ on $\tilde{S}_{2}$ lifts to an action on $\mathscr{C}_{2}$ :

$$
\left(x^{\prime}, y^{\prime}\right) \mapsto\left(\zeta^{2} x^{\prime}, \zeta y^{\prime}\right)
$$

Thus $\tilde{f}_{1}, \tilde{f}_{2}$ factor through

$$
\left(x^{\prime \prime}, y^{\prime \prime}\right) \mapsto\left(\zeta x^{\prime \prime}, y^{\prime \prime}\right) \text {. }
$$

$$
\tilde{f}: S \rightarrow \bar{M}_{g}
$$

Examining the fibres, we see that $\tilde{f}$ is injective, which proves:

Lemma. $S$ is isomorphic to a neighborhood in $\overline{\mathscr{M}}_{\mathrm{g}}$ of the curve parametrizing the set of stable curves:

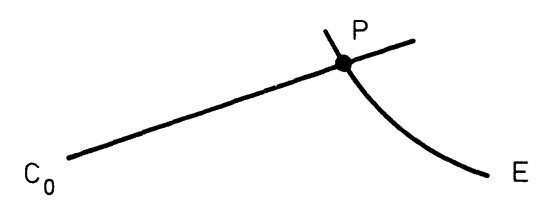

where $E$ is an arbitrary elliptic curve or rational curve. 
It now follows from this lemma that any holomorphic tensor on $\overline{\mathscr{M}}_{\mathrm{g}}^{0}$

a) restricts to $\Delta^{3 g-3}-V\left(t_{1}, t_{2}\right)$

b) extends holomorphically to $\Delta^{3 \mathrm{~g}-3}$, since $V\left(t_{1}, t_{2}\right)$ has codimension 2

c) pulls back holomorphically to a resolution of $S$, hence

d) extends holomorphically to a resolution of $\overline{\mathscr{M}}_{\mathrm{g}}$ over all points made up of a smooth curve $C_{0}$ without automorphisms joined at one point to an elliptic curve or nodal rational curve.

Together with the Reid-Tai criterion, this completes the proof of Theorem 1.

\section{Appendix 1 to $§ 1$}

Reid-Tai's Criterion. Let $V$ be a vector space of dimension $v, G \subset G L(V)$ a finite group. For all $g \in G$ of order $n$, let the eigenvalues of $g$ be $\zeta^{a_{1}}, \ldots, \zeta^{a_{v}}$, where $\zeta$ is a primitive $n^{\text {th }}$ root of 1 and $0 \leqq a_{i}<n$. Moreover, let $V^{0} \subset V$ be the open set where $G$ acts freely, and let $\omega$ be an $m$-canonical differential on $V^{0} / G$. Then $\omega$ extends holomorphically to a resolution $V \widetilde{T G}$ of $V / G$ if :

for all $g \in G$ such that $\sum a_{i} / n<1$ for some choice of $\zeta, \omega$ is holomorphic along all divisors $E \subset V / G$ mapping onto the image in $V / G$ of the fixed point set $V^{g}$.

Proof. We first reduce the result to the special case $G$ cyclic. In fact, let $p$ : $\tilde{V} \rightarrow V / G$ be the normalization of $V \widetilde{T}_{G}$ in the function field $\mathbf{C}(V)$. Then $\omega$ is holomorphic on $V \widetilde{T}$ if $\omega$ has no poles on any divisor $E \subset V / G$. But over each divisor $E$, the covering $\tilde{V} \rightarrow V \widetilde{T}$ has a cyclic ramification subgroup, i.e., for all components $E_{1}$ of $p^{-1} E$, there is a cyclic subgroup $H \subset G$ fixing $E_{1}$ identically such that in an $H$-stable Zariski-open subset $U_{E} \subset \tilde{V}$ meeting $E_{1}, p$ factors:

$$
U_{E} \longrightarrow U_{E} / H \stackrel{\text { étale }}{p_{E}} V / \widetilde{T} \text {. }
$$

In particular, $\omega$ is regular on $E$ if $p_{E}^{*} \omega$ is regular on $E_{1}$. But if the criterion holds for $G$, it also holds for $H$, hence $\omega$ is regular on $U_{E} / H$, hence $\omega$ is regular on $E$.

Now assume $G$ is cyclic, $g \in G$ is a generator and that $X_{1}, \ldots, X_{v}$ are coordinates such that $g^{*} X_{i}=\zeta^{a_{i}} X_{i}$. Then $G$ is contained in the torus $\mathbf{G}_{m}^{v}$ of all diagonal automorphisms of $V$, and we may assume that the resolution $V / G$ is $\mathbf{G}_{m}^{v}$-equivariant. Now $\widetilde{V / G}$ is a smooth equivariant partial compactification of the torus $\mathbf{G}_{m}^{v} / G$. By the theory of torus embeddings, each divisor $E$ of $V / G$ $-\left(\mathbf{G}_{m}^{v} / G\right)$, hence each component $E$ of $V \widetilde{G}-V^{0} / G$, determines a monomorphism:

such that

$$
\lambda: \mathbf{G}_{m} \rightarrow \mathbf{G}_{m}^{v} / G
$$

a) if $X^{\alpha}$ is a character of $\mathbf{G}_{m}^{v} / G$ such that $X^{\alpha} \circ \lambda$ vanishes to order 1 at $0 \in \overline{\mathbf{G}}_{m}$, then $X^{\alpha}$ is a local equation almost everywhere for $E$,

b) the function field $\mathbf{C}(E)$ of $E$ is generated by the restrictions of the characters $X^{\alpha}$ such that $X^{\alpha} \circ \lambda \equiv 1$. 
c) $\lim _{t \rightarrow 0} \lambda(t)(a) \in E$ for all $a \in \mathbf{G}_{m}^{v} / G$.

The homomorphisms $\lambda$ may be described by

where

$$
\lambda^{*}\left(X_{i}\right)=t^{\ell_{i}}, \quad 1 \leqq i \leqq v
$$

$$
\left(\ell_{1}, \ldots, \ell_{v}\right) \in \mathbf{Z}^{v}+\mathbf{Z}\left(\frac{a_{1}}{n}, \ldots, \frac{a_{v}}{n}\right),
$$

and if $\lambda$ is associated to $E \subset \widetilde{V / G}$, then $\ell_{i} \geqq 0,1 \leqq i \leqq v$.

Now let

$$
\omega=\left(\sum_{\alpha \geqq 0} c_{\alpha} X^{\alpha}\right)\left(d X_{1} \wedge \ldots \wedge d X_{v}\right)^{\otimes m}
$$

be the given $m$-canonical differential. Write

$$
\begin{aligned}
\omega & =\left(\sum_{\alpha \geqq 0} c_{\alpha} X^{\alpha+m \varepsilon}\right)\left(\frac{d X_{1}}{X_{1}} \wedge \ldots \wedge \frac{d X_{v}}{X_{v}}\right)^{\otimes m}, \\
\varepsilon & =(1, \ldots, 1) .
\end{aligned}
$$

Let $Y_{i}=X^{\beta_{i}}, 1 \leqq i \leqq v$, be a basis of the character group of $\mathbf{G}_{m}^{v} / G$ such that $Y_{1} \circ \lambda$ vanishes to order 1 at $t=0, Y_{i} \circ \lambda \equiv 1,2 \leqq i \leqq v$. Then

$$
\frac{d Y_{1}}{Y_{1}} \wedge \ldots \wedge \frac{d Y_{v}}{Y_{v}}=\text { (const.) } \frac{d X_{1}}{X_{1}} \wedge \ldots \wedge \frac{d X_{v}}{X_{v}},
$$

hence $\omega$ is holomorphic along $E$ if and only if

$$
c_{\alpha} \neq 0 \Rightarrow X^{\alpha+m \varepsilon}=Y_{1}^{r_{1}} \ldots Y_{v}^{r_{v}} \quad \text { where } r_{1} \geqq m .
$$

But $r_{1}$ is just the order of vanishing of $X^{\alpha+m \varepsilon} \circ \lambda$ at $t=0$, i.e.,

$$
r_{1}=\left\langle\alpha+m \varepsilon,\left(\ell_{1}, \ldots, \ell_{v}\right)\right\rangle
$$

But then as $\alpha_{i} \geqq 0, \ell_{i} \geqq 0$, we get

Now

$$
r_{1} \geqq m\left(\sum \ell_{i}\right) .
$$

Note that

$$
\ell_{i}=k_{i}+k a_{i} / n, \quad \text { some } k, k_{i} \in \mathbf{Z} \text {. }
$$

Let

$$
\left(g^{k}\right)^{*} X_{i}=\zeta^{k a_{2}} X_{i}=\zeta^{n k_{i}+k a_{i}} X_{i}=\zeta^{n \ell_{i}} X_{i}
$$

so that

$$
n \ell_{i} \equiv a_{i}^{\prime}(\bmod n), \quad 0 \leqq a_{i}^{\prime}<n
$$

If

$$
\left(g^{k}\right)^{*} X_{i}=\zeta^{a_{i}^{\prime}} X_{i}
$$

it follows that

$$
\sum a_{i}^{\prime} / n \geqq 1,
$$

$$
\sum \ell_{i} \geqq \sum \frac{a_{i}^{\prime}}{n} \geqq 1,
$$


hence $r_{1} \geqq m$ as required. We are also done if $\ell_{i} \geqq 1$ for any $i$. Now assume $\sum \frac{a_{i}^{\prime}}{n}<1$ and $\ell_{i}<1$, all $i$. Then $g^{k}$ is one of the "bad" elements of $G$, so that by the assumption, $\omega$ is holomorphic on those $E$ 's over the fixed point set of $V^{\left(\mathrm{g}^{k}\right)}$. But

$$
\begin{aligned}
V^{\left(\mathrm{g}^{k}\right)} & =\left\{\left(X_{1}, \ldots, X_{v}\right) \mid X_{i} \neq 0 \Rightarrow a_{i}^{\prime}=0\right\} \\
& =\left\{\left(X_{1}, \ldots, X_{v}\right) \mid X_{i} \neq 0 \Rightarrow \ell_{i} \in \mathbf{Z}\right\} \\
& =\left\{\left(X_{1}, \ldots, X_{v}\right) \mid X_{i} \neq 0 \Rightarrow \ell_{i}=0\right\} .
\end{aligned}
$$

By property $(c)$ above, $E$ maps onto $V^{\left(g^{k}\right)}$, so we are done for this $E$ too. Q.E.D.

\section{Appendix 2 to $§ 1$}

The following BASIC program which was run on the second author's Apple II looks one at a time at all cyclic covers of $\mathbf{P}^{1}$ branched in $0,1, \infty$. These are all given by

$$
y^{n}=x^{a}(x-1)^{b} .
$$

We may assume, permuting the branch points if necessary, that

$$
\begin{gathered}
1 \leqq a \leqq \frac{n}{3}, \quad a \mid n, \\
a \leqq b \leqq \frac{n-a}{2}, \quad(a, b)=1 .
\end{gathered}
$$

The program calculates the genus $g$ which by Hurwitz' formula is:

$$
g=\frac{n-(n, a)-(n, b)-(n, a+b)}{2}+1 .
$$

It can be checked that $n \leqq 4 g+2$. If $H^{0}\left({ }_{o_{c}}(2 K)\right)$ is decomposed into eigenspaces $V_{i}, 0 \leqq i<n$, under $\phi:(x, y) \rightarrow\left(x, e^{2 \pi i / n} y\right)$, then the program calculates $e(i)=\operatorname{dim} V_{i}$ and for all $j, 1 \leqq j \leqq n-1,(j, n)=1$, it calculates $\sum_{1}^{3 g-3} a_{i}$ for the automorphism $\phi^{j}$ : this is the variable $z$. The max and min over all $j$ are called $z a, z b$, and the max and $\min$ of $\sum a_{i} / n$ for each $g$ for curves studied so far are kept in the arrays $\min (g), \max (g)$.

\section{]LIST}

1 G0=18: REM G0 IS THE MAXIMUM GENUS WHICH WILL BE CONSIDERED

10 DIM E(500): DIM MIN(100): DIM MAX(100)

20 FOR $\mathrm{G}=2$ TO G0

$30 \mathrm{MIN}(\mathrm{G})=1000000$ 
$35 \operatorname{MAX}(\mathrm{G})=0$

40 NEXT G

100 FOR $\mathrm{N}=3$ TO $(4 * \mathrm{G} 0)+2$ : REM N IS THE ORDER OF THE CYCLIC COVER

105 REM WE LOOK AT THE N-TH ROOT OF $(\mathrm{X} * * \mathrm{~A}) *((\mathrm{X}-1) * * \mathrm{~B})$

110 FOR $\mathrm{A}=1$ TO INT(N/3)

$115 \mathrm{X}=\mathrm{N}: \mathrm{Y}=\mathrm{A}:$ GOSUB $1000: \mathrm{D} 1=\mathrm{X}$

120 IF (D1 < A) THEN GOTO 510: REM WE WANT A TO DIVIDE N

$130 \quad \mathrm{FOR} B=\mathrm{A}$ TO INT $((\mathrm{N}-\mathrm{A}) / 2)$

$150 \mathrm{X}=\mathrm{D} 1: \mathrm{Y}=\mathrm{B}$ : GOSUB 1000

160 IF $X>1$ THEN GOTO 500: REM WE WANT G.C.D. (A, B) $=1$

$170 \quad \mathrm{X}=\mathrm{N}: \mathrm{Y}=\mathrm{B}: \mathrm{GOSUB}$ 1000: $\mathrm{D} 2=\mathrm{X}$

$180 \quad \mathrm{X}=\mathrm{N}: \mathrm{Y}=\mathrm{A}+\mathrm{B}:$ GOSUB 1000: $\mathrm{D} 3=\mathrm{X}$

$190 \mathrm{G}=(\mathrm{N}-\mathrm{D} 1-\mathrm{D} 2-\mathrm{D} 3) / 2+1$ : REM G IS THE GENUS OF THE COVER

192 IF $\mathrm{G}<2$ THEN GOTO 500

193 IF G $>$ G0 THEN GOTO 500

$200 \quad$ FOR $\mathrm{I}=1$ TO N -1

$210 \mathrm{E}(\mathrm{I})=\mathrm{INT}((\mathrm{I} * \mathrm{~A}-2 * \mathrm{D} 1) / \mathrm{N})+\mathrm{INT}((\mathrm{I} * \mathrm{~B}-2 * \mathrm{D} 2) / \mathrm{N})$ $+\operatorname{INT}((-\mathrm{I} *(\mathrm{~A}+\mathrm{B})-2 * \mathrm{D} 3) / \mathrm{N})+3$

215 IF E(I) $<0$ THEN E(I) $=0$

230 NEXT I

245 REM E(I) IS THE DIMENSION OF THE I-TH EIGENSPACE IN THE QUADRATIC DIFFERENTIALS

250 FOR $\mathrm{J}=1$ TO $\mathrm{N}-1$ : REM WE LOOK AT THE $\mathrm{J}$-TH POWER OF THE AUTOMORPHISM

$255 \mathrm{X}=\mathrm{N}: \mathrm{Y}=\mathrm{J}$ : GOSUB 1000

256 IF $X>1$ GOTO 320: REM WE WANT G.C.D. $(\mathrm{N}, \mathrm{J})=1$

$260 \quad \mathrm{Z}=0$

$270 \quad$ FOR I $=1$ TO N -1

$280 \mathrm{~K}=\mathrm{J} * \mathrm{I}-\mathrm{N} * \mathrm{INT}(\mathrm{J} * \mathrm{I} / \mathrm{N})$

$290 \mathrm{Z}=\mathrm{Z}+\mathrm{K} * \mathrm{E}(\mathrm{I}): \mathrm{REM} \mathrm{Z}$ IS WHAT WE WROTE ABOVE AS THE SUM OF ASUBI

295 NEXT I

$298 \quad$ IF J $>1$ GOTO 300

$299 \mathrm{ZA}=\mathrm{Z}: \mathrm{ZB}=\mathrm{Z}:$ GOTO 320

300 IF $(Z A<Z)$ THEN $Z A=Z$ : IF $(Z B>Z)$ THEN $Z B=Z$

320 NEXT J

$330 \quad$ IF $(\mathrm{ZB} / \mathrm{N})<\mathrm{MIN}(\mathrm{G})$ THEN $\operatorname{MIN}(\mathrm{G})=\mathrm{ZB} / \mathrm{N}$

340 IF $(\mathrm{ZA} / \mathrm{N})>\operatorname{MAX}(\mathrm{G}) \operatorname{THEN} \operatorname{MAX}(\mathrm{G})=\mathrm{ZA} / \mathrm{N}$

500 NEXT B

510 NEXT A

520 NEXT N

600 FOR $\mathrm{G}=2$ TO G0

610 PRINT G; "MIN ="; $\operatorname{MIN}(\mathrm{G})$; "MAX ="; $\operatorname{MAX}(\mathrm{G})$

620 NEXT G

630 STOP 
1000 REM THIS SUBROUTINE STARTS WITH TWO INTEGERS X, Y AND CHANGES X TO THEIR G.C.D.

1005 IF $Y<=X$ GOTO 1020

$1010 Z=X: X=Y: Y=Z$

1020 IF $Y=0$ THEN RETURN

$1030 \mathrm{Z}=\mathrm{X}-\mathrm{Y} * \mathrm{INT}(\mathrm{X} / \mathrm{Y})$

$1040 \mathrm{X}=\mathrm{Y}: \mathrm{Y}=\mathrm{Z}$

1050 GOTO 1020

The program gave the following output; where the first number on each line is the genus, the second the $\min$ of $\sum a_{i} / n$, the third the max of $\sum a_{i} / n$ :

$\begin{array}{rl}2 & \mathrm{MIN}=1.2 \mathrm{MAX}=1.8 \\ 3 & \mathrm{MIN}=2.35714285 \mathrm{MAX}=3.64285715 \\ 4 & \mathrm{MIN}=3.55555556 \mathrm{MAX}=5.44444444 \\ 5 & \mathrm{MIN}=4.77272727 \mathrm{MAX}=7.22727273 \\ 6 & \mathrm{MIN}=6 \mathrm{MAX}=9 \\ 7 & \mathrm{MIN}=7.23333333 \mathrm{MAX}=10.7666667 \\ 8 & \mathrm{MIN}=8.47058824 \mathrm{MAX}=12.5294118 \\ 9 & \mathrm{MIN}=9.71052632 \mathrm{MAX}=14.2894737 \\ 10 & \mathrm{MIN}=10.952381 \mathrm{MAX}=16.047619 \\ 11 & \mathrm{MIN}=12.1956522 \mathrm{MAX}=17.8043478 \\ 12 & \mathrm{MIN}=13.44 \mathrm{MAX}=19.56 \\ 13 & \mathrm{MIN}=14.6851852 \mathrm{MAX}=21.3148148 \\ 14 & \mathrm{MIN}=15.9310345 \mathrm{MAX}=23.0689655 \\ 15 & \mathrm{MIN}=17.1774194 \mathrm{MAX}=24.8225806 \\ 16 & \mathrm{MIN}=18.4242424 \mathrm{MAX}=26.5757576 \\ 17 & \mathrm{MIN}=19.6714286 \mathrm{MAX}=28.3285714 \\ 18 & \mathrm{MIN}=20.9189189 \mathrm{MAX}=30.0810811\end{array}$

Break in 630

The regular growth of $\min \sum a_{i} / n$ and $\max \sum a_{i} / n$ indicates that there is some simple proposition at work, but we have not investigated this. All we care about here is that $\min \sum a_{i} / n \geqq 1$.

\section{§2. The Canonical Divisor Class on $\overline{\mathscr{M}}_{\mathrm{g}}$}

As in the previous section, $\overline{\mathscr{M}}_{\mathrm{g}}^{0}$ is the open set of $\overline{\mathscr{M}}_{\mathrm{g}}$ parametrizing curves without automorphisms. The components of $\overline{\mathscr{M}}_{\mathrm{g}}-\overline{\mathscr{M}}_{\mathrm{g}}^{\mathrm{o}}$ of codimension 2 or more are simply the components of the singular locus of $\overline{\mathscr{M}}_{g}$. But $\mathscr{M}_{g}-\overline{\mathscr{M}}_{g}^{0}$ has one component of codimension 1 : namely, the locus $\Delta_{1}$ of curves with "elliptic tails" encountered in the previous section. We shall first calculate the canonical divisor $K_{\mathscr{M}_{\mathrm{g}}}$ on the open set $\overline{\mathscr{M}}_{\mathrm{g}}^{0}$, and afterwards, indicate how to modify the calculation to give the canonical divisor $K_{\mathscr{M}_{g}}$ on the full open set of smooth points of $\overline{\mathscr{M}}_{g}$. 
As before, let

$$
\pi: \overline{\mathscr{C}}_{\mathrm{g}}^{0} \rightarrow \overline{\mathscr{M}}_{\mathrm{g}}^{0}
$$

be the universal family of stable, automorphism-free curves. We follow the technique in [13], pp.99-102 and apply Grothendieck's relative RiemannRoch theorem to the morphism $\pi$. For all coherent sheaves $\mathscr{F}$ on $\overline{\mathscr{C}}_{\mathrm{g}}^{0}$,

$$
\operatorname{ch}\left(\pi_{!} \mathscr{F}\right)=\pi_{*}\left(\operatorname{ch}(\mathscr{F}) \cdot T\left(\Omega_{\mathscr{C} / \mathcal{M}}^{1}\right)\right) \quad \text { in } A\left(\overline{\mathscr{M}}_{\mathrm{g}}^{0}\right) \otimes \mathbf{Q} .
$$

We wish to apply this for

In this case,

$$
\mathscr{F}=\Omega_{\mathscr{C} / \mathcal{M}}^{1} \otimes \omega_{\mathscr{C} / \mathscr{M}}
$$

$$
\pi_{*} \mathscr{F} \cong T_{\mathscr{M}_{\mathrm{g}}^{0}}^{*}, \quad R^{1} \pi_{*} \mathscr{F}=(0)
$$

because the cotangent space to $\overline{\mathscr{M}}_{\mathrm{g}}^{0}$ at every point $x$ is canonically isomorphic to $H^{0}\left(C_{x}, \Omega_{C_{x}}^{1} \otimes \omega_{C_{x}}\right)=H^{0}\left(\pi^{-1}(x), \mathscr{F} \otimes \sigma_{\pi^{-1}{ }_{x}}\right)$, and because $H^{1}\left(C_{x}, \Omega_{C_{x}}^{1} \otimes \omega_{C_{x}}\right)$ $=(0)$. Therefore

$$
K_{\mathscr{M}_{g}^{0}} \equiv\left[\operatorname{ch}\left(\pi_{!} \mathscr{F}\right)\right]_{1} .
$$

We now follow closely the calculations of [13], pp.99-102:

$$
\begin{aligned}
K_{\mathscr{M}_{\mathrm{g}}^{0}} & =\pi_{*}\left(\operatorname{ch}(\mathscr{F}) \cdot T\left(\Omega_{\mathscr{C} / \mathscr{M}}^{1}\right)\right)_{1} \\
& =\pi_{*}\left(\left(1+c_{1}(\mathscr{F})+\frac{c_{1}(\mathscr{F})^{2}}{2}-c_{2}(\mathscr{F})\right) \cdot\left(1-\frac{c_{1}\left(\Omega^{1}\right)}{2}+\frac{c_{1}\left(\Omega^{1}\right)^{2}+c_{2}\left(\Omega^{1}\right)}{12}\right)\right)_{1} \\
& =\pi_{*}\left(\frac{c_{1}(\mathscr{F})^{2}}{2}-c_{2}(\mathscr{F})-\frac{c_{1}(\mathscr{F}) \cdot c_{1}\left(\Omega^{1}\right)}{2}+\frac{c_{1}\left(\Omega^{1}\right)^{2}+c_{2}\left(\Omega^{1}\right)}{12}\right) .
\end{aligned}
$$

Moreover

$$
\Omega_{\mathscr{C} / \mathcal{M}}^{1}=I_{\text {sing }} \cdot \omega_{\mathscr{C} / \mathcal{M}}
$$

where $I_{\text {sing }}$ is the ideal of the singular locus, hence

Therefore

$$
\begin{aligned}
& c_{1}\left(\Omega_{\mathscr{C} / \cdot M}^{1}\right)=c_{1}(\omega), \\
& c_{1}(\mathscr{F})=2 c_{1}(\omega), \\
& c_{2}\left(\Omega_{\mathscr{C} / \cdot M}^{1}\right)=[\operatorname{sing} \mathscr{C}], \\
& c_{2}(\mathscr{F})=[\operatorname{sing} \mathscr{C}] .
\end{aligned}
$$

$(*)$

$$
\begin{aligned}
K_{\mathscr{A}_{\mathrm{g}}} & \equiv \pi_{*}\left(2 c_{1}(\omega)^{2}-[\operatorname{Sing} \mathscr{C}]-c_{1}(\omega)^{2}+\frac{c_{1}(\omega)^{2}+[\operatorname{Sing} \mathscr{C}]}{12}\right) \\
& =\frac{13}{12} \pi_{*}\left(c_{1}(\omega)^{2}\right)-\frac{11}{12} \pi_{*}([\operatorname{Sing} \mathscr{C}]) .
\end{aligned}
$$

On the other hand, let

$$
\lambda=c_{1}\left(\pi_{*} \omega_{\mathscr{B}, \mathcal{M}}\right)
$$

be the so-called Hodge divisor class on $\overline{\mathscr{M}}_{\mathrm{g}}^{0}$. Let

$$
\delta=\pi_{*}([\operatorname{Sing} \mathscr{C}])
$$


be the divisor class of singular curves $\overline{\mathscr{M}}_{\mathrm{g}}^{0}-\mathscr{M}_{\mathrm{g}}$. Then we proved in [13] that

$$
\pi_{*}\left(c_{1}(\omega)^{2}\right)=12 \lambda-\delta .
$$

Combining this with $\left(^{*}\right)$ and recalling that $\operatorname{Pic}\left(\overline{\mathscr{M}}_{\mathrm{g}}^{0}\right)$ is torsion-free ([13], p. 102) we have proven.

Theorem 2. On the smooth variety $\overline{\mathscr{M}}_{g}^{0}, K_{\mathscr{M}_{\mathrm{g}}} \equiv 13 \lambda-2 \delta$.

Does this continue to hold on the bigger open set $\overline{\mathscr{M}}_{g \text {,reg }}$ of all smooth points? The answer depends on how $\lambda$ and $\delta$ are defined on $\overline{\mathscr{M}}_{\text {g,reg }}$. Recall from [13] that to deal with the problems posed by curves with automorphisms, one has 2 approaches:

a) one can introduce the group of line bundles on the moduli functor, i.e., for all flat proper families $\pi: C \rightarrow S$ of stable curves, a line bundle $L(\pi)$ on $S$ and for all Cartesian diagrams

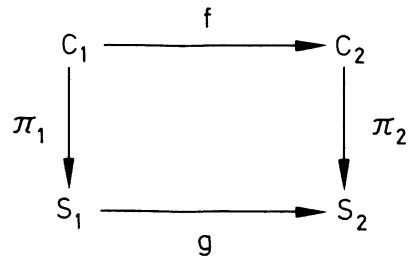

between such families, an isomorphism

$$
L\left(\pi_{1}\right) \cong g^{*} L\left(\pi_{2}\right)
$$

with obvious compatibility requirements. We call this $\operatorname{Pic}_{\text {fun }}\left(\overline{\mathscr{M}}_{\mathrm{g}}\right)$

b) one can introduce the locally closed subscheme $H_{g}$ of a suitable Hilbert scheme parametrizing stable curves in a fixed projective space $\mathbf{P}^{v-1}$. With $H_{g}$, one has

$$
\begin{aligned}
p: Z_{g} \rightarrow & H_{g}: \text { a universal family of curves } \\
& \left(P G L(v) \text { acting on } H_{g}\right) .
\end{aligned}
$$

Then one considers the group $\operatorname{Pic}\left(H_{g}\right)^{P G L(v)}$ of isomorphism classes of line bundles on $H_{\mathrm{g}}$ invariant under $P G L(v)$.

As shown in [13], $\operatorname{Pic}_{\text {fun }}\left(\overline{\mathscr{M}}_{g}\right) \cong \operatorname{Pic}\left(H_{g}\right)^{P G L(v)}$ and $\operatorname{Pic}\left(\overline{\mathscr{M}}_{g}\right)$ itself is a subgroup of these of finite index. Moreover, $\operatorname{Pic}_{\mathrm{fun}}\left(\overline{\mathscr{M}}_{\mathrm{g}}\right)$ is torsion free, so the convenient way to relate these groups is to think of them all as lattices in the same Qvector space

$$
\operatorname{Pic}\left(\overline{\mathscr{M}}_{\mathrm{g}}\right) \otimes \mathbf{Q} \cong \operatorname{Pic}_{\text {fun }}\left(\overline{\mathscr{M}}_{\mathrm{g}}\right) \otimes \mathbf{Q} \cong \operatorname{Pic}\left(H_{g}\right)^{P G L(v)} \otimes \mathbf{Q} .
$$

In the big group $\operatorname{Pic}\left(H_{g}\right)^{P G L(v)}$, we define $\lambda$ to be $c_{1}\left(p_{*} \omega_{Z / H}\right)$ and $\delta$ to be the class of the divisor on $H_{g}$ of singular curves. Now if $L$ is a line bundle on $H_{g}$ with $P G L(v)$ acting equivariantly on it, then for every curve $C$ with automorphism $\phi$ of order $n, \phi$ is induced by $\phi^{\prime} \in P G L(v)$ fixing the point $[C] \in H_{g}$ defined by $C$. Then $\phi^{\prime}$ acts on the fibre $L_{[C]}$ of the line bundle by an $n^{\text {th }}$ root of 1 . If all these roots of 1 are trivial, $L$ descends to a line bundle on $\overline{\mathscr{M}}_{g}=H_{g} / P G L(v)$. For example, if $C$ is 


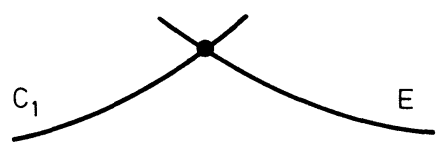

$C_{1}$ of genus $g-1, E$ elliptic, let $g: C \rightarrow C$ be defined by $\left.g\right|_{C_{1}}=$ id., $\left.g\right|_{E}=-1$. For a line bundle $L$ to descend to $\operatorname{Pic}\left(\overline{\mathscr{M}}_{\text {, reg }}\right)$, we only need that this $g$ acts by +1 on $L_{\mathrm{ICl}}$.

As an example, take the Hodge line bundle

$$
L_{\lambda}=\Lambda^{g} p_{*}\left(\omega_{Z_{g} / H_{g}}\right) .
$$

$g$ acts on $\Lambda^{g} H^{0}\left(\omega_{C}\right)$ by -1 , hence $L_{\lambda}$ and the Hodge divisor class $\lambda=c_{1}\left(\Lambda^{g} p_{*} \omega_{Z / H}\right)$ do not descend to $\overline{\mathscr{M}}_{g \text {, reg }}$, but the square of the bundle, or twice the divisor class do. Thus the divisor class $\lambda$ lies in

$$
\frac{1}{2} \operatorname{Pic}\left(\overline{\mathscr{M}}_{\mathrm{g} \text {, reg }}\right) \text {. }
$$

Next, if $L_{\delta_{0}}$ is the line bundle on $H_{g}$ defined by the divisor of irreducible singular curves, and $L_{\delta_{\mathrm{t}}}, 1 \leqq i \leqq\left[\frac{g}{2}\right]$ are the line bundles on $H_{g}$ defined by the divisors of curves with double points separating them into pieces of genus $i$, $g-i$, then the line bundle $L_{\delta}$ of all singular curves breaks up

$$
L_{\delta}=\bigotimes_{i=0}^{\left[\frac{g}{2}\right]} L_{\delta_{i}}
$$

The fibre of $L_{\delta_{1}}$ over a point $[C]$ is

$$
\underset{P}{\otimes} \Lambda^{2}\left(m_{p} / m_{p}^{2}\right)^{*}
$$

where $P$ runs over all double points of $C$ of type $i$ (i.e., non-separating double points for $i=0$, separating with pieces of genus $i, g-i$ for $i \geqq 1$ ). One checks that $L_{\delta_{0}}, L_{\delta_{2}}, \ldots, L_{\delta_{\left[g^{\prime} / 2\right]}}$ all descend to $\overline{\mathscr{M}}_{\mathrm{g} \text {, reg }}$ and are, in fact, the line bundles defined by the divisors $\Delta_{0}, \Delta_{2}, \ldots, \Delta_{\left[\frac{g}{2}\right]} \subset \overline{\mathscr{M}}_{\text {g, reg }}$ of singular curves of various types. But the automorphism $g$ of the curve $C$ with elliptic tail acts by $(-1)$ on the fibre of $L_{\delta_{1}}$ over [C]: hence $L_{\delta_{1}}^{\otimes 2}$ descends to $\overline{\mathscr{M}}_{\mathrm{g} \text {, reg }}$ and is the line bundle defined by the divisor $\Delta_{1} \subset \overline{\mathscr{M}}_{\text {g, reg }}$ of curves with elliptic tails. Thus in terms of divisor classes on $\overline{\mathscr{M}}_{\text {, reg }}$ :

$$
\begin{aligned}
& \delta_{1}=\frac{1}{2} \Delta_{1} ; \quad \delta_{i}=\Delta_{i}, \quad i \neq 1 \\
& \delta=\Delta_{0}+\frac{1}{2} \Delta_{1}+\left(\Delta_{2}+\ldots+\Delta_{\left[\frac{g}{2}\right]}\right) .
\end{aligned}
$$

Note incidentally that $L_{\lambda} \otimes L_{\delta_{1}}$ also descends to $\overline{\mathscr{M}}_{\text {g,reg }}$, hence $\lambda$ $+\frac{1}{2} \Delta_{1} \in \operatorname{Pic}\left(\overline{\mathcal{M}}_{\text {g,reg }}\right)$. On the other hand, the equality of divisor classes on $H_{g}$ :

$$
c_{1}\left(p_{*}\left(\Omega_{Z / H}^{1} \otimes \omega_{Z / H}\right)\right) \equiv 13 \lambda-2 \delta
$$


is proven by exactly the same proof used to prove the same equality in $\operatorname{Pic}\left(\overline{\mathscr{M}}_{\mathrm{g}}^{0}\right)$. The last step is the claim:

Lemma. $K_{\left(\mathscr{M}_{\mathrm{g}, \mathrm{reg}}\right)} \equiv c_{1}\left(p_{*}\left(\Omega_{Z / H}^{1} \otimes \omega_{Z / H}\right)\right)-\delta_{1}$.

Proof. If $f: Z_{g} \rightarrow \overline{\mathscr{M}}_{\mathrm{g}}$ is the canonical map, then on the open set $f^{-1}\left(\overline{\mathscr{M}}_{\mathrm{g}}^{0}\right)$, there is a canonical isomorphism:

$$
\alpha:\left.f^{*}\left(\left.\Omega_{\mathscr{M}_{\mathrm{g}}}^{3 g-3}\right|_{\mathscr{M}_{g}^{0}}\right) \underset{\sim}{\sim} \Lambda^{3 g-3} p_{*}\left(\Omega_{Z / H}^{1} \otimes \omega_{Z / H}\right)\right|_{f^{-1} \mathscr{M}_{g}^{0}}
$$

If $\Delta_{1}^{\prime} \subset H_{g}$ is the divisor of curves with elliptic tails, a priori $\alpha$ has a zero or pole on $\Delta_{1}^{\prime}$ of some order $\ell$, and on all of $f^{-1}\left(\overline{\mathscr{M}}_{\mathrm{g}, \mathrm{reg}}\right)$

$$
f^{*}\left(\Omega_{\mathscr{M}_{\mathrm{g}}}^{3 g-3}\right), \quad \Lambda^{3 \mathrm{~g}-3} p_{*}\left(\Omega_{Z / H}^{1} \otimes \omega_{Z \mid H}\right)\left(\ell \Delta_{1}^{\prime}\right)
$$

are isomorphic. To compute $\ell$, let $C=C_{1} \cup E$ be a curve with elliptic tail such that $C_{1}$ has no automorphisms, $j(E) \neq 0,12^{3}, \infty$. Let $\left(\Delta_{t}^{3 g-3}\right)$ be the base space of the universal deformation of $C$, with coordinates $t_{1}, t_{2}, \ldots, t_{3 g-3}$ where the automorphisms $g: C \rightarrow C$ acts by

$$
g^{*} t_{1}=-t_{1}, \quad g^{*} t_{i}=t_{i}, \quad 2 \leqq i \leqq 3 g-3 .
$$

( $t_{1}=0$ being the locus of singular curves). Then $\overline{\mathscr{M}}_{\mathrm{g}}$ near $[C]$ is just $\Delta_{t}^{3 g-3} /\{e, g\}$, which is the polycylinder $\left(\Delta_{s}^{3 g-3}\right)$ with coordinates $s_{1}=t_{1}^{2}, s_{2}$ $=t_{2}, \ldots, s_{3 g-3}=t_{3 g-3}$. Then $d s_{1} \wedge \ldots \wedge d s_{3 g-3}$ is a local basis of $\Omega_{M_{g}-3}^{3 g}$, while $\Omega_{\Delta_{t}}^{3 g-3}$ is locally the same as $\Lambda^{3 g-3} p_{*}\left(\Omega_{Z / H}^{1} \otimes \omega_{Z / H}\right)$ : thus $d t_{1} \wedge \ldots \wedge d t_{3 g-3}$ is a local basis of the latter. But

$$
d s_{1} \wedge \ldots \wedge d s_{3 g-3}=2 t_{1}\left(d t_{1} \wedge \ldots \wedge d t_{3 g-3}\right)
$$

hence the map from $\Omega_{\mathscr{M}_{g}}^{3 g-3}$ to $\Lambda^{3 g-3} p_{*}\left(\Omega_{Z / H}^{1} \otimes \omega_{Z / H}\right)$ has a simple zero along the locus $t_{1}=0$. This proves that

$$
\left.f^{*}\left(\left.\Omega_{\mathscr{M}_{g}}^{3 g-3}\right|_{\mathscr{M}_{g, \text { reg }}}\right) \cong \Lambda^{3 g-3} p_{*}\left(\Omega_{Z / H}^{1} \otimes \omega_{Z / H}\right)\left(-\Delta_{1}^{\prime}\right)\right|_{f^{-1}\left(\mathcal{M}_{\mathrm{g}, \text { reg }}\right)} .
$$

Taking chern classes, this proves the lemma.

This proves:

Theorem 2 bis. On the smooth variety $\overline{\mathcal{M}}_{\text {, reg }}$,

$$
\begin{aligned}
K_{\mathscr{M}_{\mathrm{g}}} & \equiv 13 \lambda-2 \delta_{0}-3 \delta_{1}-2 \delta_{2}-\ldots-2 \delta_{\left[\frac{8}{2}\right]} \\
& \equiv 13 \lambda-2 \Delta_{0}-\frac{3}{2} \Delta_{1}-2 \Delta_{2}-\ldots-2 \Delta_{\left[\frac{g}{2}\right]}
\end{aligned}
$$

\section{§3. The Class of the Divisor $D_{k}, I$}

For the rest of this article, we assume that the genus $g$ under consideration is odd, and let

$$
g=2 k-1 \text {. }
$$


As in the Introduction, we introduce as the fundamental point of this proof the divisor:

$$
\begin{aligned}
& D_{k} \subset \mathscr{M}_{g}, \\
& D_{k}=\left\{\text { locus of curves } C \text { which are } k \text {-fold covers of } \mathbf{P}^{1}\right\} .
\end{aligned}
$$

Thus if $g=3, k=2, D_{2} \subset \mathscr{M}_{3}$ is the hyperelliptic locus. And if $g=5, k=3, D_{3}$ $\subset \mathscr{M}_{5}$ is the trigonal locus. It is well known that $\operatorname{dim} D_{k}=3 g-4$, hence $D_{k}$ is a divisor. (This can be checked by the usual dimension count, considering the number of branch points for a generic covering $\pi: C \rightarrow \mathbf{P}^{1}$.) The purpose of this section is to prove ${ }^{\star}$ :

Theorem 3. For all $g=2 k-1$, there is a rational number $a_{k}$ such that on $\mathscr{M}_{g}^{0}$ :

$$
\left[D_{k}\right] \equiv a_{k} \lambda .
$$

Corollary. If ${\overline{D_{k}}}_{k} \subset \bar{M}_{g}$ is the closure of $D_{k}$, then there are also integers $n_{k, t}$, $0 \leqq l \leqq[\mathrm{~g} / 2]$ such that on $\overline{\mathscr{M}}_{\mathrm{g}, \mathrm{reg}}$

$$
\left[\bar{D}_{k}\right] \equiv a_{k}\left(\lambda+\frac{1}{2}\left[\Delta_{1}\right]\right)+\sum_{\ell=0}^{\left[\frac{g}{2}\right]} n_{k, \ell}\left[\Delta_{\ell}\right] .
$$

The Corollary follows because $\left[\bar{D}_{k}\right]-a_{k}\left(\lambda+\frac{1}{2}\left[\Delta_{1}\right]\right)$ is an integral divisor class on $\overline{\mathscr{M}}_{\text {g, reg }}$, trivial on the open set $\mathscr{M}_{\text {g, reg }}$, hence is an integral combination of the components $\Delta_{\ell}$ of $\overline{\mathscr{M}}_{\mathrm{g}}-\mathscr{M}_{\mathrm{g}}$.

To prove the theorem, we shall apply Porteous' formula ([2] or [9]) and the Riemann-Roch theorem again. As above, let

$$
\pi: \mathscr{C}_{g}^{0} \rightarrow \mathscr{M}_{g}^{0}
$$

be the universal family of curves. Let

Let

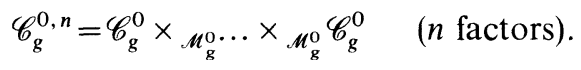

Let

$$
\begin{aligned}
& p_{i}: \mathscr{C}_{g}^{0, n} \rightarrow \mathscr{C}_{g}^{0} \quad \text { be the } i^{\text {th }} \text { projection, } \\
& \pi_{n}: \mathscr{C}_{g}^{0, n} \rightarrow \mathscr{M}_{g}^{0} \quad \text { be the canonical map. }
\end{aligned}
$$

be the $(i, j)^{\text {th }}$ diagonal, and let

$$
\Delta_{i j} \subset \mathscr{C}_{g}^{0, n}
$$

stand for the divisor class

$$
K_{i} \in \operatorname{Pic}\left(\mathscr{C}_{\mathrm{g}}^{0, n}\right)
$$

$$
p_{i}^{*}\left(c_{1}\left(\Omega_{\mathscr{G}_{\mathrm{g}}^{0} / \mathcal{M}_{\mathrm{g}}}^{1}\right)\right)
$$

We are interested in the Zariski-closed subset $Z \subset \mathscr{C}_{\mathrm{g}}^{0, k}$ defined by:

$$
Z=\left\{\left(P_{1}, \ldots, P_{k}\right) \in \mathscr{C}_{g}^{0, k} \mid h^{0}\left({ }_{{ }_{C}}\left(\sum_{1}^{k} P_{i}\right)\right) \geqq 2\right\} .
$$

$\star \quad$ See note at end of article. 
$D_{k}$ is, by definition, $\pi_{k}(Z) \subset \mathscr{M}_{g}$. For simplicity in what follows, we drop the $g$ and 0 in $\mathscr{C}_{\mathrm{g}}^{0, k}$. To compute $Z$, consider

and

$$
R^{0} p_{*} \sigma_{\mathscr{G} k+1}\left(\sum_{j=1}^{k} \Delta_{j, k+1}\right)
$$

where

$$
R^{1} p_{*} o_{\mathscr{C}^{k+1}}\left(\sum_{j=1}^{k} \Delta_{j, k+1}\right)
$$

$$
p: \mathscr{C}^{k+1} \rightarrow \mathscr{C}^{k}
$$

is the projection onto the $1^{\text {st }} k$ factors. Since $\mathscr{C}^{k}$ is an integral scheme and for generic $P_{1}, \ldots, P_{k}, H^{0}\left(o_{C}\left(\sum_{1}^{k} P_{i}\right)\right)$ consists only in constants, the $0^{\text {th }}$ direct image above is just $o_{\mathscr{C}^{k}}$.

Consider the exact sequence on $\mathscr{C}_{g}^{0, k+1}$ :

$$
0 \rightarrow o_{\mathscr{C}^{k+1}} \rightarrow o_{\mathscr{C}^{k}+1}\left(\sum \Delta_{j, k+1}\right) \rightarrow o_{\mathscr{G}^{k+1}}\left(\sum \Delta_{j, k+1}\right) / o_{\mathscr{C}^{k+1}} \rightarrow 0 .
$$

Taking higher direct images, we find:

$$
0 \rightarrow \underbrace{p_{*} \sigma_{\mathscr{C} k+1}\left(\sum \Delta_{j, k+1}\right) / \sigma_{\mathscr{G} k+1}}_{\text {loc. free } r k k} \stackrel{\alpha}{\longrightarrow} \underbrace{R^{1} p_{*} \sigma_{\mathscr{G} k+1}}_{\text {loc. free } r k g} \rightarrow R^{1} p_{*} \sigma_{\mathscr{C}^{k+1}}\left(\sum \Delta_{j, k+1}\right) \rightarrow 0
$$

Since the 2 locally free sheaves, after $\otimes \mathbf{k}(z), z \in \mathscr{C}^{\mathbf{k}}$, give

$$
H^{0}\left(\sigma_{C}\left(\sum P_{j}\right) / \sigma_{C}\right) \stackrel{\alpha(z)}{\longrightarrow} H^{1}\left(\sigma_{C}\right)
$$

with kernel $H^{0}\left(o_{C}\left(\sum P_{j}\right)\right) / \mathbf{C}$, it follows that

$$
Z=\left\{z \in \mathscr{C}^{k} \mid r k \alpha(z) \leqq k-1\right\} .
$$

By Porteous' formula, this implies

$$
[Z]=c_{g-k+1}\left(R^{1} p_{*} o_{\mathscr{G}^{k+1}}\left(\sum \Delta_{j, k+1}\right)\right) .
$$

Here $[Z]$ will be the class of $Z$ counted with some multiplicity: since the result we seek is just that $\left[D_{k}\right] \in \mathbf{Q} \cdot \lambda$, this does not matter ${ }^{\star}$. Since $p_{*} \sigma_{\mathscr{G} k+1}\left(\sum \Delta_{j . k+1}\right)$ $=o_{\mathscr{L} k}$,

$$
[Z]=c_{g-k+1}\left(-p_{!} \wp_{\mathscr{g}^{k}+1}\left(\sum \Delta_{j, k+1}\right)\right) \text {. }
$$

By Grothendieck's Riemann-Roch:

$$
\begin{aligned}
{[Z]=} & \text { polyn. in } c h_{\ell}\left(p_{!} \digamma_{\mathscr{G}^{k+1}}\left(\sum \Delta_{j, k+1}\right)\right) \\
= & \text { polyn. in } p_{*}\left(\operatorname{ch}\left(\digamma_{\mathscr{C}^{k+1}}\left(\sum \Delta_{j, k+1}\right)\right) \cdot T d\left(\Omega_{\mathscr{C}^{k+1} / \mathscr{b}^{k}}^{1}\right)\right)_{\ell} \\
= & \text { polyn. in classes of the form } \\
& p_{*}\left(\text { polyn. in } \sum_{j=1}^{k}\left[\Delta_{j, k+1}\right] \text { and } K_{k+1}\right) .
\end{aligned}
$$

$\star \star$ In fact, it is not hard to see that the multiplicity is one 
To see what this can be, note the easy identities:

$$
\begin{aligned}
{\left[\Delta_{j_{1}, k+1}\right] \cdot\left[\Delta_{j_{2}, k+1}\right] } & =\left[\Delta_{j_{1}, k+1}\right] \cdot p^{*}\left[\Delta_{j_{1}, j_{2}}\right] \\
{\left[\Delta_{j, k+1}\right]^{2} } & =-\left[\Delta_{j, k+1}\right] \cdot p^{*}\left(K_{j}\right) \\
{\left[\Delta_{j, k+1}\right] \cdot K_{k+1} } & =\left[\Delta_{j, k+1}\right] \cdot p^{*}\left(K_{j}\right) \\
p_{*}\left(\left[\Delta_{j, k+1}\right] \cdot p^{*}(a)\right) & =a, \quad \text { any } a \in A\left(\mathscr{C}^{k+1}\right) \\
p_{*}\left(K_{k+1}^{\ell}\right) & =\pi_{k}^{*}\left(\pi_{1_{*}}\left(K_{\mathscr{G}, u}^{\ell}\right)\right) .
\end{aligned}
$$

The second of these is because the self-intersection of $\Delta_{j, k+1}$ is $c_{1}$ of its normal bundle, and the normal bundle is the restriction to $\Delta_{j, k+1}$ of $p_{j}^{*} \Omega_{\mathscr{C} / \mathcal{M}}^{1}$. The last comes from the Cartesian diagram

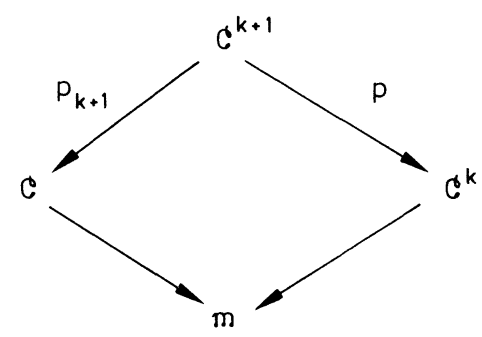

It follows that

$$
[Z]=\text { polyn. in }\left[\Delta_{j_{1}, j_{2}}\right] \text { 's, }\left[K_{j}\right] \text { 's, } \pi_{k}^{*}\left(\pi_{1, *}\left(K_{\mathscr{C} / \mathcal{M}}^{\ell}\right)\right) \text {. }
$$

Now $\left[D_{k}\right]$ is not $\pi_{k, *}([Z])$. Indeed $\pi_{k, *}([Z])=0$ because if a curve has one $\sum P_{i}$ moving in a pencil, it of course has $\infty^{1}$ such cycles. To chop [Z] down in dimension, ask that the first point $P_{1}$ in the cycle $\sum P_{i}$ be a member of a fixed canonical divisor. This gives us

Therefore

$$
(2 g-2)(k-1) !\left[D_{k}\right]=\pi_{k \cdot *}\left([Z] \cdot K_{1}\right) .
$$

$$
\left[D_{k}\right]=\text { polyn. in } \pi_{k, *}\left(\text { polyn. in }\left[\Delta_{j_{1}, j_{2}}\right]^{\prime} \text { s, }\left[K_{j}\right] \text { 's, } \pi_{k}^{*}\left(\pi_{1, *}\left(K^{\ell}\right)\right)\right) .
$$

Now factor $\pi_{k}$ :

$$
\mathscr{C}^{k} \rightarrow \mathscr{C}^{k-1} \rightarrow \mathscr{C}^{k-2} \rightarrow \ldots \rightarrow \mathscr{C} \rightarrow \mathscr{M}
$$

and take images of the above polynomial one at a time. Using the previous identities, it is clear that under each projection

$$
p_{\ell}: \mathscr{C}^{\ell} \rightarrow \mathscr{C}^{\ell-1}
$$

$p_{\ell, *}$ carries any polyn. in $\left[\Delta_{j_{1}, j_{2}}\right]$ 's, $\left[K_{j}\right]^{\prime}$ s and $\pi_{\ell}^{*}\left(\pi_{1, *}\left(K^{\ell}\right)\right)$ into a polynomial of the same type. Finally, projecting to $\mathscr{M}$ itself we deduce

$$
\left[D_{k}\right]=\text { polyn. in } \pi_{1, *}\left(K^{\ell}\right) \text { 's } .
$$

But $\left[D_{k}\right]$ is a divisor, so this just means

$$
\left[D_{k}\right]=\text { multiple of } \pi_{1, *}\left(K^{2}\right) .
$$


By [13], however,

$$
\pi_{1, *}\left(K^{2}\right)=12 \lambda . \quad \text { Q.E.D. }
$$

It should be pointed out that this argument establishes more generally that for any integers $k, a_{1}, \ldots, a_{n} \geqq 2 g^{n \geqq 1}$ and $g=2 k+n-\Sigma a_{i}$, the divisor $\bar{D}_{a . k}$ in $\mathscr{M}_{\mathrm{g}}$ defined as the closure of

$$
D_{\underline{a}, k}=\left\{\begin{array}{c|c}
C \in \mathscr{M}_{g} & \begin{array}{c}
\exists \text { divisor } D=\sum a_{i} p_{i}+D_{0} \in C_{k} \\
h^{0}\left(O_{C}(D)\right) \geqq 2
\end{array}
\end{array}\right\}
$$

is similarly linearly equivalent to a linear combination of $\lambda$ and the $\left[\Delta_{i}\right]$. Indeed, Diaz has used this set-up to explicitly calculate the coefficient of $\lambda$ in the expression for the class of the divisor $D_{g-1, g-1}$ of curves with a Weierstrass point $p$ with $h^{0}\left(C, O_{C}((g-1) p)\right) \geqq 2$; he finds that in $\mathscr{M}_{\mathrm{g}}^{0}$, this coefficient equals $g^{2}(3 g-1)(g-1) / 2 .^{\star \star \star}$

\section{§4. Parametrization of $\bar{D}_{k}$}

It is not obvious which stable curves are in the closure of $D_{k}$, especially which reducible stable curves. To have a way to enumerate the points of $\bar{D}_{k}$ as well as to determine the tangent plane to smooth branches of $\bar{D}_{k}$, we introduce a new moduli space, which will be a compactification of what is usually called the Hurwitz scheme. Recall that the Hurwitz scheme in its simplest form parametrizes the family of $k$-sheeted coverings of $\mathbf{P}^{1}$ with $b$ ordinary branch points:

$$
H_{k, b}=\left\{\begin{array}{c}
\text { moduli space of the data } \\
\pi: C \rightarrow \mathbf{P}^{1} \text { of degree } k \\
P_{1}, \ldots, P_{b} \in \mathbf{P}^{1} \text { distinct } \\
C \text { smooth curve, } \pi \text { with one ordinary branch } \\
\text { point over each } P_{i} \text {, otherwise unbranched }
\end{array}\right\} .
$$

(It is usual to treat the $\left\{P_{i}\right\}$ as a cycle $\sum P_{i}$, but for our purposes we wish to order them.) By the usual theory (see [6]), $H_{k, b}$ is itself a finite étale cover of the space of sequences $\left\{P_{i}\right\}$ :

$$
H_{k, b} \rightarrow\left[\left(\mathbf{P}^{1}\right)^{b}-\bigcup_{i<j} \Delta_{i j}\right] / P G L(2) \cong\left(\mathbf{P}^{1}\right)^{b-3}-S
$$

where $S=\left(\bigcup_{i<j} \Delta_{i j}\right) \cup \bigcup_{i} p_{i}^{-1}(\{0,1, \infty\})$ (normalizing $\left.P_{b-2}=0, P_{b-1}=1, P_{b}=\infty\right)$. By Hurwitz's formula, the genus $g$ of $C$ is given by

$$
2 g-2=-2 k+b
$$

and we have a diagram

$\star \star \star$ See note at end of article 


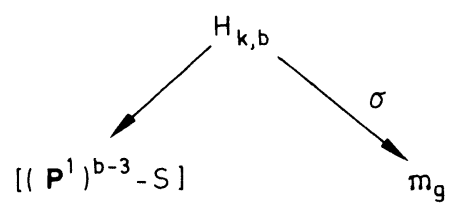

In particular, if $g=2 k-1, D_{k}=\sigma\left(H_{k, b}\right)$. We want to compactify $H_{k, b}$ in such a way that $\sigma$ extends to a morphism

$$
\sigma: \bar{H}_{k, b} \rightarrow \bar{M}_{g}
$$

We do this by means of the theory of F. Knudsen [10] of $b$-pointed curves with fine structure. Knudsen has introduced a smooth projective compactification

$$
P_{b} \supset\left[\left(\mathbf{P}^{1}\right)^{b-3}-S\right]
$$

which as a moduli space can be described as follows:

i) a stable $b$-pointed curve is a reduced, connected curve $C$ with at most ordinary double points, plus $b$ smooth distinct points $P_{1}, \ldots, P_{b} \in C$ such that every smooth rational component $E$ of $C$ contains at least 3 points which are either $P_{i}^{\prime}$ 's or double points of $C$

ii) $P_{b}=\left\{\begin{array}{l}\text { set of } b \text {-pointed stable curves } C \text { with } p_{a}(C)=0 \\ \text { up to isomorphism }\end{array}\right\}$

iii) the open dense set $\left(\mathbf{P}^{1}\right)^{b-3}-S$ is the set of $\left(C, P_{1}, \ldots, P_{b}\right)$ in $P_{b}$ where $C$ is irreducible.

In fact, Knudsen describes $P_{b}$ as an explicit blow-up of $\left(\mathbf{P}^{1}\right)^{b-3}$ along an ideal sheaf with support $S$.

We next describe a functor that will be coarsely represented by the soughtfor scheme $\bar{H}_{k, b}$ :

Definition. $\overline{\mathscr{H}}_{k, b}$ is the functor which associates to a scheme $S$ the set of isomorphism classes of the following data:

i) a stable $b$-pointed curve $\left(D ; P_{1}, \ldots, P_{b}\right)$ of genus 0 over $S$,

ii) an admissible covering $\pi: C \rightarrow D$.

By an admissible covering, we mean that $C / S$ itself is a proper flat family of reduced connected curves with at most ordinary double points, that $\pi$ is étale except at unique smooth points $Q_{i}: S \rightarrow C$, one over each $P_{i}: S \rightarrow D$, where it has ordinary branching (i.e., $\pi: C \rightarrow D$ is analytically just $u=x^{2}, x$ coordinate on $C$ over $S, u$ coordinate on $D$ over $S$ ), and except over the double points of $D / S$. For each $s \in S$ and each point $x$ of $C_{s}$ over a double point $y$ of a fibre $D_{s}$, $C_{s}$ has an ordinary double point and locally $C, D$ and $\pi$ are described by:

$$
\begin{aligned}
& C: x y=a, \quad a \in \hat{o}_{s}, x, y \text { generate } \hat{m}_{x, C} \\
& D: u v=a^{p}, \quad u, v \text { generate } \hat{m}_{y, D} \\
& \pi: u=x^{p}, v=y^{p}
\end{aligned}
$$

for some $p$. (This definition generalizes Beauville's admissible double coverings, used to compactify the space of double coverings of a curve of genus $g$.) 
Theorem 4. The functor $\overline{\mathscr{H}}_{k, b}$ is coarsely represented by a scheme $\bar{H}_{k, b}$ finite over $P_{b}$, i.e., there is a morphism

$$
\overline{\mathscr{H}}_{k, b} \rightarrow \bar{H}_{k, b}
$$

bijective on C-valued points, which is universal for morphisms from $\overline{\mathscr{H}}_{k, b}$ to schemes. Moreover, $\bar{H}_{k, b}$ represents $\overline{\mathscr{H}}_{k, b}$ on the open set of coverings $\pi: \stackrel{C \rightarrow D}{\rightarrow}$ such that $C$ has no automorphism $\alpha: C \rightarrow C$ with $\pi \circ \alpha=\pi$ except the identity.

Proof. The functor $\overline{\mathscr{H}}_{k, b}$ and hence the scheme $\bar{H}_{k, b}$, if it exists, both lie over $P_{b}$, so the problem is local over $P_{b}$. Therefore we may cover $P_{b}$ by suitable open sets and make the construction separately over each. Take a point $\left[D_{0}\right] \in P_{b}$. It may happen that every component of $D_{0}$ has at least one of the $b$-points $P_{i}$ on it. But if not, choose further points $P_{b+1}, \ldots, P_{c} \in D_{0}$ so we have one $P_{i}$ in each component and in some neighborhood $U \subset P_{b}$ of $\left[D_{0}\right]$, choose smooth disjoint sections of the universal curve $\mathscr{D} \rightarrow P_{b}$ through these points. (This is possible because there are birational morphisms $\mathscr{D} \rightarrow \mathbf{P}^{1} \times P_{b}$ which over $\left[D_{0}\right]$ take any one of the components of $\mathscr{D}$ isomorphically to $\mathbf{P}^{1}$, collapsing the rest to points; and $\mathbf{P}^{1} \times P_{b}$ has a section through any point of $\mathbf{P}^{1} \times\left[D_{0}\right]-$ see Knudsen [10].) Choose $U$ small enough so that for all $[D] \in U$, the sections $P_{1}, \ldots, P_{c}$ meet every component of $D$. Moreover, choose $U$ 's well enough so we may find a local coordinate $t_{i}$ on the fibres of $\mathscr{D}$ over $U, t_{i}=0$ on the section $P_{i}: U \rightarrow \mathscr{D}$ to $1^{\text {st }}$ order. Then for all $[D] \in U$, if $\pi: C \rightarrow D$ is any admissible cover, ${ }^{{ }_{C}}\left(\sum_{1}^{c} \pi^{-1} P_{i}\right)$ is ample on $C$. For some $n,{ }^{\circ} C\left(n \sum_{1}^{c} \pi^{-1} P_{i}\right)$ is very ample for all $\pi: C \rightarrow D$.

Next define a "rigidified" version of $\overline{\mathscr{H}}_{k, b}$ over $U$ :

$$
\begin{gathered}
\overline{\mathscr{H}}_{k, b}^{U}=\text { functor of families of admissible coverings } \\
\pi: C \rightarrow D,[D] \in U \text { plus orderings of } \pi^{-1}\left(P_{i}\right): \\
P_{1,1}, \ldots, P_{1, k-1} \in C, \quad \text { the points over } P_{1} \in D, P_{1,1} \text { ramified, } \\
\ldots \\
\ldots \\
P_{b, 1}, \ldots, P_{b, k-1} \in C, \text { the points over } P_{b} \in D, P_{b, 1} \text { ramified, } \\
P_{b+1,1}, \ldots, P_{b+1, k} \in C, \text { the points over } P_{b+1} \in D \\
\ldots \\
P_{c, 1}, \ldots, P_{c, k} \in C, \quad \text { the points over } P_{c} \in D
\end{gathered}
$$

plus a choice of square root

$$
\sqrt{t_{i}} \in \hat{o}_{P_{1,1}, C}, \quad 1 \leqq i \leqq b .
$$

Note that by changing these choices in the obvious way, the finite group

$$
G=\left(\mathbf{Z} / 2 \mathbf{Z} \times \Sigma_{k-2}\right)^{b} \times\left(\sum_{k}\right)^{c-b}
$$

acts on $\overline{\mathscr{H}}_{k, b}^{U}$, where $\Sigma_{\ell}=$ permutations of $\ell$ letters. Via this action $\overline{\mathscr{H}}_{k, b}(\operatorname{Spec} \mathbf{C})$ $\supset \overline{\mathscr{H}}_{k, b}^{U}(\operatorname{Spec} \mathrm{C}) / G$. We shall show that $\overline{\mathscr{H}}_{k, b}^{U}$ is representable. To do this, note that the projection

$$
\pi: C \rightarrow D
$$


and the choice of $t_{i} \in m_{P_{t} . D}, t \notin m_{P_{1} . D}^{2}$, defines isomorphisms:

or

$$
\hat{o}_{P_{l, 1}, C} \stackrel{\approx}{\longrightarrow} \hat{o}_{P_{i}, D}\left[\sqrt{t_{i}}\right] \stackrel{\approx}{\longrightarrow} \mathbf{C}\left[\left[\sqrt{t_{i}}\right]\right] \quad \text { if } 1 \leqq i \leqq b
$$

$$
\hat{o}_{P_{l},,} \stackrel{\approx}{\longrightarrow} \hat{o}_{P_{l}, D} \stackrel{\approx}{\longrightarrow} \mathbf{C}\left[\left[t_{i}\right]\right] \quad \text { if } j>1 \text { or } i>b .
$$

Therefore, if $N$ is large enough, we get an injection:

$$
\begin{aligned}
H^{0}\left(C, o_{C}\left(n \sum_{1}^{c} \pi^{-1} P_{i}\right)\right) & \longrightarrow \mathbf{C}^{k(N+1) c} \\
f & \longrightarrow\left(\ldots, a_{i j k}, \ldots\right)
\end{aligned}
$$

if, near $P_{i j}, f$ is expanded:

or

$$
f=\sum_{k=0}^{N} a_{i j k} t_{i}^{-n+k}+\ldots
$$

$$
f=\sum_{k=0}^{2 N+1} a_{i 1 k} t_{i}^{-n+k / 2}+\ldots \quad \text { if } j=1, i \leqq b .
$$

In other words, for each admissible $\pi: C \rightarrow D$, we get canonically both a subspace $V \subset \mathbf{C}^{k(N+1)^{c}}$ and an embedding

$$
C \subset \mathbf{P}(V) \text {. }
$$

The dimension of $V$ is given by the Riemann-Roch theorem on $C$ as $n k c-g$ +1 . Reversing this process, let $G$ be the Grassmannian of $(n k c-g+1)$ dimensional subspaces of $\mathbf{C}^{k(N+1) c}$, let $\mathscr{V} \rightarrow G$ be the universal vector bundle, and let $H$ be the Hilbert scheme of $\mathbf{P}(\mathscr{V})$ over $G$ of curves in $\mathbf{P}(\mathscr{V})$ with Hilbert polynomial $n k c X-g+1$. By the given ordering of the coordinates in $\mathbf{C}^{k(N+1) c}$, we get maps:

$$
\lambda_{i j}: V \subset \mathbf{C}^{k(N+1) c} \underset{\substack{\text { via } \\ \text { coord } \\ a_{1, k}}}{\longrightarrow}\left\{\sum_{k=0}^{N} a_{k} t_{i}^{-n+k}\right\} \underset{\substack{\text { mult. by } \\ t_{i}^{n}}}{\longrightarrow} \mathbf{C}\left[\left[t_{i}\right]\right] /\left(t_{i}^{N+1}\right)
$$

hence over a suitable open subset of $G$, we get canonical embeddings

$$
\phi_{i j}: \operatorname{Spec} \mathbf{C}\left[\left[t_{i}\right]\right] /\left(t_{i}^{N+1}\right) \hookrightarrow \mathbf{P}(V) .
$$

(Replace $t_{i}$ by $\sqrt{t_{i}}$ if $j=1, i \leqq b$.) Let $H_{1} \subset H$ be the locally closed subscheme of points $z$ where $\phi_{i j}$ exist and $\operatorname{Im} \phi_{i j}$ is a subscheme of the curve $C_{z}$ in $\mathbf{P}(\mathscr{V})$ defined by $z$. Let $H_{2} \subset H_{1}$ be the locally closed subset of connected reduced curves $C_{z}$ with at most ordinary double points with $\operatorname{Im} \phi_{i j}$ being disjoint smooth points $P_{i, j}$ of $C_{z}$, with $C_{z}$ embedded by a complete linear system and with ${ }^{o} C_{z}(1) \cong{ }^{o} C_{z}\left(2 n \sum_{1}^{b} P_{i 1}+n \sum_{\text {rest }} P_{i j}\right)$. Let $H_{3} \rightarrow H_{2} \times U$ be the Hilbert scheme representing morphisms $\pi: C_{z} \rightarrow D,[D] \in U$, and let $H_{4} \subset H_{3}$ be the locally closed set where $\pi$ is finite of degree $k$ over each component and 


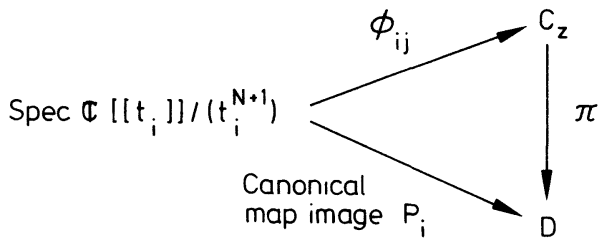

commutes (modify this for $\phi_{i 1}, \ldots, \phi_{i b}$ in the obvious way). Then $H_{4}$ will represent $\overline{\mathscr{H}}_{\boldsymbol{k}, \boldsymbol{b}}^{U}$ !

It follows that $H_{4} / G$ coarsely represents the open subfunctor of $\overline{\mathscr{H}}_{k, b}$ of $\pi$ : $C \rightarrow D$ with $[D] \in U$, and represents it where $G$ acts freely. But a fixed point of $g \in G$ means a covering $\pi: C \rightarrow D$ such that there is an isomorphism:

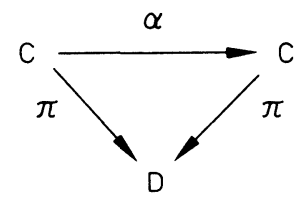

which permutes the finite sets $\pi^{-1}\left(Q_{i}\right)$ and/or acts by $\sqrt{t_{i}} \rightarrow-\sqrt{t_{i}}$ at the ramified points $P_{i, 1}$. Glueing together the schemes $H_{4} / G$ over various open sets $U \subset P_{b}$, we construct $\bar{H}_{k, b}$.

It is clear that the morphism $\bar{H}_{k, b} \rightarrow P_{b}$ is finite to one. To see that it is finite, we check that the functor $\overline{\mathscr{H}}_{k, b}$ satisfies the weak valuative criterion for properness, hence $\bar{H}_{k, b}$ is proper over $\mathbf{C}$. This means that given

$$
\pi: C \rightarrow D
$$

over the field $\mathbf{C}((t))$, then taking a suitable root $t^{1 / n}$ of $t, \pi$ extends to a family of admissible coverings:

$$
\pi_{n}: \mathscr{C}_{n} \rightarrow \mathscr{D}_{n}
$$

over the ring $\mathbf{C}\left[\left[t^{1 / n}\right]\right]$. In fact, by Knudsen's results, $P_{b}$ is complete, hence $D \subset \mathscr{D}_{1}, \mathscr{D}_{1}$ a $b$-pointed stable curve over $\mathbf{C}[[t]]$. Let $\mathscr{C}_{1}$ be the partial normalization of $\mathscr{D}_{1}$ in the fraction field of $C$ defined by

$$
\sigma_{\mathscr{C}_{1}}=\left(\text { functions integral over } o_{\mathscr{D}} \text {, generically in } \sigma_{C}\right) \text {. }
$$

$\mathscr{C}_{1}$ may be ramified over one of the components of the fibre $\left(\mathscr{D}_{1}\right)_{0}$ over $t=0$. But replacing $t$ by $t^{1 / n}$, by Abhyankar's lemma, this no longer happens. This gives us $\mathscr{C}_{n} \rightarrow \mathscr{D}_{n}$ which, by the purity of the branch locus, is ramified only over the sections $P_{i}:$ Spec $k[[t]] \rightarrow \mathscr{D}_{n}$ and the singular points of the fibres of $\mathscr{D}_{n}$. I claim that $\mathscr{C}_{n}$ must be an admissible covering. There are 2 points to check: what happens over an ordinary double point of $\left(\mathscr{D}_{n}\right)_{0}$ which lifts to the generic fibre $\left(\mathscr{D}_{n}\right)_{\eta}$, and what happens over those that don't. In the first case, $\mathscr{D}_{n}$ has the local equation

$$
u \cdot v=0
$$

and $\mathscr{C}_{n}$ is a covering of the smooth branch $u=0$ plus a covering of the smooth branch $v=0$. So if $\left(\mathscr{C}_{n}\right)_{\eta}$ has $p$-fold branching on the curve $u=v=0$ on the surface 
$u=0$ and on the curve $u=v=0$ on the surface $v=0, \mathscr{C}_{n}$ must do the same. In the second case, $\mathscr{D}_{n}$ has the local equation

$$
u \cdot v=s^{m} \quad\left(s=t^{1 / n}\right)
$$

for some $m$, i.e., $\mathscr{D}_{n}$ has a singularity of type $A_{m-1}$. The universal cover of $\mathscr{D}_{n}$, ramified only at the origin, is then

$$
x^{\prime} \cdot y^{\prime}=s, \quad x^{\prime}=u^{1 / m}, \quad y^{\prime}=v^{1 / m} .
$$

Thus $\mathscr{C}_{n}$ must be given by

$$
x \cdot y=x^{k}, \quad x^{\ell}=u, \quad y^{\ell}=v
$$

for some factorization $k \ell=m$, and this is admissible. Q.E.D.

The scheme $\bar{H}_{k, b}$ maps on the one hand to $P_{b}$ by considering only $D$ in the cover $\pi: C \rightarrow D$, and on the other hand to $\bar{M}_{\mathrm{g}}$ by considering only $C$ :

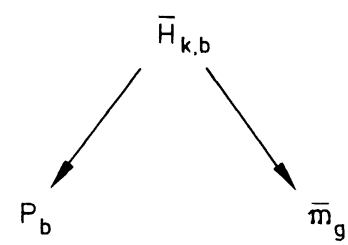

( $C$ is not necessarily stable, but one may contract unnecessary smooth rational components with only 1 or 2 double points, obtaining a stable curve - see Knudsen [10].) If $g=2 k-1$, it is clear that the divisor $\bar{D}_{k} \subset \overline{\mathscr{M}}_{\mathrm{g}}$ is the image of the morphism $\bar{H}_{k, b} \rightarrow \bar{M}_{g}$.

We want to discuss the local structure of $\bar{H}_{k, b}$. More precisely, what family pro-represents the infinitesimal deformations of a particular admissible covering $\pi: C \rightarrow D$ ? Let $\left\{Q_{i}\right\}_{1 \leqq i \leqq d}$ be the double points of $D$ and let $\pi^{-1}\left(Q_{i}\right)$ $=\left\{Q_{i, 1}, \ldots, Q_{i, r(i)}\right\}$. Say the covering $\pi$ is ramified with order $p(i, j)$ at $Q_{i, j}$. Now let

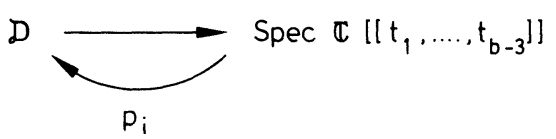

be the universal deformation of the $b$-pointed curve $D$. If $u_{i}, v_{i}$ are coordinates at $Q_{i} \in D$ so that $D$ is given by $u_{i} v_{i}=0$, then if the $t_{i}$ are suitably chosen, we may assume that $\mathscr{D}$ is given locally near $Q_{i}$ by the equation

$$
u_{i} v_{i}=t_{i} .
$$

In order to lift the covering $\pi: C \rightarrow D$ to a covering $\pi: \mathscr{C} \rightarrow \mathscr{D}$, Grothendieck's theory ([8] or [14]) tells us that we must merely give $\mathscr{C}$ locally near all nonétale points of $\pi: C \rightarrow D$. Over each of the $b$ sections $P_{i}$ of $\mathscr{D} / \mathbf{C}\left[\left[t_{1}, \ldots, t_{b-3}\right]\right]$, let $s_{i}$ be a function on $\mathscr{D}$ vanishing to $1^{\text {st }}$ order on the image of $P_{i}$. Then near 
$P_{i}$, the covering $\pi: \mathscr{C} \rightarrow \mathscr{D}$ is given by adjoining $\sqrt{s_{i}}$. At each $Q_{i j}$, however, we must define $\mathscr{C}$ by

and $\pi$ by

$$
x_{i j} y_{i j}=t_{i j}
$$

$$
u_{i}=x_{i j}^{p(i, j)}, \quad v_{i}=y_{i j}^{p(i, j)} \text {. }
$$

In order to do this, $t_{i}$ must equal $t_{i j}^{p(i, j)}$. The universal way to do this is to take our parameter space to be:

where

$$
\operatorname{Spec} \sigma_{[\pi: C \rightarrow D]},
$$

$$
o_{[\pi: C \rightarrow D]}=\mathbf{C}\left[\left[t_{1}, \ldots, t_{b-3}, t_{1,1}, \ldots, t_{i, j}, \ldots, t_{d, r(d)}\right]\right] /\left(t_{i j}^{p(i, j)}-t_{i}, \text { all } i, j\right) .
$$

Over this base, we have now defined a covering $\pi: \mathscr{C} \rightarrow \mathscr{D}$. The complete local rings of the scheme $\bar{H}_{k, b}$ are, as usual, the rings of invariants of $\sigma_{[\pi: C \rightarrow D]}$ under the finite group of automorphisms $\alpha: C \rightarrow C$ such that $\pi \circ \alpha=\pi$.

Unfortunately, some of the rings $a_{[\pi: C \rightarrow D]}$ are rather messy, esp. not integrally closed. Suppose, however, that for all double points $Q_{i} \in D$, there is at most one $Q_{i j} \in C$ over $Q_{i}$ such that $p(i, j)>1$. Let it be the point $Q_{i, 1}$. Then

$$
\sigma_{[\pi: C \rightarrow D]}=\mathbf{C}\left[\left[t_{1,1}, \ldots, t_{d, 1}, t_{d+1}, \ldots, t_{b-3}\right]\right]
$$

and if, furthermore, $C$ has no automorphisms over $D, \bar{H}_{k, b}$ is smooth at the point defined by $\pi: C \rightarrow D$. For further applications it seems that the normalization of $\bar{H}_{k, b}$ is probably more useful than $\bar{H}_{k, b}$ itself. Note that the integral closure of $o_{[\pi: C \rightarrow D]}$ is a semi-local ring whose local rings are all regular.

As an application of the surjective map

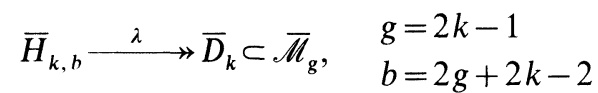

we can describe set-theoretically at least part of $\bar{D}_{k}$ fairly easily. The first part of the following theorem can be proven easily without use of $\bar{H}_{k, b}$, but the second is harder:

Theorem 5. Let $C$ be a stable curve of genus $g=2 k-1,[C] \in \bar{M}_{\mathrm{g}}$ the corresponding point. Then

a) if $C$ is irreducible, $[C] \in \bar{D}_{k}$ if and only if there exists a torsion-free rank 1 sheaf $\mathscr{F}$ on $C$ such that

$$
\begin{aligned}
h^{0}(\mathscr{F}) & \geqq 2 \\
\chi(\mathscr{F}) & =2-k .
\end{aligned}
$$

b) if $C=C_{1} \cup C_{2}, C_{i}$ irreducible, $C_{1} \cap C_{2}=\{p\}$, then $[C] \in \bar{D}_{k}$ if and only if there are torsion-free rank 1 sheaves $\mathscr{F}_{1}$ on $C_{1}, \mathscr{F}_{2}$ on $C_{2}$ and an integer $\ell$ such that:

$$
\begin{aligned}
& h^{0}\left(\mathscr{F}_{1}\right) \geqq 2, \quad h^{0}\left(\mathscr{F}_{2}\right) \geqq 2, \\
& h^{0}\left(\mathscr{F}_{1}(-\ell p)\right) \geqq 1, \quad h^{0}\left(\mathscr{F}_{2}(-\ell p)\right) \geqq 1, \\
& \chi\left(\mathscr{F}_{1}\right)+\chi\left(\mathscr{F}_{2}\right)=3-k+\ell .
\end{aligned}
$$


Proof. We first prove (a). By the theory of the compactification of $\operatorname{Pic}(C)$, the space of pairs $(C, \mathscr{F}), C$ irreducible, $\mathscr{F}$ torsion-free rank 1 is proper over the moduli space of such $C$ 's, and the conditions in (a) define a closed subset of this space. Therefore the set of irreducible $C$ such that such an $\mathscr{F}$ exists is closed in the space of such $C$ 's. Moreover, if $C$ is smooth and $[C] \in D_{k}$, let $\pi$ : $C \rightarrow \mathbf{P}^{1}$ be the given covering of degree $k, \mathscr{F}=\pi^{*} o(1)$. Then

$$
\chi(\mathscr{F})=\operatorname{deg} \pi-g+1=k-(2 k-1)+1=2-k .
$$

This proves that the $C$ 's satisfying the conditions in (a) contains the set of irreducible $C$ 's such that $[C] \in \bar{D}_{k}$. Conversely, let $C$ satisfy the conditions of (a). We will check that [C] lifts to a point of $\bar{H}_{k, b}$, hence $[C] \in \operatorname{Im}\left(\bar{H}_{k, b} \rightarrow \overline{\mathscr{M}}_{g}\right)$ $=\bar{D}_{k}$. To see this, let $C^{\prime}$ be the desingularization of $C$. We shall embed $C^{\prime}$ in a curve $C^{\prime \prime}$ with ordinary double points so that collapsing rational curves of $C^{\prime \prime}$ we get back to $C$ and we construct at the same time an admissible covering

$$
\pi: C^{\prime \prime} \rightarrow P, \quad P \text { a } b \text {-pointed stable rational curve. }
$$

We start by choosing $s_{1}, s_{2} \in \Gamma(\mathscr{F})$ and letting $\mathscr{F}^{\prime} \subset \mathscr{F}$ be the subsheaf generated by $s_{1}, s_{2}$. Let $A$ be the set of double points of $C$. At each $z \in A, \mathscr{F}_{z}^{\prime}$ is isomorphic either to $a_{z, C}$ or to $m_{z, C}$. Call these subsets $A_{1}, A_{2} \subset A$. Let $C^{*}$ be the partial desingularization of $C$ obtained by separating the branches of $C$ at the $z \in A_{2}$ only. Then there is an invertible sheaf $\mathscr{F}^{*}$ on $C^{*}$ such that if

$$
C^{\prime} \stackrel{f}{\longrightarrow} C^{*} \stackrel{g}{\longrightarrow} C
$$

are canonical maps, $\mathscr{F}^{\prime}=g_{*}\left(\mathscr{F}^{*}\right)$. The function $s_{1} / s_{2}$ defines a morphism

hence

$$
\pi^{*}: C^{*} \rightarrow \mathbf{P}^{1}
$$

Note that

$$
\pi=\pi^{*} \circ f: C^{\prime} \rightarrow \mathbf{P}^{1}
$$

$$
\begin{aligned}
\# A_{2}+\operatorname{deg}(\pi) & =\# A_{2}+\operatorname{deg}\left(\pi^{*}\right) \\
& =\# A_{2}+\operatorname{deg}(\mathscr{F} *) \\
& =\# A_{2}+\chi(\mathscr{F} *)+p_{a}\left(C^{*}\right)-1 \\
& =\chi\left(\mathscr{F}^{\prime}\right)+\left(\# A_{2}+p_{2}\left(C^{*}\right)\right)-1 \\
& =\chi\left(\mathscr{F}^{\prime}\right)+p_{a}(C)-1 \\
& \leqq \chi(\mathscr{F})+2 k-2 \\
& =k .
\end{aligned}
$$

Now $\pi: C^{\prime \prime} \rightarrow P$ will be built up starting from $\pi: C^{\prime} \rightarrow \mathbf{P}^{1}$. Let $S \subset \mathbf{P}^{1}$ be

a) the multiple branch points of $\pi$

b) the images of the points $z_{1, i}, z_{2, i} \in C^{\prime}$ over the double points $z_{i} \in C$

c) the images of $k-\# A_{2}-\operatorname{deg}(\pi)$ further generic points $w_{i} \in C^{\prime}$.

Then $P$ is the original $\mathbf{P}^{1}$, henceforth called $\left(\mathbf{P}^{1}\right)_{0}$, with a "tail" $\mathbf{P}^{1}$ glued at each point of $S$. 
Firstly, if $x \in\left(\mathbf{P}^{1}\right)_{0}$ is a multiple branch point, let $\pi^{-1}(x)=\sum n_{i} y_{i}$. For each $i$, add to $C^{\prime}$ a copy of $\mathbf{P}^{1}$ glued to $C^{\prime}$ at $y_{i}$ and mapped to the tail at $x$ by a generic map $\mathbf{P}^{1} \rightarrow \mathbf{P}^{1}$ of degree $n_{i}$, with $n_{i}$-fold branching at $y_{i}$ :

Examples: $n_{1}=2, n_{2}=1, n_{3}=3$

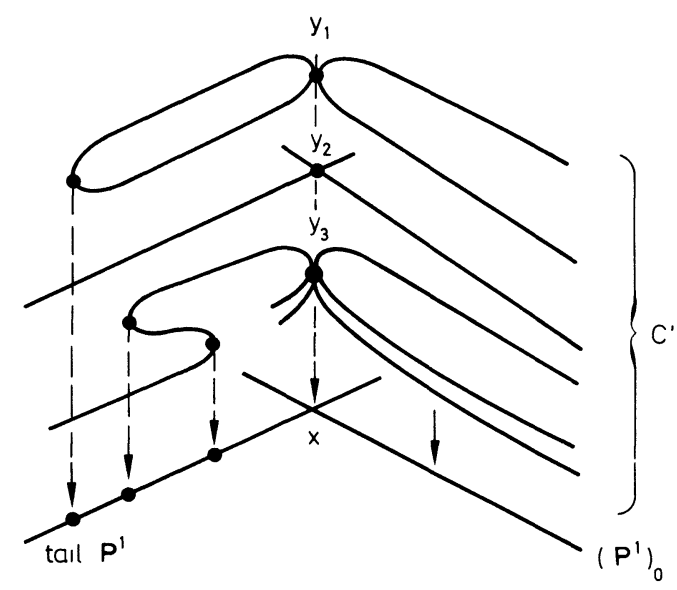

Secondly, if $x \in\left(\mathbf{P}^{1}\right)_{0}$ is the image of $z_{1, i}$ and $z_{2, i}$ (this happens if $z_{i} \in A_{1}$ ), let $\pi$ be ramified to order $\ell_{1}$ at $z_{1, i}, \ell_{2}$ at $z_{2, i}$. Then add one copy of $\mathbf{P}^{1}$ to $C^{\prime}$ meeting $C^{\prime}$ at $z_{1, i}$ and $z_{2, i}$, and lying over the tail at $x$ with degree $\ell_{1}+\ell_{2}$ by a generic $\operatorname{map} \mathbf{P}^{1} \rightarrow \mathbf{P}^{1}$ ramified to order $\ell_{1}$ at $z_{1, i}, \ell_{2}$ at $z_{2, i}$ :

Example: $\ell_{1}=2, \ell_{2}=1$

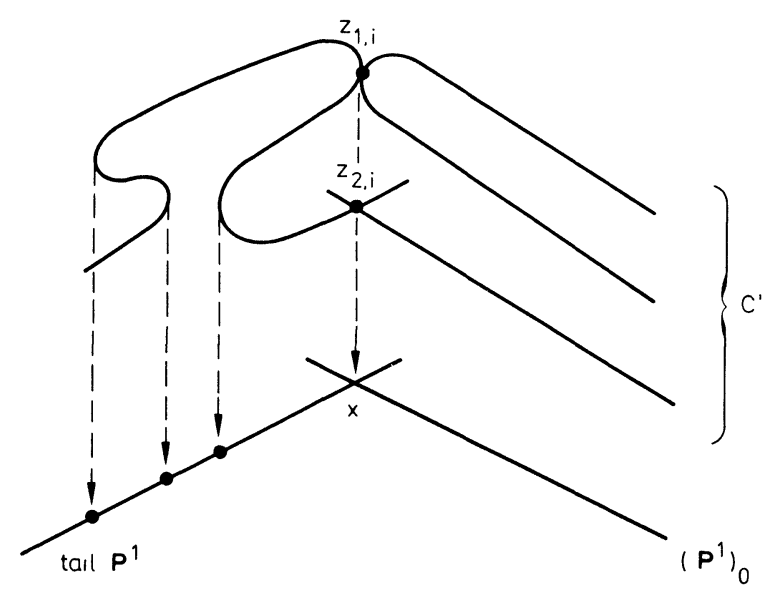

(At other points of $C^{\prime}$ in $\pi^{-1}(x)$, add further $\mathbf{P}^{1}$ 's as in the $1^{\text {st }}$ step.) Thirdly, if $x_{1}, x_{2} \in\left(\mathbf{P}^{1}\right)_{0}, x_{1} \neq x_{2}$ are the images of $z_{1, i}$ and $z_{2, i}$ (this happens if $\left.z_{i} \in A_{2}\right)$, let $\pi$ be ramified to order $\ell_{1}$ at $z_{1, i}, \ell_{2}$ at $z_{2, i}$ again. This time, however, add 3 copies of $\mathbf{P}^{1}$ to $C^{\prime}$, copy $A$ over the tail at $x_{1}$, copy $B$ over the tail at $x_{2}$ and copy $C$ over $\left(\mathbf{P}^{1}\right)_{0}$ as follows: 


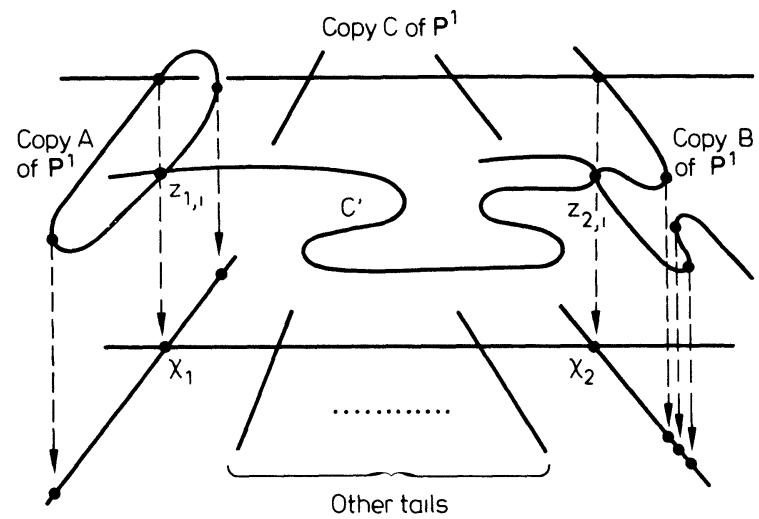

$\ell_{1}=1, \ell_{2}=2$ is illustrated. Note that copy $C$ goes isomorphically to $\left(\mathbf{P}^{1}\right)_{0}$, copy $B$ goes to the tail at $x_{1}$ with degree $\ell_{1}+1$, generic except for $\ell_{1}$-fold ramification at $z_{1, i}$, copy $C$ goes to the tail at $x_{2}$ with degree $\ell_{2}+1$, generic except for $\ell_{2}$-fold ramification at $z_{2, i}$. Both copy $A$ and $B$ meet copy $C$ at their remaining (unramified) point over $x_{1}$ (resp. $x_{2}$ ). Finally, more $\mathbf{P}^{1}$ 's are joined to copy $C$ to cover all the other tails added to $\left(\mathbf{P}^{1}\right)_{0}$. Fourthly, at the $k-\# A_{2}$ $-\operatorname{deg} \pi$ generic points $x$ added to $S$, the following "plumbing fixture" is thrown in to soup up the degree of $\pi$ to $k$ :

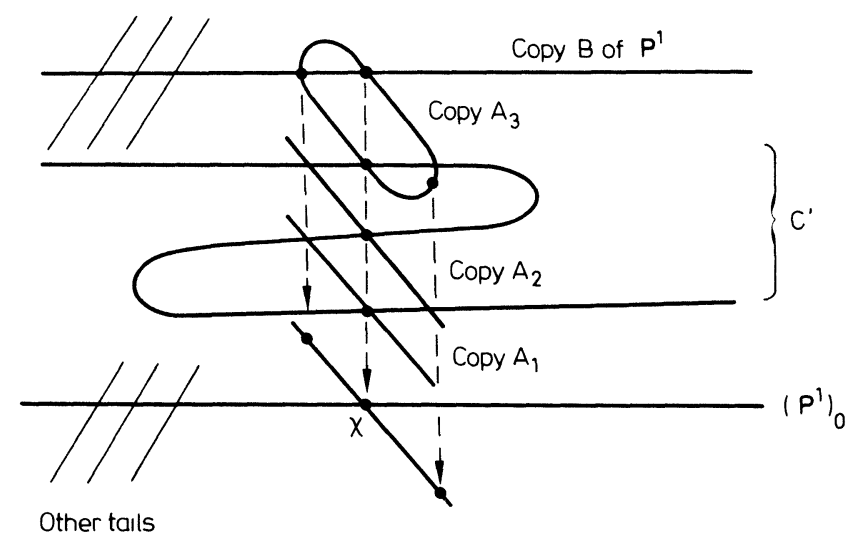

Thus $\operatorname{deg} \pi \mathbf{P}^{1}$ 's are added over the tail at $x$, all but one isomorphic to it, one of degree 2 over it. To the new point over $x$ introduced by this last $\mathbf{P}^{1}$, a copy $B$ of $\mathbf{P}^{1}$ isomorphic to $\left(\mathbf{P}^{1}\right)_{0}$ is joined, and to it further $\mathbf{P}^{1}$ 's over the other tails.

After some reflection, the reader will see that the resulting $C^{\prime \prime}$ is an admissible cover of $P$ of degree $k$, and arithmetic genus $g=2 k-1$, which blows down to the original $C$ when the extra $\mathbf{P}^{1}$ 's are collapsed to points. This completes the proof of part (a).

To check part (b), let $\bar{D}_{k}^{*}$ be the locus of [C]'s satisfying the conditions of parts (a) or (b). We show first that $\bar{D}_{k}^{*}$ is closed in the open set of $\overline{\mathscr{M}}_{g}$ of curves with at most 2 components, meeting at most once, hence in this open set, $\bar{D}_{k}$ 
$\subset \bar{D}_{k}^{*}$, and second that points of $\bar{D}_{k}^{*}$ lift to $\bar{H}_{k, b}$ hence $\bar{D}_{k} \supset \bar{D}_{k}^{*}$. (The latter will use almost the same construction to that we just gave.) It is clear that the locus of curves satisfying (b) is closed in the locus of curves $C_{1} \cup C_{2}, C_{1} \cap C_{2}=\{p\}$. Thus $\bar{D}_{k}^{*}$ is a constructible subset of $\overline{\mathscr{M}}_{g}$. Let $\mathscr{C} / \operatorname{Spec} R$ be a family of stable curves over a valuation ring whose generic member $C^{(\eta)}$ is smooth and in $D_{k}$ and whose special member $C^{(0)}$ is of the form $C_{1}^{(0)} \cup C_{2}^{(0)}, C_{1}^{(0)} \cap C_{2}^{(0)}=\{p\}, C_{i}^{(0)}$ irreducible. Let $\mathscr{F}_{\eta}$ be the invertible sheaf on $C^{(\eta)}$ of degree $k$ with $h^{0}\left(\mathscr{F}_{\eta}\right) \geqq 2$. Then $\mathscr{F}_{\eta}$ extends to a torsion-free, reflexive, rank 1 sheaf on $\mathscr{C}$ in an infinite number of ways parametrized by an integer $k$. If $\mathscr{F} / \mathscr{C}$ is one of these, then $\mathscr{F}\left(-k C_{1}^{(0)}\right) \cong \mathscr{F}\left(+k C_{2}^{(0)}\right)$ are the others. Now the local ring $\hat{o}_{\mathscr{b}, p}$ is isomorphic to

$$
\mathbf{C}[[x, y, t]] /\left(x y-t^{n}\right)
$$

for some $n$, where $t R$ is the maximal ideal of $R$ and $(x, t)=$ ideal of $C_{1}^{(0)},(y, t)$ $=$ ideal of $C_{2}^{(0)}$. Then the group $\operatorname{Pic}\left(\operatorname{Spec} \hat{o}_{\mathcal{E}, p}-\{m\}\right)$ is cyclic of order $n$, being given by the restrictions to the punctured spectrum $\operatorname{Spec} \hat{o}_{\mathscr{G}, p}-\{m\}$ of the ideal sheaves $\left(x, t^{k}\right), 0 \leqq k \leqq n-1$. If $\mathscr{F}$ is isomorphic, as invertible sheaf on Spec $\hat{o}_{\mathscr{Z}, p}$ $-\{m\}$ to $\left(x, t^{k}\right)$, then $\mathscr{F}\left(k C_{1}^{(0)}\right)$ is invertible at $p$. Replacing $\mathscr{F}$ by this, we may as well assume that $\mathscr{F}$ itself is invertible at $p$, hence so are all the sheaves in the sequence

$$
\ldots, \mathscr{F}\left(-n C_{1}^{(0)}\right), \mathscr{F}, \mathscr{F}\left(n C_{1}^{(0)}\right), \mathscr{F}\left(2 n C_{1}^{(0)}\right), \ldots
$$

Restricting $\mathscr{F}$ to $C^{(0)}$, we get a pair of torsion free, rank 1 sheaves $\mathscr{F}_{1}$ on $C_{1}^{(0)}$, $\mathscr{F}_{2}$ on $C_{2}^{(0)}$, invertible at $p$ and "glued" there to give a sheaf on $C^{(0)}$. Restricting the above twists of $\mathscr{F}$, we get the pairs $\mathscr{F}_{1}(k p), \mathscr{F}_{2}(-k p)$, glued to a sheaf $\mathscr{F}_{k}$ on $C^{(0)}$. By upper semi-continuity of $h^{0}$, it follows that $h^{0}\left(\mathscr{F}_{k}\right) \geqq 2$. Fix $k_{1}>k_{2}$ by the hypothesis

$$
\begin{array}{ll}
h^{0}\left(\mathscr{F}_{1}(k p)\right) \geqq 2 & \text { if } k \geqq k_{1}, \\
h^{0}\left(\mathscr{F}_{1}(k p)\right) \geqq 1 & \text { if } k \geqq k_{2} .
\end{array}
$$

Then $h^{0}\left(\mathscr{F}_{k_{1}}\right) \geqq 2$ implies $h^{0}\left(\mathscr{F}_{2}\left(-k_{1} p\right)\right) \geqq 1$ and $h^{0}\left(\mathscr{F}_{k_{2}}\right) \geqq 2$ implies $h^{0}\left(\mathscr{F}_{2}\left(-k_{2} p\right)\right) \geqq 2$. If $\mathscr{F}_{1}^{\prime}=\mathscr{F}_{1}\left(k_{1} p\right), \mathscr{F}_{2}^{\prime}=\mathscr{F}_{2}\left(-k_{2} p\right), \ell=k_{1}-k_{2}$, we see that $\mathscr{\mathscr { F }}_{1}^{\prime}, \mathscr{F}_{2}^{\prime}$ and $\ell$ satisfy the conditions of part (b) so that $\left[\mathrm{C}^{(0)}\right] \in \bar{D}_{k}^{*}$.

For the second part, we start with $C=C_{1} \cup C_{2}, \mathscr{F}_{1}, \mathscr{F}_{2}$, and $\ell$ satisfying (b). Take 2 sections in $\mathscr{F}_{1}$, one a section of $\mathscr{F}_{1}(-\ell p)$, spanning a subsheaf $\mathscr{F}_{1}^{\prime}$, and 2 sections in $\mathscr{F}_{2}$, one a section of $\mathscr{F}_{2}(-\ell p)$, spanning $\mathscr{F}_{2}^{\prime}$ and let their ratios define morphisms of the desingularizations $C_{i}^{(0) \prime}$ of $C_{i}^{(0)}$ :

$$
\begin{aligned}
& \pi_{1}: C_{1}^{(0)^{\prime}} \rightarrow \mathbf{P}^{1} \text { of degree } k_{1}, \text { ramified to order } \ell_{1} \text { at } p \\
& \pi_{2}: C_{2}^{(0) \prime} \rightarrow \mathbf{P}^{1} \text { of degree } k_{2}, \text { ramified to order } \ell_{2} \text { at } p .
\end{aligned}
$$

If it happens that $\ell_{1}=\ell_{2}$, we can join them into an admissible cover by identifying $p$ in $C_{1}^{(0) \prime}, C_{2}^{(0) \prime}$ : 
Example: $\ell_{1}=\ell_{2}=2, n=4$

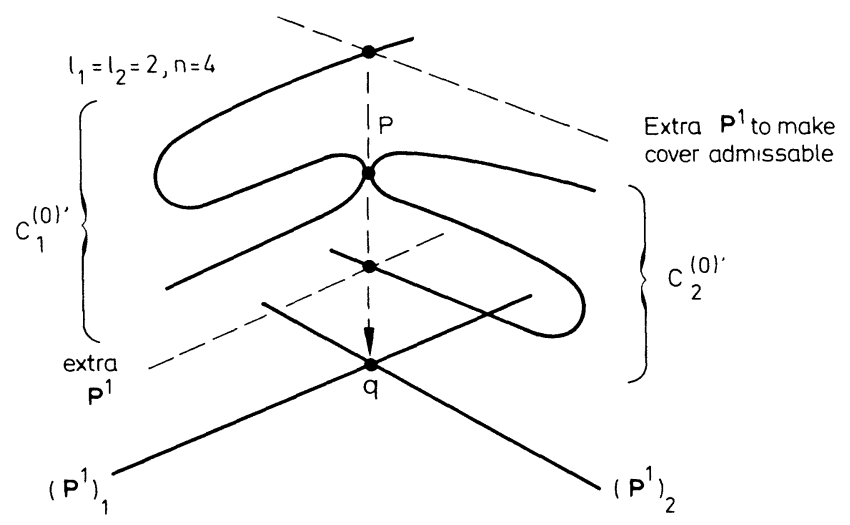

Otherwise, we need an extra $\mathbf{P}^{1}$ to join them:

Example. $\ell_{1}=3, \ell_{2}=2, n=5$

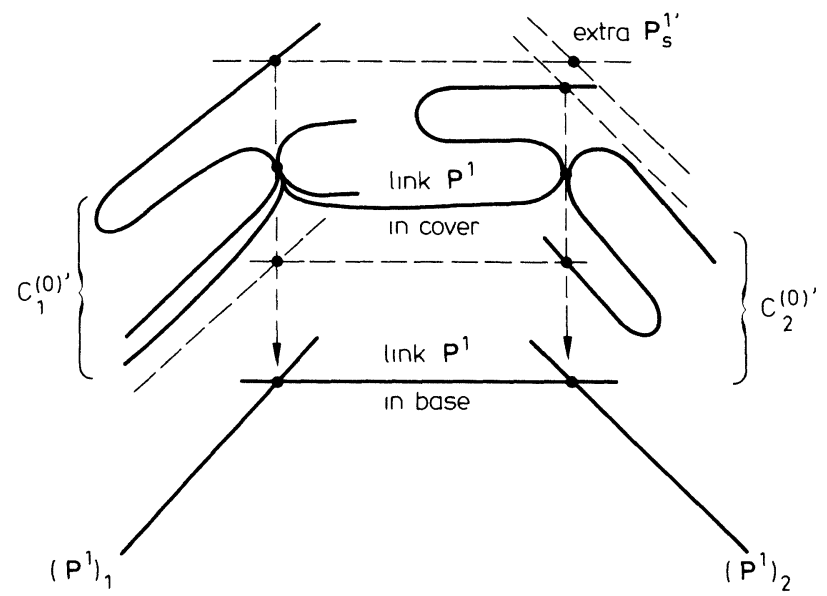

If $\ell_{1}>\ell_{2}$, add a $\mathbf{P}^{1}$ to the cover of degree $\ell_{1}$ over the link $\mathbf{P}^{1}$ in the base ramified to order $\ell_{1}$ at the point $P$ in $C_{1}^{(0), r}$ and to order $\ell_{2}$ at the point $p \in C_{2}^{(0), '}$. At this point, the covering is "filled in", exactly as in the case of an irreducible $C$. To make it admissible, extra $\mathbf{P}^{1}$ 's are needed at multiple branch points of $\pi_{1}$ and $\pi_{2}$. To join the two points of $C_{1}^{(0),{ }^{\prime}}$ (resp. $C_{2}^{\left.(0),{ }^{\prime}\right)}$ over double points of $C_{1}^{(0)}$ (resp. $C_{2}^{(0)}$ ), linking $\mathbf{P}^{1}$ s are needed. Finally the degree of the whole cover must be augmented to $k$. Thus for example, in the case $\ell_{1}=\ell_{2}=2$, $k_{1}=k_{2}=3, k=5$, one would add $\mathbf{P}^{1}$ 's as follows: 
Example:

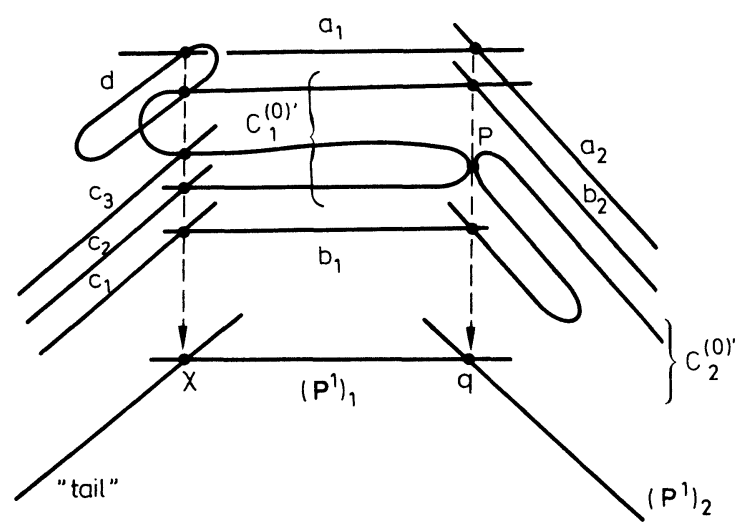

All $a_{i}, b_{i}, c_{i}, d$ are $\mathbf{P}^{1}$ 's. In general, $k_{1}-\ell_{1} \mathbf{P}^{1}$ 's (like $b_{2}$ ) are added to $C_{1}^{(0)}$ to extend its other points over $q$ across $\left(\mathbf{P}^{1}\right)_{2}$, and $k_{2}-\ell_{2} \mathbf{P}^{1}$ 's (like $\left.b_{1}\right)$ are added to $C_{2}^{(0)}$ to extend its other points over $q$ across $\left(\mathbf{P}^{1}\right)_{1}$.

The main point to be checked is that $k$ is large enough to accommodate all these curves. Thus if

$A_{i}=$ set of double points of $C_{i}^{(0)}$ where $\mathscr{F}_{i}^{\prime}$ is not invertible,

to carry out our constructions, we need

$$
\left(k_{1}-\ell_{1}\right)+\left(k_{2}-\ell_{2}\right)+\max \left(\ell_{1}, \ell_{2}\right)+\# A_{1}+\# A_{2}
$$

sheets, so this number must be $\leqq k$. But, in fact, as above,

$$
k_{i}+\# A_{i}=\chi\left(\mathscr{F}_{i}^{\prime}\right)+p_{a}\left(C_{i}^{(0)}\right)-1 .
$$

Moreover, since one of the sections of $\mathscr{F}_{i}$ used to define $\pi_{i}$ vanishes to order at least $\ell$, it follows that

Thus if $\ell_{1} \geqq \ell_{2}$ :

$$
\begin{aligned}
\ell & \leqq \ell_{i}+\ell\left(\mathscr{F}_{i, p} / \mathscr{F}_{i, p}^{\prime}\right) \\
& \leqq \ell_{i}+\chi\left(\mathscr{F}_{i}\right)-\chi\left(\mathscr{F}_{i}^{\prime}\right) .
\end{aligned}
$$

$$
\begin{aligned}
\left(k_{1}-\ell_{1}\right)+\left(k_{2}-\ell_{2}\right)+\max \left(\ell_{1}, \ell_{2}\right)+\# A_{1}+\# A_{2} & =\chi\left(\mathscr{F}_{1}^{\prime}\right)+\chi\left(\mathscr{F}_{2}^{\prime}\right)-\ell_{2}+p_{a}\left(C^{(0)}\right)-2 \\
& \leqq \chi\left(\mathscr{F}_{1}\right)+\chi\left(\mathscr{F}_{2}\right)-\ell+2 k-3 \\
& =(3-k+\ell)-\ell+2 k-3 \\
& =k . \quad \text { Q.E.D. }
\end{aligned}
$$

Working this out more explicitly, we can draw a series of Corollaries from this theorem:

Corollary 1. Let Int $\Delta_{0}$ be the locus of irreducible curves $C$ with one double point p. Writing these curves as the quotient of a curve $C^{\prime}$ of genus $g-1$ by identifying 2 distinct points $p_{1}, p_{2}$, we have:

$$
\text { (Int } \left.\Delta_{0}\right) \cap \bar{D}_{k}=\left\{\left(C^{\prime}, p_{1}, p_{2}\right) \mid \begin{array}{c}
\exists \text { a line bundle } L \text { on } C^{\prime} \text { of degree } k \\
\text { s.t. } h^{0}(L) \geqq 2, h^{0}\left(L\left(-p_{1}-p_{2}\right)\right) \geqq 1
\end{array}\right\} .
$$


The map $\left(C^{\prime}, p_{1}, p_{2}\right) \mapsto C^{\prime}$ carries all components of this intersection onto $\mathscr{M}_{\mathrm{g}-1}$.

Proof. This comes from part (a) of the theorem. The condition above comes from the case where $\mathscr{F}$ is invertible. The other possibility is that $\mathscr{F}_{p} \cong m_{p}$. Such an $\mathscr{F}$ is the image of a line bundle $M$ on $C^{\prime}$ of degree $k-1$ such that $h^{0}(M) \geqq 2$. Let $L=M\left(p_{1}\right)$ and the condition above is satisfied.

Corollary 2. Let Int $\Delta_{0,1}$ be the locus of curves of the type

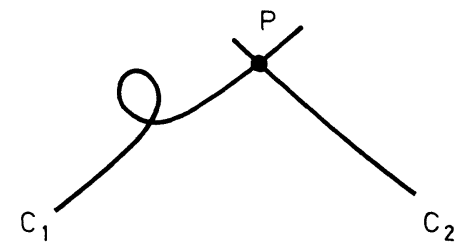

where $C_{2}$ is smooth of genus $g-1$, and $C_{1}$ is $\mathbf{P}^{1}$ with $0, \infty$ identified. Describing these curves by the pair $\left(C_{2}, p\right)$, we have:

$$
\text { (Int } \left.\Delta_{0,1}\right) \cap \bar{D}_{k}=\left\{\left(C_{2}, p\right) \mid \begin{array}{c}
\exists \text { a line bundle } L \text { on } C_{2} \text { of degree } k \\
\text { s.t. } h^{0}(L) \geqq 2, h^{0}(L(-2 p)) \geqq 1
\end{array}\right\} \text {. }
$$

Proof. We use part (b) of the theorem. For $\mathscr{F}_{1}$ to exist, it is necessary and sufficient that $\chi\left(\widetilde{F}_{1}\right), \ell$ satisfy:

$$
\ell=1, \chi\left(\mathscr{F}_{1}\right) \geqq 2 \text { or } \ell \geqq 2, \chi\left(\mathscr{F}_{1}\right) \geqq \ell .
$$

But this means that $\mathscr{F}_{2}$ is a line bundle on $C_{2}$ such that either

or

$$
\operatorname{deg} \mathscr{F}_{2} \leqq k, h^{0}\left(\mathscr{F}_{2}\right) \geqq 2, h^{0}\left(\mathscr{F}_{2}(-\ell p)\right) \geqq 1, \ell \geqq 2
$$

$$
\operatorname{deg} \mathscr{F}_{2} \leqq k-1, h^{0}\left(\mathscr{F}_{2}\right) \geqq 2, h^{0}\left(\mathscr{F}_{2}(-p)\right) \geqq 1 .
$$

All of these imply the existence of $\mathscr{F}_{2}$ such that

$$
\operatorname{deg} \mathscr{F}_{2}=k, h^{0}\left(\mathscr{F}_{2}\right) \geqq 2, h^{0}\left(\mathscr{F}_{2}(-2 p)\right) \geqq 1 .
$$

For the next Corollary, we define:

$\mathscr{M}_{g, 1}=$ moduli space of pairs $(C, p), C$ smooth of genus $g, p \in C$

$S_{k, \ell, \mathrm{g}}=$ subset of $\mathscr{M}_{\mathrm{g}, 1}$ of pairs $C, p$ such that there exists a line bundle $L$ on $C$ of degree $k$ with $h^{0}(L) \geqq 2, h^{0}(L(-\ell p)) \geqq 1$.

Corollary 3. If $1 \leqq g_{1} \leqq k-1$ and $g_{2}=g-g_{1}$, let Int $\Delta_{g_{1}}$ be the locus of curves $C$ $=C_{1} \cup C_{2}$ where $C_{1}, C_{2}$ are smooth with genus $g_{1}, g_{2}$ and $C_{1} \cap C_{2}=\{p\}$. Note that:

Then

$$
\text { Int } \Delta_{g_{1}} \cong \mathscr{M}_{g_{1}, 1} \times \mathscr{M}_{\mathrm{g}_{2}, 1} \text {. }
$$

$$
\begin{gathered}
\left(\text { Int } \Delta_{g_{1}}\right) \cap \bar{D}_{k}=\underset{\frac{g+1}{2} \leqq k_{1} \leqq \min \left(k, g_{1}\right)}{\bigcup}\left(S_{k_{1}, 2 k_{1}-g_{1}, g_{1}} \times \mathscr{M}_{g_{2}, 1}\right) \\
\cup \underset{\frac{g_{2}+1}{2} \leqq k_{2} \leqq \min \left(k, g_{2}\right)}{\bigcup}\left(\mathscr{M}_{g_{1}, 1} \times S_{k_{2}, 2 k_{2}-g_{2}, g_{2}}\right) .
\end{gathered}
$$


Proof. We apply (b). Note that as the $C_{i}$ are smooth, the $\mathscr{F}_{i}$ are line bundles. Let $k_{i}=\operatorname{deg} \mathscr{F}_{i}$. Now $k_{1}, k_{2}$ and $\ell$ satisfy:

$$
\left(k_{1}+1-g_{1}\right)+\left(k_{2}+1-g_{2}\right)=\chi\left(\mathscr{F}_{1}\right)+\chi\left(\mathscr{F}_{2}\right)=3-k+\ell
$$

hence

$$
\begin{aligned}
k_{1}+k_{2} & =1-k+\ell+\left(g_{1}+g_{2}\right) \\
& =k+\ell
\end{aligned}
$$

Now part (b) of Theorem 5 asserts that

\section{But note that:}

$$
\text { (Int } \left.\Delta_{g_{1}}\right) \cap \bar{D}_{k}=\bigcup_{\substack{k_{1}, k_{2}, \ell \\ \text { with } k_{1}+k_{2}=k+\ell}}\left(S_{k_{1}, \ell, g_{1}} \times S_{k_{2}, \ell, g_{2}}\right) \text {. }
$$

i) if $2 k-\ell-1 \geqq g, S_{k, \ell, g}=\mathscr{M}_{\mathrm{g}, 1}$

ii) $S_{k, \ell, g} \subset S_{k+1, \ell+1, g}$.

For (i), see [7]; for (ii), if $L$ has degree $k$ and puts $(C, p)$ in $S_{k, \ell, g}$, then $L(p)$ has degree $k+1$ and puts $(C, p)$ in $S_{k+1, \ell+1, g}$. Now if $k_{1}, k_{2}, \ell$ satisfy

note that:

$$
k_{1}+k_{2}=k+\ell
$$

$$
\text { either } 2 k_{1}-\ell-1 \geqq g_{1} \text {, or } \quad 2 k_{2}-\ell-1 \geqq g_{2} \text {. }
$$

If not,

hence

$$
g_{i} \geqq 2 k_{i}-\ell, \quad i=1,2
$$

$$
2 k-1=g_{1}+g_{2} \geqq 2 k_{1}+2 k_{2}-2 \ell=2 k
$$

a contradiction. But if

Therefore, in the union in $(*)$, one of the 2 factors is always $\mathscr{M}_{g_{1}, 1}$ or $\mathscr{M}_{g_{2}, 1}$.

$$
2 k_{1}-\ell-1>g_{1},
$$

then if we replace $k_{2}$ by $k_{2}+1$ and $\ell$ by $\ell+1$, then

$$
\begin{aligned}
S_{k_{1}, \ell, g_{1}} \times S_{k_{2}, \ell, g_{2}} & =\mathscr{M}_{g_{1}, 1} \times S_{k_{2}, \ell, g_{2}} \\
& \subset \mathscr{M}_{g_{1}, 1} \times S_{k_{2}+1, \ell, g_{2}+1} \\
& =S_{k_{1}, \ell+1, g_{1}} \times S_{k_{2}+1, \ell, g_{2}+1} .
\end{aligned}
$$

Thus in the union in $(*)$, we need only consider the terms with

or

$$
\begin{aligned}
2 k_{1}-\ell-1 & =g_{1}, 2 k_{2}-\ell=g_{2} \\
2 k_{1}-\ell & =g_{1}, 2 k_{2}-\ell-1=g_{2} .
\end{aligned}
$$

This is exactly the Corollary.

Note that in the special cases $g_{1}=1$ or 2 , the Corollary reduces to:

$$
\begin{aligned}
\left(\text { Int } \Delta_{1}\right) \cap \bar{D}_{k} & =\mathscr{M}_{1,1} \times S_{k, 2, g-1}, \\
\left(\text { Int } \Delta_{2}\right) \cap \bar{D}_{k}=\left(S_{2,2,2}\right. & \left.\times \mathscr{M}_{g-2,1}\right) \cup\left(\mathscr{M}_{2,1} \times S_{k, 3, g-2}\right) \\
& \cup\left(\mathscr{M}_{2,1} \times S_{k-1,1, g-2}\right) .
\end{aligned}
$$


Only in these two cases do we have components of Int $\Delta_{g_{1}} \cap \bar{D}_{k}$ consisting entirely of curves with automorphisms: viz. $\mathscr{M}_{1,1} \times S_{k, 2, g-1}$ and $S_{2,2,2}$ $\times \mathscr{M}_{g-2,1}$ (the latter means a curve $C_{1}$ of genus 2 meeting a curve $C_{2}$ of genus $g-2$ at a point $p$, where $p \in C_{1}$ is a Weierstrass point).

Corollary 2 plus the case $g_{1}=1$ combine to say:

\section{Corollary 4.}

$\left(\begin{array}{c}\text { locus of curves } C_{1} \cup C_{2}, C_{2} \text { smooth of genus } g-1 \\ C_{1} \text { smooth or singular of genus } 1, C_{1} \cap C_{2}=\{p\}\end{array}\right) \cap \bar{D}_{k}=\overline{\mathscr{M}}_{1,1} \times S_{k, 2, g-1}$.

\section{§5. Counting Pencils on the Generic Curve}

We need to refine the results in Corollaries 1 and 3 of $\$ 4$ by determining the intersection multiplicities in the intersections $\bar{D}_{k} \cap \operatorname{Int} \Delta_{i}$, and we will need to count the intersections of these cycles with the curves in $\Delta_{i}$ obtained by varying the double points used to get a singular stable curve in $\Delta_{i}$. In order to do both, we need two fundamental results counting pencils on the generic curve of genus $g$, which are essentially but not completely contained in [7]. The results are:

Theorem A. For all $g \geqq 1$ and all $d$ such that

let

$$
\frac{g}{2}+1 \leqq d \leqq g+1
$$

$$
a(d, g)=(2 d-g-1) \frac{g !}{d !(g-d+1) !}
$$

Then for almost all pairs $(C, p), C$ a curve of genus $g, p \in C$, there is a finite number $a(d, g)$ of line bundles $L$ on $C$ of degree $d$ such that

$$
\left.h^{0}(L) \geqq 2, h^{0}(L(-2 d-g-1) p)\right) \geqq 1 .
$$

Moreover, for each $L, h^{0}(L)=2, L$ is generated by $H^{0}(L)$, and $H^{0}(L)$ defines a covering

$$
\pi: C \rightarrow \mathbf{P}^{1}
$$

of degree $d$ with all ordinary branch points except for one $(2 d-g-1)$-fold branch point at $p$, all lying over distinct points of $\mathbf{P}^{1}$.

Theorem B. For all $\mathrm{g} \geqq 1$ and all $d$ such that

$$
\frac{g}{2}+1 \leqq d \leqq g
$$

let

$$
b(d, g)=(2 d-g-1)(2 d-g)(2 d-g+1) \frac{g !}{d !(g-d) !} .
$$


Then for almost all curves $C$ of genus $g$, there is a finite number $b(d, g)$ of pairs $(L, p), L$ a line bundle on $C$ of degree $d$ and points $p \in C$ such that

$$
h^{0}(L) \geqq 2, h^{0}(L(-(2 d-g) p) \geqq 1 .
$$

Moreover, for each $L, h^{0}(L)=2, L$ is generated by $H^{0}(L)$ and $H^{0}(L)$ defines a covering

$$
\pi: C \rightarrow \mathbf{P}^{1}
$$

of degree $d$ with all ordinary branch points and, if $2 d-g \geqq 3$, one $(2 d-g)$-fold branch point, all lying over distinct points of $\mathbf{P}^{1}$.

Proof. Theorem A follows directly from results of [7]. To see this, note first that if $C$ is a general curve, $p \in C$ a general point, then by a naive dimension count there will exist no map

$$
\pi: C \rightarrow \mathbf{P}^{1}
$$

ramified at $p$, whose branch divisor $B$ on $\mathbf{P}^{1}$ is supported on fewer than $3 g+1$ points; or any such map $\pi$ of degree $d-k$ with a $(2 d-g-1-k)$-fold branch point at $p$ for $k>0$; or any such map of degree $d$ with a $(2 d-g-1)$-fold branch point at $p$ such that

$$
h^{0}\left(C, \pi^{*} o_{\mathbf{p}^{1}}(1)\right) \geqq 3 .
$$

Thus, if $(C, p)$ is general, any line bundle $L$ of degree $d$ on $C$ satisfying (*) must satisfy as well the rest of the conditions of the theorem. It then remains only to count the number of points of intersection in the $d^{\text {th }}$ symmetric product $C_{d}$ of $C$ of the cycles

and

$$
C_{d}^{1}=\left\{D \mid h^{0}\left(C, o_{C}(D)\right) \geqq 2\right\}
$$

$$
X_{p}^{2 d-g-1}=\{D \mid D-(2 d-g-1) p \geqq 0\}
$$

This is readily done: the class of the cycle $C_{d}^{1}$ is found to be

$$
c_{d}^{1}=\frac{\theta^{g-d+1}}{(g-d+1) !}-\frac{x \theta^{g-d}}{(g-d) !}
$$

where $x$ is the class of the divisor $X_{p}=\{D \mid D-p \geqq 0\}$ and $\theta$ the pullback from the Jacobian of $C$ of the class of the $\theta$-divisor. By Poincaré's formula, the intersection numbers

$$
\chi^{d-\alpha} \theta^{\alpha}=\frac{g !}{(g-\alpha) !}
$$

and hence the intersection number

$$
\left(c_{d}^{1} \cdot x^{2 d-g-1}\right)=a(d, g)
$$


Finally, in [7] it is shown that if $(C, p)$ is general, then $C_{d}^{1}$ is reduced, and $C_{d}^{1}$ intersects $X_{p}^{2 d-g-1}$ transversely in exactly $a(d, g)$ points, proving Theorem A. ${ }^{3}$

The proof of Theorem B, by contrast, requires a little more care. As in the first case, naive dimension counts show us that, for general $C$, no line bundle $L$ of degree $d$ on $C$ can satisfy $(* *)$ and violate any of the remaining conditions. The problem is thus reduced to computing the intersection number in $C_{d}$ of the cycle $C_{d}^{1}$ with the diagonal

$$
\Delta^{2 d-g}=\{D \mid D-(2 d-g) p \geqq 0 \quad \text { for some } p \in C\}
$$

and showing that this intersection is transverse.

The intersection number is readily computed. The class of $C_{d}^{1}$ is as before; and for $p+q=g-d+1$ the degree of the pullback, via the diagonal map

$$
\begin{aligned}
\Delta: C \times C_{g-d} & \rightarrow C_{d} \\
(p, E) & \mapsto E+(2 d-g) p
\end{aligned}
$$

of the class $x^{p} \cdot \theta^{q}$ is given by

$$
\Delta^{*}\left(x^{p} \theta^{q}\right)=\frac{g !}{(g-p) !}(2 d-g)^{2} p+(2 d-g) q
$$

(cf. $[11,2])$. Combining these yields the intersection number

$$
\left(C_{d}^{1} \cdot \Delta^{2 d-g}\right)=b(d, g) .
$$

It remains to check that for general $C$ this intersection is transverse. To begin with, we may identify the tangent spaces involved as follows:

i) At any point $D \in C_{d}$, we have natural identifications

and

$$
T_{D}\left(C_{d}\right)=\Gamma\left(o_{C}(D) / o_{C}\right)
$$

$$
T_{D}^{*}\left(C_{d}\right)=\Gamma\left(K_{C} / K_{C}(-D)\right)
$$

with the pairing of tangent and cotangent spaces given by the residue.

ii) If $D=m \cdot p+q_{1}+\ldots+q_{d-m}$ with $p, q, \ldots, q_{d-m}$ all distinct, then $\Delta^{m}$ is smooth at $D$ with tangent space equal to the subspace of $\Gamma\left(o_{C}(D) / o_{C}\right)$ :

$$
T_{D}\left(\Delta^{m}\right)=\text { Annihilator }\left[\Gamma\left(K_{C}((m-1) p-D) / K_{C}(-D)\right)\right]
$$

and

iii) The tangent space to $C_{d}^{1}$ at $D$ with $h^{0}\left(C, o_{C}(D)\right)=2$ is given as the annihilator of the image of the map

$$
\tilde{\mu}_{0}=r \circ \mu_{0}
$$

\footnotetext{
${ }^{3}$ In point of fact, the statement made in [7] is that $C_{d}^{1}$ intersects the cycle $x_{p_{1}} \cap \ldots \cap x_{p_{2 d-8-1}}$ transversely for $p_{1}, \ldots, p_{2 d-8-1}$ general points of $C$; but the argument applies equally in this case
} 
where

$$
\mu_{0}: H^{0}(C, o(D)) \otimes H^{0}\left(C, K_{C}(-D)\right) \rightarrow H^{0}\left(C, K_{C}\right)
$$

is multiplication, and

$$
r: H^{0}\left(C, K_{C}\right) \rightarrow H^{0}\left(C, K_{C} / K_{C}(-D)\right)
$$

is evaluation at $D$.

Statements i) and iii) are standard (cf. [2]), and ii) is elementary. Combining them, we see that a divisor $D$ on $C$, whose associated line bundle $L={ }_{0} C(D)$ satisfies the conditions of Theorem $\mathrm{B}$, is a transverse point of intersection of $C_{d}^{1}$ and $\Delta^{2 d-g}$ if and only if

$$
H^{0}\left(C, K_{C}(-2 D+(2 d-g-1) p)\right)=0 .
$$

To see that this in fact holds, let $D$ be such a divisor, and

$$
\pi: C \rightarrow \mathbf{P}^{1}
$$

be the corresponding map; let

$$
R_{\pi}=(2 d-g-1) p+q_{1}+\ldots+q_{3 g-1}
$$

be the ramification divisor of $\pi$. Let $\mathscr{H}$ be the versal deformation space for the map $\pi$, and let $\mathscr{H}^{\prime} \subset \mathscr{H}$ be the subvariety of $\mathscr{H}$ of maps with a $(2 d-g)$-fold branch point over $\pi(p)$. Then the tangent space to $\mathscr{H}$ at $\pi$ is given by

$$
T_{\pi}(\mathscr{H})=H^{0}(C, \eta)
$$

where $\eta$ is the normal sheaf of $\pi$, defined by the exact sequence

$$
0 \longrightarrow \theta_{C} \longrightarrow \pi^{*} \theta_{\mathbf{P}^{1}} \longrightarrow \eta \longrightarrow 0,
$$

and the differential of the map

$$
\phi: \mathscr{H} \rightarrow \mathscr{M}_{\mathrm{g}}
$$

is given by the coboundary map

$$
H^{0}(C, \eta) \rightarrow H^{1}\left(C, \theta_{C}\right)
$$

in this sequence. Finally, we may identify the tangent space to $\mathscr{H}^{\prime}$ at $\pi$ with the subspace of $T_{\pi}(\mathscr{H})=H^{0}(C, \eta)$ of sections of $\eta$ vanishing in a neighborhood of $p$, i.e., the sections of $\eta^{\prime}$ :

$$
0 \longrightarrow \theta_{C} \longrightarrow \pi^{*} \theta_{\mathbf{P}}(-(m-1) p) \longrightarrow \eta^{\prime} \longrightarrow 0 .
$$

Now, we observe that since $\pi$ is a point in a general fiber of $\left.\phi\right|_{\mathscr{H}}$, by Sard's theorem the differential $\phi_{*}$, restricted to $T_{\pi}(\mathscr{H})$, must be surjective. This means that the map $d$ below is surjective:

$$
H^{0}\left(\eta^{\prime}\right) \stackrel{d}{\longrightarrow} H^{1}\left(\theta_{C}\right) \longrightarrow H^{1}\left(\pi^{*} \theta_{\mathbf{P}^{1}}(-(m-1) P)\right) \longrightarrow 0 .
$$


But $\theta_{\mathbf{P}^{1}} \cong o_{\mathbf{P}^{1}}(2)$ and $\pi^{*} o_{\mathbf{P}^{1}}(1)=o_{C}(D)$, hence

as required.

$$
\begin{aligned}
(0) & =H^{1}\left(o_{C}(2 D-(m-1) P)\right) \\
& =H^{0}\left(K_{C}(-2 D+(m-1) P)\right)^{*},
\end{aligned}
$$

Using Theorems A and B, we can work out the intersection multiplicities in $\left(\operatorname{Int} \Delta_{i}\right) \cap \bar{D}_{k}$ :

Theorem 6. a) In the notation of Corollary $1, \Delta_{0}$ and $\bar{D}_{k}$ intersect generically transversely:

$$
\text { (Int } \left.\Delta_{0}\right) \cdot \overline{D_{k}}=\left\{\left(C^{\prime}, p_{1}, p_{2}\right) \mid \begin{array}{c}
\exists L \text { on } C^{\prime} \text { of degree } k \\
h^{0}(L) \geqq 2, h^{0}\left(L\left(-p_{1}-p_{2}\right)\right) \geqq 1
\end{array}\right\} \text { w. mult. one }
$$

b) In the notation of Corollary 3 , if $g_{1} \geqq 3$

$$
\begin{aligned}
\left(\text { Int } \Delta_{g_{1}}\right) \cdot \bar{D}_{k} & =\sum_{\frac{g_{1}+1}{2} \leqq k_{1} \leqq \min \left(k, g_{1}\right)} a\left(k_{1}+g_{2}-k+1, g_{2}\right) \cdot S_{k_{1}, 2 k_{1}-g_{1}, g_{1}} \times \mathscr{M}_{g_{2}, 1} \\
& +\sum_{\frac{g_{2}+1}{2} \leqq k_{2} \leqq \min \left(k, g_{2}\right)} a\left(k_{2}+g_{1}-k+1, g_{1}\right) \mathscr{M}_{g_{1}, 1} \times S_{k_{2}, 2 k_{2}-g_{2}, g_{2}} .
\end{aligned}
$$

This is still true for $g_{1}=1$ and 2 if the intersection is taken, not in $\overline{\mathscr{M}}_{g}$, but in the universal deformation space of a curve in Int $\Delta_{g_{1}}$ (n.b. Int $\Delta_{1}$ and Int $\Delta_{2}$ have divisors in the singular locus of $\overline{\mathscr{M}}_{g}$, so the intersection product is not welldefined).

Proof of a). In the set-theoretic intersection Int $\Delta_{0} \cap \bar{D}_{k}$, there is an open dense set consisting of those $C=C^{\prime} /\left(p_{1} \sim p_{2}\right)$ for which

0) $C^{\prime}$ has no automorphisms

i) there is a unique line bundle $L$ on $C^{\prime}$ of degree $k$ with $h^{0}(L) \geqq 2$, $h^{0}\left(L\left(-p_{1}-p_{2}\right)\right) \geqq 1$.

ii) for this $L, h^{0}(L)=2$ and $H^{0}(L)$ generates $L$

iii) if $\pi: C^{\prime} \rightarrow \mathbf{P}^{1}$ is the covering of degree $k$ defined by $H^{0}(L)$, then $\pi$ has only ordinary branch points $q_{i}$ and $\pi\left(q_{i}\right), \pi\left(p_{i}\right)$ are all distinct points of $\mathbf{P}^{1}$.

In fact, by Theorem $\mathrm{B}$, for almost all $C^{\prime}$ of genus $g-1=2 k-2$, there are $b(k, 2 k-2)$ pairs $(L, q)$ of line bundles $L$ such that $\operatorname{deg} L=k, h^{0}(L) \geqq 2$ and points $q \in C^{\prime}$ such that

$$
h^{0}(L(-2 q)) \geqq 1 .
$$

Each distinct $L_{k}$ defines a covering $\pi_{k}: C^{\prime} \rightarrow \mathbf{P}^{1}$, hence a curve

$$
\begin{aligned}
& \Gamma_{k} \subset C^{\prime} \times C^{\prime} \\
& \Gamma_{k}=\left\{(x, y) \mid \pi_{k} x=\pi_{k} y\right\} .
\end{aligned}
$$

Since almost all $\left(p_{1}, p_{2}\right) \in C^{\prime} \times C^{\prime}$ are on a unique curve $\Gamma_{k}$ if they are on any of them, this proves i), ii) and iii).

Now for such $C=C^{\prime} /\left(p_{1} \sim p_{2}\right),[C]$ is the image of a unique point of $\bar{H}_{k, b}$, namely the admissible cover $\pi: C^{\prime \prime} \rightarrow P$ of degree $k$ : 


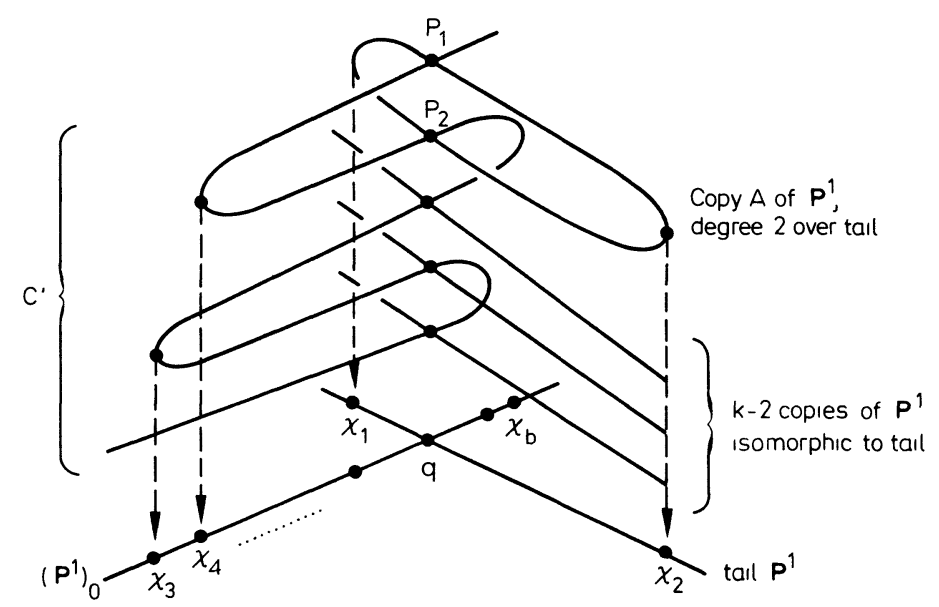

More precisely, if $\Sigma_{b}$ is the permutation group on $b$ letters, $\Sigma_{b}$ acts on $\bar{H}_{k, b}$ by permuting the labelling of the branch points $\left\{x_{i}\right\}$ and this covering is the unique point of $\bar{H}_{k, b} / \Sigma_{b}$ over [C]. Note that this admissible covering has an automorphism $\phi$ of order 2, however:

$\phi=\mathrm{id}$, on $C^{\prime}$

$\left.\phi\right|_{\text {tail } \mathbf{P}^{1}}=$ automorphism fixing $q$, interchanging the two branch points $x_{1}, x_{2}$ on the tail

$\left.\phi\right|_{\text {copy } A}=$ automorphism fixing $p_{1}, p_{2}$, interchanging the two ramified points.

However, $C^{\prime \prime}$ has no automorphisms over $P$ fixing all the points of ramification. Therefore, if $z$ is the point of $\bar{H}_{k, b}$ representing $\pi: C^{\prime \prime} \rightarrow P, z$ is a smooth point but if $\sigma \in \Sigma_{b}$ is the permutation interchanging $x_{1}, x_{2}$ and fixing the other branch points, it follows that $\sigma z=z$. In fact, $\sigma$ fixes the smooth divisor $\Delta \subset \bar{H}_{k, b}$ of all admissible coverings like $\pi: C^{\prime \prime} \rightarrow P$ where $P=\left(\mathbf{P}^{1}\right)_{0} \cup\left(\right.$ tail $\left.\mathbf{P}^{1}\right)$ but where $x_{3}, \ldots, x_{b}$ are allowed to vary in $\left(\mathbf{P}^{1}\right)_{0}$, and $C^{\prime}$ varies accordingly. There is a set $t_{1}, \ldots, t_{b-3}$ of local coordinates on $\bar{H}_{k, b}$ near $z$ such that at all points of $\pi^{-1}(q)$, the universal family

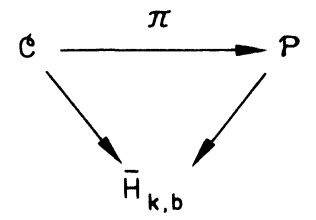

has local equations

$$
x y=t_{1},
$$

$t_{1}=0$ is the local equation of $\Delta$, and

$$
\begin{aligned}
\sigma^{*}\left(t_{1}\right) & =-t_{1} \\
\sigma^{*}\left(t_{i}\right) & =t_{i}, \quad 2 \leqq i \leqq b-3 .
\end{aligned}
$$


Then $t_{1}^{2}, t_{2}, \ldots, t_{b-3}$ are local coordinates on $\bar{H}_{k, b} /\{e, \sigma\}$ near the image of $z$.

Now consider the local analytic curve $\gamma: \Delta_{s} \rightarrow \bar{H}_{k, b} /\{e, \sigma\}$ given by $t_{1}^{2}=s$, $t_{2}=\ldots=t_{b-3}=0$. Consider the diagram:

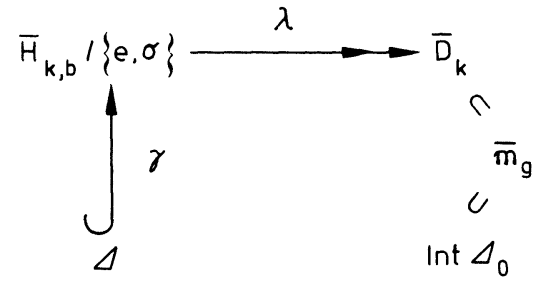

We shall check that the curve $\lambda \circ \gamma$ in $\overline{\mathscr{M}}_{\mathrm{g}}$ is transverse to Int $\Delta_{0}$ at the point [C]. This implies that the divisor $\lambda^{*}\left(\operatorname{Int} \Delta_{0}\right)$ on $\bar{H}_{k, n} /\{e, \sigma\}$ is $\Delta$ with multiplicity one, hence $\bar{D}_{k}$ and Int $\Delta_{0}$ meet transversely. To see that $\lambda \circ \gamma: \Delta \rightarrow \bar{M}_{g}$ is transverse to $\Delta_{0}$, consider the restriction of $\mathscr{C}$ to $t_{2}=\ldots=t_{b-3}=0$. It is a family of curves with only ordinary double points:

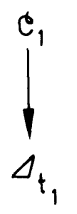

whose fibres are smooth if $t_{1} \neq 0$, and is

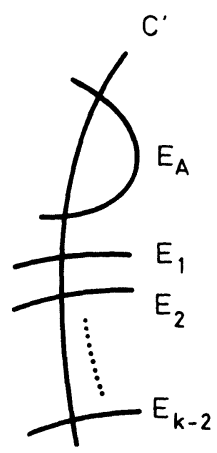

over $t_{1}=0$. Moreover, $\mathscr{C}_{1}$, as a surface, is smooth because its local equation is $x y=t_{1}$. It follows that on $\mathscr{C}_{1}$,

$$
\left(E_{A}^{2}\right)=-2,\left(E_{1}^{2}\right)=\ldots=\left(E_{k-2}^{2}\right)=-1 .
$$

Blowing down the $E$ 's, we get a family of stable curves

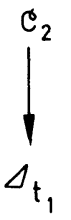


with the same curves when $t_{1} \neq 0$, and with fibre $C$ over $t_{1}=0$. Since $E_{A}$ blows down to an ordinary surface double point, $\mathscr{C}_{2}$ has equation $x y=t_{1}^{2}$ at the image of $E_{A}$. But $\mathscr{C}_{2} / \Delta_{t_{1}}$ is just the pull-back to $\Delta_{t_{1}}$ of the family of curves over $\Delta_{s}$ given by the morphism $\lambda \circ \gamma: \Delta_{s} \rightarrow \overline{\mathscr{M}}_{\mathrm{g}}$. Thus it is induced by a family

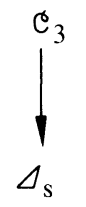

where $\mathscr{C}_{3}$ has local equation $x \cdot y=s$ at the image of $E_{A}$ and is again smooth. This means that $\mathscr{C}_{3}$ restricts to the universal deformation of the singular point of $C$, hence is a curve on $\overline{\mathscr{M}}_{g}$ transverse to the locus $\Delta_{0}$ of singular curves.

Proof of b). The situation is similar with the two sums, so let's take a sufficiently general point $[C] \in S_{k_{1}, 2 k_{1}-g_{1}, g_{1}} \times \mathscr{M}_{g_{2}, 1}$ and compute the intersection multiplicity here. Now $C=C_{1} \cup C_{2}, C_{1}$ of genus $g_{1}, C_{2}$ of genus $g_{2}$, $C_{1} \cap C_{2}=\{p\}$. Since by Theorem B for almost all $C_{1}$ of genus $g_{1}$, there are $b\left(k_{1}, g_{1}\right)$ pairs $\left(L_{1}, p\right)$ such that $\operatorname{deg} L_{1}=k_{1}, h^{0}\left(L_{1}\right) \geqq 2, h^{0}\left(L_{1}\left(-\left(2 k_{1}-g_{1}\right) p\right)\right) \geqq 1$, and since for almost all $C_{1}$, the points $p$ are distinct, it follows that for almost all $\left(C_{1}, p\right) \in S_{k_{1}, 2 k_{1}-g_{1}, g_{1}}$, there is a unique $L_{1}$ of degree $k_{1}$ with $h^{0}\left(L_{1}\right) \geqq 2$, $h^{0}\left(L_{1}\left(-\left(2 k_{1}-g_{1}\right) p\right)\right) \geqq 1$. Moreover, for this $L_{1}, h^{0}\left(L_{1}\right)=2$ and $H^{0}\left(L_{1}\right)$ defines a covering

$$
\pi_{1}: C_{1} \rightarrow\left(\mathbf{P}^{1}\right)_{1}
$$

with general branching except for a $\left(2 k_{1}-g_{1}\right)$-fold branch point at $p$. As for $\left(C_{2}, p\right)$, this is a general point of $\mathscr{M}_{g_{2}, 1}$, hence by Theorem $\mathrm{A}$ for all $k_{2}$ it has exactly $a\left(k_{2}, g_{2}\right)$ line bundles $L_{2}$ of degree $k_{2}$ with

$$
h^{0}\left(L_{2}\right) \geqq 2, \quad h^{0}\left(L_{2}\left(-\left(2 k_{2}-g_{2}-1\right) p\right)\right) \geqq 1 .
$$

Moreover, for all of these, $h^{0}\left(L_{2}\right)=2$ and $H^{0}\left(L_{2}\right)$ defines

$$
\pi_{2}: C_{2} \rightarrow\left(\mathbf{P}^{1}\right)_{2}
$$

with general branching except for a $\left(2 k_{2}-g_{2}\right)$-fold branch point at $p$. In particular, there are no line bundles $L$ at all of degree $k_{2}$ with $h^{0}\left(L_{2}\right) \geqq 2$, $h^{0}\left(L_{2}\left(-\left(2 k-g_{2}\right) p\right)\right) \geqq 1$. A little reflection shows that the only ways to lift $C$ to a point of $\bar{H}_{k, b}$ are to use the admissible coverings defined by $L_{1}$ on $C_{1}$ of degree $k_{1}$ and $L_{2}$ on $C_{2}$ of degree $k_{2}$ where both have $\ell$-fold branching at $p$ and $\ell=2 k_{1}-g_{1}=2 k_{2}-g_{2}-1$ :

Example. $\ell=2$,

$k_{1}=4$,

$k_{2}=3$,

$k=5$. 


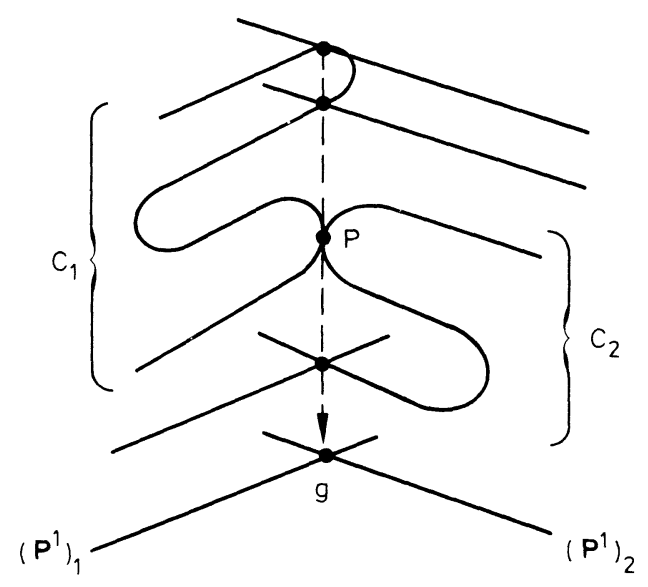

Here the degree of $\pi$ is $k$ and over $\left(\mathbf{P}^{1}\right)_{1}$ we have $C_{1}$ of degree $k_{1}$ and $k-k_{1}$ copies of $\mathbf{P}^{1}$ mapping isomorphically to $\left(\mathbf{P}^{1}\right)_{1}$; and over $\left(\mathbf{P}^{1}\right)_{2}$, we have $C_{2}$ of degree $k_{2}$ and $k-k_{2}$ copies of $\mathbf{P}^{1}$ mapping isomorphically to $\left(\mathbf{P}^{1}\right)_{2}$.

In order to do this, we must choose $k_{2}$ by the equation:

i.e.,

$$
2 k_{2}-g_{2}-1=2 k_{1}-g_{1} \text {, }
$$

$$
\begin{aligned}
k_{2} & =k_{1}+\frac{g_{2}+1-g_{1}}{2} \\
& =k_{1}+g_{2}+\frac{1-g_{1}-g_{2}}{2} \\
& =k_{1}+g_{2}-k+1 .
\end{aligned}
$$

Now, whereas $L_{1}$ is unique, there are $a\left(k_{2}, g_{2}\right)$ choices for $L_{2}$, so this gives exactly $a\left(k_{2}, g_{2}\right)$ points of $\bar{H}_{k, b} / \Sigma_{b}$ over $[C] \in \bar{D}_{k}$. Thus $\bar{D}_{k}$ is a divisor with $a\left(k_{2}, g_{2}\right)$ branches at [C], and what the theorem says is that each of these branches meets Int $\Delta_{g_{1}}$ transversely. Assuming to begin with that $g_{1} \geqq 3$, the argument is similar to case (a) except simpler because the covering $\pi: C^{\prime \prime} \rightarrow P$ $=\left(\mathbf{P}^{1}\right)_{1} \cup\left(\mathbf{P}^{1}\right)_{2}$ has no automorphisms. Thus $\bar{H}_{k, b}$ is smooth at the point $z$ representing this covering and $z$ is not fixed by any $\sigma \in \Sigma_{b}$. If we embed $\pi: C^{\prime \prime} \rightarrow P$ in a one-dimensional family of admissible coverings, we get

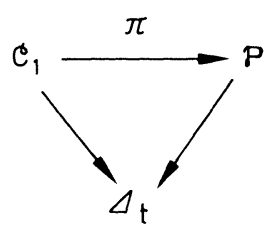

where locally near $p$

$$
\begin{array}{ll}
\mathscr{C}_{1} & \text { is } \quad x \cdot y=t, \\
\mathscr{P} & \text { is } \quad u \cdot v=t^{\ell}, \\
\pi & \text { is } \quad u=x^{\ell}, v=y^{\ell} .
\end{array}
$$


Thus $\mathscr{C}_{1}$ is a smooth surface. All the extra rational curves in the fibre of $\mathscr{C}_{1}$ over $t=0$ are curves $E$ with $\left(E^{2}\right)=-1$. Blowing these down, we get a family of stable curves

$$
\mathscr{C}_{2} \rightarrow \Delta_{t}
$$

where $\mathscr{C}_{2}$ is still smooth and the fibre over $t=0$ is $C$. Therefore this family defines a curve in $\bar{M}_{g}$ transverse to $\Delta_{g_{1}}$ at $[C]$.

Now in case $g_{1}=1$ or 2 , there is the extra complication that $C$ may have automorphisms. Rather than working on the coarse moduli space, it is more convenient to use the same argument to analyze the intersection of the 2 divisors Int $\Delta_{g_{1}}$ and $\bar{D}_{k}$ in the universal deformation $\Delta^{3 g-3}$ of $C$. The universal deformation of $\pi: C^{\prime \prime} \rightarrow P$ gives us a family

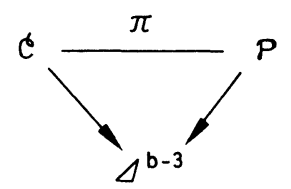

plus a uniformization

$$
\Delta^{b-3} \rightarrow \bar{D}_{k} \subset \Delta^{3 g-3}
$$

of the branch of $\bar{D}_{k}$ near $[C]$ defined by $\pi: C^{\prime \prime} \rightarrow P$. Restricting this deformation to a one-dimensional family as above, we get a curve on $\Delta^{b-3}$ mapped to a curve in $\Delta^{3 g-3}$ transverse to the boundary component. Hence these divisors meet transversely in $\Delta^{3 g-3}$.

\section{§6. The Class of the Divisor $\bar{D}_{k}$, II}

In this section we finally compute the divisor class of $\bar{D}_{k}$ in $\operatorname{Pic}\left(\overline{\mathscr{M}}_{\text {g, reg }}\right)$ as a combination of $\lambda, \delta_{0}, \ldots, \delta_{k-1}$. We start with the relation

$$
\bar{D}_{k} \equiv a \lambda-b_{0} \delta_{0}-b_{1} \delta_{1}-\ldots-b_{k-1} \delta_{k-1}
$$

proved in $\S 3$, but with unknown constants $a, b_{i}$, and we determine the constants by restricting this relation to suitable curves in $\overline{\mathscr{M}}_{\mathrm{g}}$.

To describe the general method, suppose $D$ is any divisor on $\overline{\mathscr{M}}_{g}$, i.e., a combination of codimension 1 subvarieties, but not necessarily a Cartier divisor (=one with a single local equation $f \in \mathbf{C}\left(\mathscr{M}_{g}\right)^{*}$ everywhere). Assume

$$
D \equiv a \lambda-b_{0} \delta_{0}-\ldots-b_{k-1} \delta_{k-1}
$$

in $\operatorname{Pic}\left(\overline{\mathscr{M}}_{g \text {,reg }}\right)$. Let $S$ be a smooth projective curve, let

$$
\pi: \mathscr{C} \rightarrow S
$$

be a family of stable curves of genus $g$, and let

$$
\gamma: S \rightarrow \overline{\mathscr{M}}_{\mathrm{g}}
$$

be the induced morphism. Then first of all if $\gamma(S) \notin \operatorname{Supp}(D)$ we can define the divisor $\gamma^{*} D$ in a canonical way (even though $\gamma(S)$ may meet $\operatorname{Sing}\left(\overline{\mathscr{M}}_{\mathrm{g}}\right) \cap \operatorname{Supp} D$ ). From general principles, we can apply the homomorphism: 


$$
\operatorname{Pic}\left(\overline{\mathscr{M}}_{g, \text { reg }}\right) \rightarrow \operatorname{Pic}_{\text {fun }}\left(\overline{\mathscr{M}}_{\mathrm{g}}\right)
$$

to $\sigma_{\mathcal{M}_{g, \text { reg }}}(D)$ and "evaluate" the image on the family $\pi: \mathscr{C} \rightarrow S$, to get a line bundle on $S$. But even more, for every $s \in S$, let

$$
\pi_{s}: \mathscr{C}_{s} \rightarrow \Delta^{3 g-3}
$$

be the universal deformation of the curve $C_{s}$ which is the fibre of $\pi$ over $s$. Then the morphism $\gamma$ factors:

$$
\left(\begin{array}{c}
\text { neigh of } \\
s \in S
\end{array}\right) \underset{\gamma_{1}}{\longrightarrow} \Delta^{3 g-3} \underset{\gamma_{2}}{\longrightarrow} \overline{\mathscr{M}}_{g} .
$$

$\gamma_{2}^{*}(D)$ is a divisor on $\Delta^{3 g-3}$, hence a Cartier divisor (as $\Delta^{3 g-3}$ is smooth), and we then pull it back by $\gamma_{1}$ as Cartier divisor:

$$
\gamma^{*}(D) \underset{\operatorname{def}}{=} \gamma_{1}^{*}\left(\gamma_{2}^{*} D\right)
$$

On the other hand, $\gamma^{*}(\lambda)$ is defined directly as $c_{1}$ of the line bundle $\Lambda^{g} \pi_{*} \omega_{\mathscr{C} / S}$, and $\gamma^{*} \delta_{i}$ are defined directly too as in Knudsen [10]. That is to say, if $\gamma(S) \phi$ $\Delta_{i}, \gamma^{*} \delta_{i}$ is represented by the Cartier divisor $\gamma^{*} \Delta_{i}(i \neq 1)$ or $\frac{1}{2} \gamma^{*} \Delta_{1}(i=1)$. And if $\gamma(S) \subset \Delta_{i}$ and the curves $C_{s}$ have exactly one double point $x_{s}$ of type $i$, then $\gamma^{*} \delta_{i}$ is $c_{1}$ of the line boundle:

$$
s \mapsto \Lambda^{2}\left(\Omega_{C_{s}}^{1} \otimes \mathbf{k}\left(x_{s}\right)\right)^{*} .
$$

For our first family, choose general curves $C_{1}, C_{2}$ of genera $\alpha \leqq k-1$ and $2 k-1-\alpha$ respectively. Choose $p \in C_{2}$ a general point and let $S_{2}=C_{1} \times\{p\} \subset C_{1}$ $\times C_{2}$; denote by $S_{1}$ the diagonal $\Delta \subset C_{1} \times C_{1}$; and let

$$
\pi: T \rightarrow C_{1}
$$

be the curve over $C_{1}$ obtained by identifying $S_{1}$ and $S_{2}$ in $C_{1} \times C_{1} \cup C_{1} \times C_{2}$ over $C_{2}$. This is, the family whose fiber over $q \in C_{1}$ is the reducible curve obtained by identifying $q \in C_{1}$ with $p \in C_{2}$.

The degrees of the divisors $\lambda$ and $\delta_{i}$ on $T$ are readily calculated: first of all, we see that $\pi_{*} \omega_{T / C_{1}}$ is the trivial bundle $\left(H^{0}\left(C_{1}, \omega_{C_{1}}\right) \oplus H^{0}\left(C_{2}, \omega_{C_{2}}\right)\right) \otimes{ }{ }_{C_{1}}$, so that $\operatorname{deg} \lambda=0$. Clearly, $\operatorname{deg} \delta_{i}=0$ on $T$ for $i \neq \alpha$; and since the normal space to $\Delta_{\alpha}$ in $\bar{M}_{2 k-1}$ at the point $T_{q}$ is the tensor product $T_{q}\left(C_{1}\right) \otimes T_{p}\left(C_{2}\right)$, we have

$$
\begin{aligned}
\operatorname{deg} \delta_{\alpha} & =\operatorname{deg}\left(N_{\Delta_{\alpha} / \mathscr{M}} \otimes \sigma_{T}\right) \\
& =\operatorname{deg}\left(N_{S_{1} / C_{1} \times C_{2}} \otimes N_{S_{2} / C_{1} \times C_{2}}\right) \\
& =2-2 \alpha .
\end{aligned}
$$

It remains to calculate the degree of the divisor $\bar{D}_{k}$ on $T$. By $\S 4$, Theorem 5 , Corollary 3 , set-theoretically

$$
\bar{D}_{k} \cap C_{1}=\left\{\begin{array}{l|l}
q \in C_{1} & \begin{array}{l}
\text { For some } i, 0 \leqq i \leqq \frac{\alpha-1}{2} \\
\exists \text { line bundle } L \text { on } C_{1} \text { of degree } \alpha-i \\
\text { with } \left.h^{0}(L) \geqq 2, h^{0}(L(-(\alpha-2)) q)\right) \geqq 1
\end{array}
\end{array}\right\}
$$


which by Theorem B consists in $b(\alpha-i, \alpha)$ points. But by Theorem 6 , the multiplicity of each point in $a(k-i, 2 k-1-\alpha)$. (Note that for generic $C_{1}$, the divisor $S_{\alpha-i, \alpha-2 i, \alpha}$ on $\mathscr{M}_{\alpha, 1}$ and the curve $\left\{\left(C_{1}, q\right) \mid q \in C_{1}\right\}$ on $\mathscr{M}_{\alpha, 1}$ must meet transversely.) Therefore

$$
\begin{aligned}
\operatorname{deg}_{C_{1}} \bar{D}_{k} & =\sum_{i=0}^{\frac{\alpha-1}{2}} b(\alpha-i, \alpha) a(k-i, 2 k-1-\alpha) \\
& =\sum_{i=0}^{\alpha / 2} \frac{\alpha !(2 k-1-\alpha) !}{(\alpha-i) ! i !(k-i) !(k-\alpha+i) !}(\alpha-2 i-1)(\alpha-2 i)^{2}(\alpha-2 i+1) \\
& =\frac{1}{2} \sum_{i=0}^{\alpha} \frac{\alpha !(2 k-1-\alpha) !}{(\alpha-i) ! i !(k-i) !(k-\alpha+i) !}(\alpha-2 i-1)(\alpha-2 i)^{2}(\alpha-2 i+1) .
\end{aligned}
$$

(The last equality coming from the fact that the sum is unaltered under the substitution $i \rightsquigarrow \alpha-i$.) Now, writing

$$
\begin{aligned}
(\alpha-2 i-1)(\alpha-2 i)^{2}(\alpha-2 i+1) & \\
= & {[(k-i)-(k-\alpha+i)] \times[(\alpha-i)(\alpha-i-1)(\alpha-i-2)-3(\alpha-i)(\alpha-i-1) i} \\
& +3(\alpha-i) i(i-1)-i(i-1)(i-2)+3(\alpha-i)(\alpha-i-1)-3 i(i-1)],
\end{aligned}
$$

this sum becomes

$$
\begin{aligned}
& \frac{\alpha(\alpha-1)(\alpha-2)}{2} \sum_{i=0}^{\alpha}\left[\left(\begin{array}{c}
\alpha-3 \\
i
\end{array}\right)\left(\begin{array}{c}
2 k-1-\alpha \\
k-i-1
\end{array}\right)-3\left(\begin{array}{c}
\alpha-3 \\
i-1
\end{array}\right)\left(\begin{array}{c}
2 k-1-\alpha \\
k-i-1
\end{array}\right)\right. \\
& +3\left(\begin{array}{c}
\alpha-3 \\
i-2
\end{array}\right)\left(\begin{array}{c}
2 k-1-\alpha \\
k-i-1
\end{array}\right)-\left(\begin{array}{c}
\alpha-3 \\
i-3
\end{array}\right)\left(\begin{array}{c}
2 k-1-\alpha \\
k-i-1
\end{array}\right) \\
& -\left(\begin{array}{c}
\alpha-3 \\
i
\end{array}\right)\left(\begin{array}{c}
2 k-1-\alpha \\
k-i
\end{array}\right)+3\left(\begin{array}{c}
\alpha-3 \\
i-1
\end{array}\right)\left(\begin{array}{c}
2 k-1-\alpha \\
k-i
\end{array}\right) \\
& \left.-3\left(\begin{array}{c}
\alpha-3 \\
i-2
\end{array}\right)\left(\begin{array}{c}
2 k-1-\alpha \\
k-i
\end{array}\right)+\left(\begin{array}{c}
\alpha-3 \\
i-3
\end{array}\right)\left(\begin{array}{c}
2 k-1-\alpha \\
k-i
\end{array}\right)\right] \\
& +\frac{\alpha(\alpha-1)}{2} \sum_{i=0}^{\alpha}\left[3\left(\begin{array}{c}
\alpha-2 \\
i
\end{array}\right)\left(\begin{array}{c}
2 k-1-\alpha \\
k-i-1
\end{array}\right)-3\left(\begin{array}{c}
\alpha-2 \\
i-2
\end{array}\right)\left(\begin{array}{c}
2 k-1-\alpha \\
k-i-1
\end{array}\right)\right. \\
& \left.-3\left(\begin{array}{c}
\alpha-2 \\
i
\end{array}\right)\left(\begin{array}{c}
2 k-1-\alpha \\
k-i
\end{array}\right)+3\left(\begin{array}{c}
\alpha-2 \\
i-2
\end{array}\right)\left(\begin{array}{c}
2 k-1-\alpha \\
k-i
\end{array}\right)\right] \\
& =\frac{\alpha(\alpha-1)(\alpha-2)}{2}\left[-6\left(\begin{array}{c}
2 k-4 \\
k-2
\end{array}\right)+8\left(\begin{array}{c}
2 k-4 \\
k-3
\end{array}\right)-2\left(\begin{array}{c}
2 k-4 \\
k-4
\end{array}\right)\right] \\
& +\frac{\alpha(\alpha-1)}{2}\left[3\left(\begin{array}{c}
2 k-3 \\
k-2
\end{array}\right)-3\left(\begin{array}{c}
2 k-3 \\
k-3
\end{array}\right)\right] \\
& =-6 \alpha(\alpha-1)(\alpha-2) \cdot \frac{(2 k-4) !}{k !(k-2) !}+6 \alpha(\alpha-1) \frac{(2 k-3) !}{k !(k-2) !} \\
& =6 \alpha(\alpha-1)(2 k-1-\alpha) \frac{(2 k-4) !}{k !(k-2) !} \text {. }
\end{aligned}
$$


We conclude, then, that

$$
\begin{aligned}
b_{\alpha} & =\frac{\operatorname{deg} D_{k}}{2 \alpha-2} \\
& =\frac{3 \alpha(2 k-1-\alpha)}{k} \cdot \frac{(2 k-4) !}{(k-1) !(k-2) !}
\end{aligned}
$$

for $\alpha \geqq 2$.

Note that if we choose to vary the point $p \in C_{2}$ and fix $q$ to be a general point of $C_{1}$, the resulting family $\pi: T \rightarrow C_{2}$ has intersection numbers

$$
\begin{aligned}
\operatorname{deg} \lambda & =0, \\
\operatorname{deg} \delta_{i} & =0, \quad i \neq \alpha, \\
\operatorname{deg} \delta_{\alpha} & =2-2(2 k-1-\alpha), \\
& =-2(2 k-2-\alpha)
\end{aligned}
$$

and, as before,

$$
\begin{aligned}
\operatorname{deg} D_{k} & =\sum_{i=0}^{\frac{\alpha+1}{2}} a(\alpha-i+1, \alpha) \cdot b(k-i, 2 k-1-\alpha) \\
& =\sum_{i=0}^{\frac{\alpha+1}{2}} \frac{\alpha !(2 k-1-\alpha) !}{(\alpha-i+1) ! i !(k-i) !(k+i-\alpha-1) !}(\alpha-2 i)(\alpha-2 i+1)^{2}(\alpha-2 i+2) .
\end{aligned}
$$

Setting $\beta=\alpha+1$, this is

$$
\begin{aligned}
& \sum_{i=0}^{\beta / 2} \frac{(\beta-1) !(2 k-\beta) !}{(\beta-i) ! i !(k-i) !(k+i-\beta) !}(\beta-2 i-1)(\beta-2 i)^{2}(\beta-2 i+1) \\
& \quad=\frac{2 k-\beta}{\beta} \cdot 6 \beta(\beta-1)(2 k-\beta-1) \frac{(2 k-4) !}{k !(k-2) !} \\
& \quad=6 \alpha(2 k-\alpha-1)(2 k-\alpha-2) \frac{(2 k-4) !}{k !(k-2) !}
\end{aligned}
$$

As before, then, we conclude that

$$
b_{\alpha}=\frac{\operatorname{deg} D_{k}}{2(2 k-2-\alpha)}=\frac{3 \alpha(2 k-\alpha-1)}{k} \frac{(2 k-4) !}{(k-1) !(k-2) !}
$$
i.e.,

$$
b_{1}=\frac{6(k-1)}{k} \cdot \frac{(2 k-4) !}{(k-1) !(k-2) !} \text {. }
$$

Our second family of curves lies entirely in $\Delta_{1}$, consisting of a fixed curve $C_{2}$ of genus $g-1$, plus a variable elliptic curve $E$ attached at a constant point of $C_{2}$. To construct it, let $\pi_{1}: X \rightarrow B$ be a map from a smooth surface $X$ to a curve $B$, whose fibers are all stable curves of genus 1 ; let $S_{1} \subset X$ be a section of the map. On the other hand, let $C_{2}$ be a general curve of genus $g-1, p \in C_{2}$ a general point, and $S_{2}=B \times\{p\} \subset B \times C_{2}$. Finally, we let

$$
\pi: U \rightarrow B
$$

be the curve over $B$ obtained by identifying $S_{1} \subset X$ and $S_{2} \subset B \times C_{2}$. 
To compute the degrees of the various divisors $\lambda, \delta_{\alpha}, D_{k}$ on $U$, note that if we set

$$
d=-\operatorname{deg} N_{S_{1} / X}
$$

then the degree of the $j$-function associated to $\pi_{1}$ is just $12 d$; accordingly

and

$$
\begin{aligned}
\operatorname{deg} \delta_{0} & =12 d, \\
\operatorname{deg} \lambda & =\operatorname{deg} \pi_{*} \omega_{U / \boldsymbol{B}} \\
& =\operatorname{deg}\left(\pi_{1}\right)_{*} \omega_{X / \boldsymbol{B}} \\
& =d,
\end{aligned}
$$

$$
\begin{aligned}
\operatorname{deg} \delta_{1} & =\operatorname{deg}\left(N_{S_{1} / X} \otimes N_{S_{2 / B} \times C_{2}}\right) \\
& =\operatorname{deg} N_{S_{1} / X} \\
& =-d .
\end{aligned}
$$

Of course, $\operatorname{deg} \delta_{\alpha}=0$ for $\alpha \geqq 2$. On the other hand, we see from Corollary $4, \S 4$, that $U$ is disjoint from $\bar{D}_{k}$, since $C_{2}$, being general, possesses no line bundles $L$ of degree $k-1$ with $h^{0}(L)=2$ and only finitely many of degree $k$; and $p$, being a general point of $C_{2}$, will not be a branch point of any of the associated coverings $\pi: C_{2} \rightarrow \mathbf{P}^{1}$. We conclude, then, immediately

i.e.,

$$
a \cdot \operatorname{deg} \lambda-b_{0} \operatorname{deg} \delta_{0}-b_{1} \operatorname{deg} \delta_{1}=0,
$$

$$
a-12 b_{0}+b_{1}=0 .
$$

For our last family, take $C$ a general curve of genus $2 k-2, p \in C$ a general point. Let $S_{1}$ and $S_{2}$ be the proper transforms of the diagonal $\Delta$ and the crosssection $C \times\{p\}$ in the blow-up $\widetilde{C \times C}$ of $C \times C$ at the point $(p, p) . S_{1}$ and $S_{2}$ being disjoint, we may identify them in $\widetilde{C \times C}$ over $C$ to obtain a family

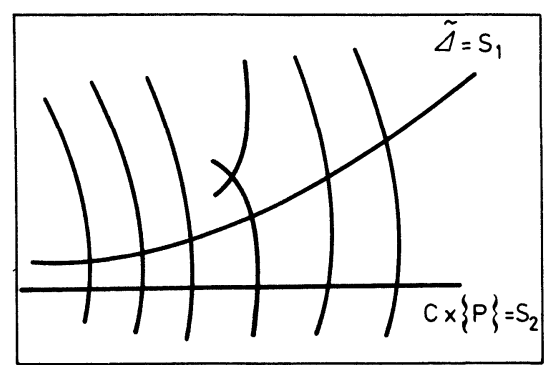

$\widetilde{\mathrm{C} \times \mathrm{C}}$

$W \stackrel{\pi}{\longrightarrow} C$ of stable curves of genus $g$ over $C$.

For this family, we have clearly

$$
\begin{aligned}
& \operatorname{deg} \delta_{1}=1, \\
& \operatorname{deg} \delta_{\alpha}=0, \quad \alpha>1
\end{aligned}
$$


and

$$
\begin{aligned}
\operatorname{deg} \delta_{0} & =\operatorname{deg}\left(N_{S_{1} / \widetilde{C \times C}} \otimes N_{S_{2} / \widetilde{C \times C}}\right) \\
& =(2-2(2 k-2)-1)-1 \\
& =4-4 k .
\end{aligned}
$$

We also have the sequence

$$
0 \rightarrow H^{0}\left(C, \omega_{C}\right) \otimes o_{C} \rightarrow \pi_{*} \omega_{W / C} \rightarrow o_{C} \rightarrow 0
$$

where the right-hand map is given by taking the residue at $S_{1}$, from which we conclude that

$$
\operatorname{deg} \lambda=c_{1} \pi_{*} \omega_{W / C}=0 .
$$

Finally, since $C$ and $p \in C$ are general, $C$ will possess exactly $\frac{(2 k-2) !}{(k-1) !(k) !}$ line bundles $L$ of degree $k$ with $h^{0}(L)=2$, each of which will have a unique section zero at $p$; and none of these sections will have a multiple zero. There are thus a total of $\frac{(2 k-2) !}{(k-1) !(k) !}(k-1)$ points $q \in C$ such that $h^{0}(L(-p-q))=1$ for one of these $L$ 's. By Theorem $6 \mathrm{a}$, these points occur with multiplicity one in the divisor induced by $\bar{D}_{k}$ on $C$, i.e.,

$$
\operatorname{deg}_{C} \bar{D}_{k}=\frac{(2 k-2) !}{k !(k-2) !}
$$

and we conclude that

$$
4(k-1) b_{0}-b_{1}=\frac{(2 k-2) !}{k !(k-2) !} .
$$

Since from (4) we have

$$
b_{1}=\frac{6(k-1)}{k} \frac{(2 k-4) !}{(k-1) !(k-2) !} .
$$

this yields

$$
\begin{aligned}
b_{0} & =\frac{1}{4(k-1)}\left(\frac{(2 k-2) !}{k !(k-2) !}+\frac{6(k-1)}{k} \frac{(2 k-4) !}{(k-1) !(k-2) !}\right) \\
& =\frac{1}{4} \cdot \frac{(2 k-4) !}{k !(k-2) !}(2(2 k-3)+6) \\
& =\frac{(2 k-4) !}{(k-1) !(k-2) !}
\end{aligned}
$$

and applying the relation (5), we have in turn

$$
\begin{aligned}
a & =12 b_{0}-b_{1} \\
& =\left(12-\frac{6(k-1)}{k} \frac{(2 k-4) !}{(k-1) !(k-2) !}\right) \\
& =\left(6+\frac{6}{k}\right) \cdot \frac{(2 k-4) !}{(k-1) !(k-2) !} .
\end{aligned}
$$


All in all, then, the coefficients appearing in the expression (1) are

where

$$
\begin{aligned}
a & =6(k+1) c, \\
b_{0} & =k c, \quad b_{\alpha}=3 \alpha(2 k-\alpha-1) c
\end{aligned}
$$

$$
c=\frac{(2 k-4) !}{k !(k-2) !} .
$$

\section{References}

1. Anderson, G.: Theta functions and holomorphic differential forms on compact quotients of bounded symmetric domains. Ph.D. Thesis, Princeton, 1980

2. Arbarello, E., Cornalba, M., Griffiths, P., Harris, J.: Topics in the theory of algebraic curves. In Princeton Univ. Press (1982 in press)

3. Brylinski, J.-L.: Propriétés de ramification à l'infini du groupe modulaire de Teichmüller. Ann. Ec. Norm. Sup., 12, 295 (1979)

4. Freitag, E.: Der Körper der Siegelschen Modulfunktionen. Abh. Math. Sem. Univ. Hamburg,

5. Freitag, E.: Die Kodairadimension von Körpern automorpher Funktionen. Crelle, 296, 162 (1977)

6. Fulton, W.: Hurwitz schemes and the irreducibility of moduli of algebraic curves, Annals of Math., 90, 542 (1969)

7. Griffiths, P., Harris, J.: On the variety of special linear systems on a general algebraic curve. Duke Math. J., 47, 233 (1980)

8. Grothendieck, A., Murre, J.P.: The tame fundamental group of a formal neighborhood of a divisor with normal crossings on a scheme. Lecture Notes Berlin-Heidelberg-New York: Springer Vol. 208. 1971

9. Kleiman, S., Laksov, D.: Another proof of the existence of special divisors. Acta Math., 132, 163 (1974)

10. Knudsen, F.: The projectivity of the moduli space of stable curves. Math. Scand. (in press 1982)

11. MacDonald, I.G.: Symmetric products of an algebraic curve. Topology, 1, 319 (1962)

12. Mumford, D.: Hirzebruch's Proportionality Principle in the non-compact case. Inv. Math., 42, 239 (1977)

13. Mumford, D.: Stability of projective varieties. L'Ens. Math., 23, 39 (1977)

14. Murre, J.P.: An introduction to Grothendieck's of the fundamental group, Tata Institute Lecture Notes; Bombay (1967)

15. Reid, M.: Canonical 3-folds. Les Journées de Géometrie Algébrique d'Angers, 1979, A. Beauville, ed.

16. Stillman, M.: PhD Thesis, Harvard, 1983

17. Tai, Y.-S.: Pluri-canonical differentials on the Siegel modular variety. Invent. Math. (in press 1982)

Oblatum 3-XI-1981

\section{Added in Proof}

Since this article was written, 2 improvements have been made in Theorem 3. John Harer has proven that $\operatorname{Pic}\left(M_{g}^{0}\right)$ is infinite cyclic, hence any divisor $D$ on $M_{g}^{0}$ satisfies

$$
[D] \equiv a \cdot \lambda, \quad a \in \mathbf{Q} \text {. }
$$

Secondly George Kempf has been able to carry through the calculation of the $a_{k}$ of Theorem 3 via Porteous' formula, confirming that

$$
a_{k}=6(2 k-4) !(k+1) / k !(k-2) !
$$

